\title{
Clickable Bambusurils to Access Multivalent Architectures
}

Marine Lafosse, Elise Cartier, Kathleen Solmont, Julie Rivollier, Djamille Azazna, Pierre Thuéry, Yves Boulard, Amandine Gontier, Jean-Baptiste Charbonnier, Bertrand Kuhnast, and Marie-Pierre Heck*

Université Paris-Saclay, CEA, Service de Chimie Bio-organique et de Marquage, 91191 Gif-sur-Yvette, France;

\section{Supporting Information}

Table of Contents

General Methods and equipment S2

ITC instrument and procedure S3

Synthetic procedures and characterization data S3

2,4-dipropargylglycoluril 1 S3

Octapropargylbambus[4]uril 2 S4

Tetrabutylammonium bromide @Dodecapropargylbambus[6]uril 3 S4

Tetrabutylammonium iodide @Dodecapropargylbambus[6]uril 4 S5

Tetrabutylammonium chloride @Dodecapropargylbambus[6]uril 5 S5-S6

Dodecapropargylbambus[6]uril 6 S6

Octakis (1-((4-ethoxy-4-oxobutyl)-1H-1,2,3-triazol-4-yl)methyl) bambus[4]uril 8a S6-S7

Octakis (1-(1- $\beta$-tetraacetylated glucose)-1H-1,2,3-triazol-4-yl)methyl) bambus[4]uril 8b S7

Octakis(1-(2-ethyl-2,3,4,6-tetra-O-acetyl- $\beta$-D-glucopyranoside)-1H-1,2,3-triazol-4-yl)methyl) bambus[4]uril 8c

Sodium bromide @Dodecakis 1-((4-ethoxy-4-oxobutyl)-1H-1,2,3-triazol-4-yl)methyl)

bambus[6]uril 9a

Sodium iodide @Dodecakis 1-((4-ethoxy-4-oxobutyl)-1H-1,2,3-triazol-4-yl)methyl)

bambus[6]uril 10a

Dodecakis 1-((4-ethoxy-4-oxobutyl)-1H-1,2,3-triazol-4-yl)methyl) bambus[6]uril 11a

Sodium bromide @Dodecakis (1-(2-ethyl-2,3,4,6-tetra-O-acetyl- $\beta$-D-glucopyranoside)

-1H-1,2,3-triazol4-yl)methyl) bambus[6]uril 9c

Dodecakis (1-(1- $\beta$-tetraacetylated glucose)-1H-1,2,3-triazol-4-yl)methyl)bambus[6]uril 11b

Dodecakis (1-(2-ethyl-2,3,4,6-tetra-O-acetyl- $\beta$-D-glucopyranoside)-1H-1,2,3-triazol4-yl) methyl) bambus[6]uril 11c

Dodecakis (1-(4 butanoic acid)-1H-1,2,3-triazol-4-yl)methyl) bambus[6]uril 12 S14-S15

Dodecakis (1-(2-ethyl- $\beta$-D-glucopyranoside)-1H-1,2,3-triazol-4-yl)methyl) bambus[6]uril 13 S15

Dodecakis (1-(1- $\beta$-D-glucose)-1H-1,2,3-triazol-4-yl)methyl)bambus[6]uril 14 S16 
Tetrabutylammonium-bromide@Dodecakis(1-(1- $\beta-D-g l u c o s e)-1 H-1,2,3-t r i a z o l-4-y l)$

methyl) bambus[6]uril 15

Ethyl 4-azidobutanoate 7a

1- $\beta$-azido-glucose tetraacetate $7 \mathbf{b}$

2-azidoethyl-2,3,4,6-tetra-O-acetyl- $\beta$-D-glucopyranoside 7c

${ }^{1} \mathrm{H}$ NMR and ${ }^{13} \mathrm{C}$ NMR spectra for compounds: $1,2,3,4,5,6,8 \mathrm{a}, 8 \mathrm{~b}, 8 \mathrm{c}, 9 \mathrm{a}, 10 \mathrm{a}, 9 \mathrm{c}, 11 \mathrm{a}$,

11b, 11c, 12, 13, 14, 15

Crystallographic Data Collection and Structure Determinationof $\mathbf{3}$ and $\mathbf{4}$

Figure S1

Determination of $\mathrm{K}_{\mathrm{a}}$ and thermodynamic parameters of complexes by ITC

Figure S2 ITC of I-binding to $\mathbf{B U} \mathbf{1 2}$ in $\mathrm{K}_{2} \mathrm{HPO}_{4}$

Figure S3

ITC of I-binding to BU 13 in $\mathrm{H}_{2} \mathrm{O}$

Figure $\mathrm{S} 4$

ITC of I- binding to BU 13 in $\mathrm{K}_{2} \mathrm{HPO}_{4}$

Figure S5

ITC of $\mathrm{I}^{-}$binding to $\mathrm{BU} \mathbf{1 4}$ in $\mathrm{H}_{2} \mathrm{O}$

S46

Figure S6

ITC of I' binding to $\mathrm{Br} @ B U$ in $15 \mathrm{H}_{2} \mathrm{O}$

547

References

S47

\section{General Methods and equipment}

Commercially available reagents were purchased from Sigma-Aldrich and were used without further purification. All reactions were performed under inert atmosphere using anhydrous solvents which were dried and distilled before being used. For reactions requiring heating or reflux, heat-on blocks were used. Thin-layer chromatograms (TLC) and flash chromatography separations were respectively performed on precoated silica gel 60 F254 plates $(0.25 \mathrm{~mm}$ ) and on Merck Kieselgel 60 (grading 40-63 $\mu \mathrm{m})$. Microwave synthesis were conducted using a CEM Focused Microwave Discover ${ }^{\circledR}$ SP-X System reactor. The reactions were performed for 2-4 h under magnetic stirring in 10 or $35 \mathrm{~mL}$ sealed Discovered SP vessels closed with Activent ${ }^{\circledR}$ caps. MW material was purchased from CEM. The Dynamic Control method was used for all microwave reactions where the temperature and the pressure were set $\left(\mathrm{P}=50 \mathrm{~W}, \mathrm{~T}=80^{\circ} \mathrm{C}\right.$, PowerMax on). Enhanced microwave synthesis (ENS) method using external cooling was used for the synthesis of the Propargylated Bambusurils. ${ }^{1} \mathrm{H}$ NMR spectra $(400 \mathrm{MHz})$ and ${ }^{13} \mathrm{C}$ NMR spectra $(100 \mathrm{MHz})$ were recorded on a Brucker Avance $400 \mathrm{MHz}$ spectrometer. Chemical shifts and coupling constants are reported in parts per million (ppm) and in Hertz $(\mathrm{Hz})$ respectively. HRMS and electrospray mass spectra (ESIMS) were obtained from an LCT Premier XE using electro spray ionization coupled with a time flight analyser (ESI-TOF) and were performed the Institut de Chimie Organique et Analytique ICOA, Université d'Orléans, France. Infrared spectra (IR) were 
recorded on a Perkin Elmer UAR Two Spectrum spectrometer. Electrospray mass spectra were obtained using an ESI-Quadripole autopurify, Waters (pump: 2545, mass: ZQ2000) mass Spectrometer. Optical rotations were measured on a JASCO P-2000 Polarimeter. Melting point were measured on a Büchi Melting point B540.

\section{$\underline{\text { ITC instrument and experiments }}$}

ITC measurements were performed with a VP-ITC microcalorimeter (Microcal, GEHealthcare). Experiments were carried out in water and in solution of $\mathrm{K}_{2} \mathrm{HPO}_{4}(1.5 \mathrm{mM})$ in mili-Q water at $298.15 \pm$ $0.1 \mathrm{~K}$. Anion binding to BU was investigated via a classical isothermal titration experiment $(10 \mu \mathrm{L}$ additions) and a single injection method (SIM). Injection of solution of sodium iodide was added automatically to the BU solution present in the calorimeter cell while stirring at $307 \mathrm{rpm}$. Integrated heat effects were analyzed by non-linear regression using a single-site binding model (Microcal Origin 7). The experimental data fitted to a theorical titration curve giving the association constant $K_{a}$, the enthalpy of binding $\Delta \mathrm{H}^{\circ}$ and the entropy $\Delta \mathrm{S}^{\circ}$. The free energy $\Delta \mathrm{G}^{\circ}$ was calculated from the equation: $\Delta G^{\circ}=\Delta H^{\circ}-T \Delta S^{\circ}$, where $T$ is the absolute temperature. The first smaller addition $(2 \mu \mathrm{L})$, that was used to compensate for diffusion of guest from the injector during equilibration, was discarded prior to data fitting.

\section{Synthetic Procedures and characterization data}<smiles>C#CCN1C(=O)N(CC#C)C2NC(=O)NC21</smiles>

2,4-dipropargylglycoluril $\underline{1}$ A suspension of 4,5 dihydroxyimidazolidin-2-one ${ }^{1}$ ( $\left.6.9 \mathrm{~g}, 59 \mathrm{mmol}, 4 \mathrm{eq}\right)$ and 1,3 dipropargyl urea ${ }^{2}(2 \mathrm{~g}, 14.7 \mathrm{mmol}, 1 \mathrm{eq})$ in a mixture of $\mathrm{HCl} 37 \%(0.24 \mathrm{~mL})$ in $\mathrm{H}_{2} \mathrm{O}(7.5 \mathrm{~mL})$ was stirred at $105{ }^{\circ} \mathrm{C}$ for $24 \mathrm{~h}$. After cooling at room temperature, the precipitate formed was isolated by filtration and washed with water $(10 \mathrm{~mL})$ to give 1 as a brown solid $(2.5 \mathrm{~g}, 78 \%$ yield $)$. M.p $=220^{\circ} \mathrm{C}$; IR $\left(v_{\max } / \mathrm{cm}^{-1}\right): 3399(\mathrm{C} \equiv \mathrm{C}), 3295(\mathrm{NH}), 1714(\mathrm{CO}), 1475\left(\mathrm{CH}_{2}\right), 637(\mathrm{C} \equiv \mathrm{C}) ;{ }^{1} \mathrm{H}$ NMR (400 MHz, DMSO) $\delta=$ $7.58(2 \mathrm{H}, \mathrm{s}, \mathrm{NH}), 5.33(2 \mathrm{H}, \mathrm{s}, \mathrm{CH}), 4.18\left(2 \mathrm{H}, \mathrm{dd}, \mathrm{AB}\right.$ syst, J = 2.3, $\left.17.9 \mathrm{~Hz}, \mathrm{CH}_{2}\right), 3.71(2 \mathrm{H}, \mathrm{dd}, \mathrm{AB}$ syst, J = 2.3, 17.9 Hz, $\left.\mathrm{CH}_{2}\right), 3.26(2 \mathrm{H}, \mathrm{t}, \mathrm{J}=2.3 \mathrm{~Hz}, \mathrm{C} \equiv \mathrm{CH}) ;{ }^{13} \mathrm{C}$ NMR (100 MHz, DMSO) $\delta=161.2(\mathrm{C}=\mathrm{O}), 156.2$ $(\mathrm{C}=\mathrm{O}), 79.4(\mathrm{C} \equiv \mathrm{CH}), 75.1(\mathrm{C} \equiv \mathrm{CH}), 65.6(\mathrm{CH}), 30.9\left(\mathrm{CH}_{2}\right) ; \mathrm{HRMS}$ (ESI-TOF) $\mathrm{m} / z[\mathrm{M}-\mathrm{H}]^{+}$Calcd for $\mathrm{C}_{10} \mathrm{H}_{9} \mathrm{~N}_{4} \mathrm{O}_{2}$ 217.0726; found 217.0727 . 


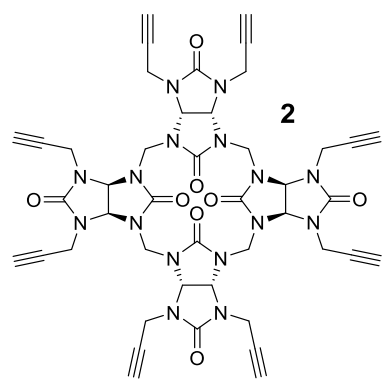

Octapropargylbambus[4] uril 2 A suspension of 2,4-dipropargylglycoluril 3 (100 mg, $0.46 \mathrm{mmol}, 1 \mathrm{eq}$ ), $p$-formaldehyde (14 mg, $0.46 \mathrm{mmol}, 1 \mathrm{eq}$ ) and $p$-toluenesulfonic acid ( $87 \mathrm{mg}, 0.46 \mathrm{mmol}, 1 \mathrm{eq}$ ) in $\mathrm{CHCl}_{3}$ $(4.5 \mathrm{~mL})$ was subjected to microwave irradiations for $3 \mathrm{~h}$ in a $10 \mathrm{ml}$ sealed reactor, under magnetic stirring and continuous cooling with $\mathrm{P}=200 \mathrm{~W}, \mathrm{~T}=75^{\circ} \mathrm{C}$. After concentration under vacuum, the resulting solid was washed with $\mathrm{H}_{2} \mathrm{O}$ and $\mathrm{MeOH}$ to give 2 as a white powder (63.5 mg, $60 \%$ yield). M.p $>300^{\circ} \mathrm{C}$; IR $\left(v_{\max } / \mathrm{cm}^{-1}\right): 3283(\mathrm{C} \equiv \mathrm{C}), 1700(\mathrm{CO}), 1475\left(\mathrm{CH}_{2}\right), 1227(\mathrm{CN}), 650(\mathrm{C} \equiv \mathrm{C}) ;{ }^{1} \mathrm{H}$ NMR $(400 \mathrm{MHz}$, DMSO) $\delta=5.83(8 \mathrm{H}, \mathrm{s}, \mathrm{CH}), 4.97\left(8 \mathrm{H}, \mathrm{s}, \mathrm{CH}_{2}\right.$ bridge $), 4.19\left(8 \mathrm{H}, \mathrm{AB}\right.$ syst, $\left.\mathrm{J}=2.0,18.3 \mathrm{~Hz}, \mathrm{CH}_{2}\right), 4.07(8 \mathrm{H}$, AB syst, $\left.\mathrm{J}=2.0,18.3 \mathrm{~Hz}, \mathrm{CH}_{2}\right), 3.32(8 \mathrm{H}, \mathrm{t}, \mathrm{J}=2.0 \mathrm{~Hz}, \mathrm{C} \equiv \mathrm{CH}) ;{ }^{13} \mathrm{C}$ NMR (100 MHz, Acetone) $\delta=160.6$ $(\mathrm{C}=\mathrm{O}), 157.2(\mathrm{C}=\mathrm{O}), 79.9(\mathrm{C} \equiv \mathrm{CH}), 74.4(\mathrm{C} \equiv \mathrm{CH}), 72.1(\mathrm{CH}), 52.5\left(\mathrm{CH}_{2}\right.$ bridge), $33.2\left(\mathrm{CH}_{2}\right) ; \mathrm{HRMS}$ (ESI-TOF) $\mathrm{m} / z[\mathrm{M}+\mathrm{H}]^{+}$Calcd for $\mathrm{C}_{44} \mathrm{H}_{41} \mathrm{~N}_{16} \mathrm{O}_{8}$ 921.3293, Found 921.3295 .

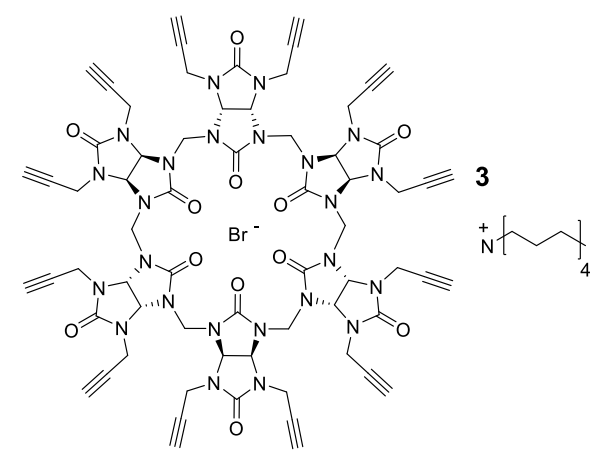

Tetrabutylammonium bromide @Dodecapropargylbambus[6]uril $\underline{\mathbf{3}}$ A suspension of 2,4dipropargylglycoluril 1 (220 mg, $1.0 \mathrm{mmol}, 1 \mathrm{eq}), p$-formaldehyde (45.5 mg, $1.52 \mathrm{mmol}, 1.5 \mathrm{eq}), p$ toluenesulfonic acid ( $96.1 \mathrm{mg}, 0.51 \mathrm{mmol}, 0.5 \mathrm{eq}$ ) and $\operatorname{TBABr}\left(52.4 \mathrm{mg}, 0.16 \mathrm{mmol}, 0.16 \mathrm{eq}\right.$ ) in $\mathrm{CHCl}_{3}$ $(10 \mathrm{~mL})$ was subjected to microwave irradiations for $2 \mathrm{~h}$ in a $35 \mathrm{~mL}$ sealed reactor under magnetic stirring and continously cooling with $\mathrm{P}=200 \mathrm{~W}, \mathrm{~T}=75^{\circ} \mathrm{C}$. After concentration, the resulting crude was purified by silicagel column chromatography $\left(\mathrm{CH}_{2} \mathrm{Cl}_{2} /\right.$ Acetone $\left.(7 / 3)\right)$ to give $\mathbf{3}$ as a white solid (128.7

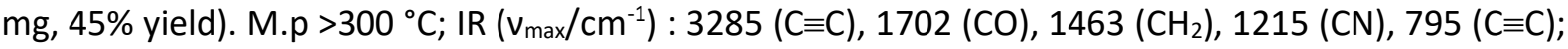
${ }^{1} \mathrm{H}$ NMR (400 MHz, Acetone) $\delta=5.82(12 \mathrm{H}, \mathrm{s}, \mathrm{CH}), 5.26\left(12 \mathrm{H}, \mathrm{s}, \mathrm{CH}_{2}\right.$ bridge), $4.51(12 \mathrm{H}, \mathrm{AB}$ syst, J = 2.1, $\left.18.0 \mathrm{~Hz}, \mathrm{CH}_{2}\right), 4.44\left(12 \mathrm{H}, \mathrm{AB}\right.$ syst, J = 2.1, $\left.18.0 \mathrm{~Hz}, \mathrm{CH}_{2}\right), 3.46\left(8 \mathrm{H}, \mathrm{m}, \mathrm{N}-\mathrm{CH}_{2}-\mathrm{CH}_{2}-\mathrm{CH}_{2}-\mathrm{CH}_{3}\right), 2.51(12 \mathrm{H}, \mathrm{t}$, $\mathrm{J}=2.6 \mathrm{~Hz}, \mathrm{C} \equiv \mathrm{CH}), 1.84\left(8 \mathrm{H}, \mathrm{m}, \mathrm{N}-\mathrm{CH}_{2}-\mathrm{CH}_{2}-\mathrm{CH}_{2}-\mathrm{CH}_{3}\right), 1.44\left(8 \mathrm{H}, \mathrm{q}, \mathrm{J}=7.4 \mathrm{~Hz}, \mathrm{~N}-\mathrm{CH}_{2}-\mathrm{CH}_{2}-\mathrm{CH}_{2}-\mathrm{CH}_{3}\right), 0.98$ $\left(12 \mathrm{H}, \mathrm{t}, \mathrm{J}=7.4 \mathrm{~Hz}, \mathrm{~N}-\mathrm{CH}_{2}-\mathrm{CH}_{2}-\mathrm{CH}_{2}-\mathrm{CH}_{3}\right) ;{ }^{13} \mathrm{C} \mathrm{NMR}(100 \mathrm{MHz}$, Acetone) $\delta=159.7$ (C=0), 159.1 (C=0), 81.4 $(\mathrm{C} \equiv \mathrm{CH}), 72.9(\mathrm{C} \equiv \mathrm{CH}), 68.0(\mathrm{CH}), 55.0\left(\mathrm{~N}-\mathrm{CH}_{2}-\mathrm{CH}_{2}-\mathrm{CH}_{2}-\mathrm{CH}_{3}\right), 48.5\left(\mathrm{CH}_{2}\right.$ bridge), $34.4\left(\mathrm{CH}_{2}\right), 24.5\left(\mathrm{~N}-\mathrm{CH}_{2}-\right.$ $\left.\mathrm{CH}_{2}-\mathrm{CH}_{2}-\mathrm{CH}_{3}\right), 20.4\left(\mathrm{~N}-\mathrm{CH}_{2}-\mathrm{CH}_{2}-\mathrm{CH}_{2}-\mathrm{CH}_{3}\right), 13.9\left(\mathrm{~N}-\mathrm{CH}_{2}-\mathrm{CH}_{2}-\mathrm{CH}_{2}-\mathrm{CH}_{3}\right) ; \mathrm{HRMS}$ (ESI-TOF) m/z [M -TBA + H] ${ }^{+}$ Calcd for $\mathrm{C}_{66} \mathrm{H}_{61} \mathrm{BrN}_{24} \mathrm{O}_{12}$ 1461.4157; found 1461.4155. Crystallographic Information File (CIF) of 3 is saved in text format at the end of the Supporting Information File (S43); The crystal structure has been deposited at the Cambridge Crystallographic Data Centre and allocated the deposition number CCDC 1874615. 


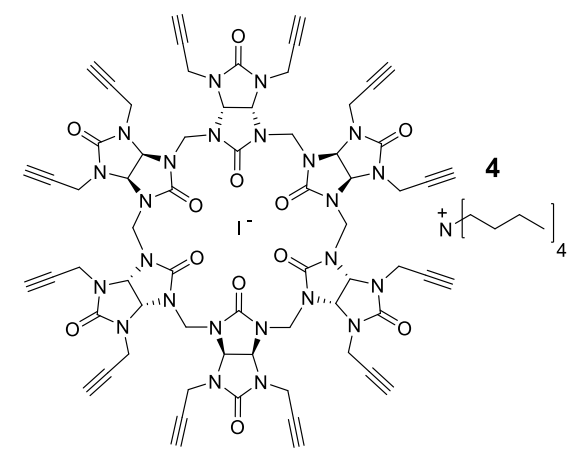

Tetrabutylammonium iodide @Dodecapropargylbambus[6]uril 4 A suspension of 2,4dipropargylglycoluril 1 (100 mg, $0.46 \mathrm{mmol}, 1 \mathrm{eq}), p$-formaldehyde $(20.7 \mathrm{mg}, 0.69 \mathrm{mmol}, 1.5 \mathrm{eq}), p$ toluenesulfonic acid ( $43.7 \mathrm{mg}, 0.23 \mathrm{mmol}, 0.5 \mathrm{eq}$ ) and TBAI ( $27 \mathrm{mg}, 0.074 \mathrm{mmol}, 0.16 \mathrm{eq}$ ) in $\mathrm{CHCl}_{3}(4.5$ $\mathrm{mL}$ ) was subjected to microwave radiations for $2 \mathrm{~h}$ in a $10 \mathrm{ml}$ sealed reactor, under magnetic stirring and continuous stirring with $\mathrm{P}=200 \mathrm{~W}, \mathrm{~T}=75^{\circ} \mathrm{C}$. After concentration, the resulting crude was purified by silicagel column chromatography $\left(\mathrm{CH}_{2} \mathrm{Cl}_{2} /\right.$ Acetone (7/3)) to give 4 as a white solid $(75.1 \mathrm{mg}, 56 \%$ yield). M.p >300 ${ }^{\circ} \mathrm{C}$; IR $\left(\mathrm{V}_{\max } / \mathrm{cm}^{-1}\right): 3285(\mathrm{C} \equiv \mathrm{C}), 1698(\mathrm{CO}), 1462\left(\mathrm{CH}_{2}\right), 1214(\mathrm{CN}), 794(\mathrm{C} \equiv \mathrm{C}) ;{ }^{1} \mathrm{H}$ NMR (400 MHz, Acetone) $\delta=5.93(12 \mathrm{H}, \mathrm{s}, \mathrm{CH}), 5.25\left(12 \mathrm{H}, \mathrm{s}, \mathrm{CH}_{2}\right.$ bridge), $4.51(12 \mathrm{H}, \mathrm{AB}$ syst, $\mathrm{J}=2.2,18.0 \mathrm{~Hz}$, $\left.\mathrm{CH}_{2}\right), 4.46\left(12 \mathrm{H}, \mathrm{AB}\right.$ syst, $\left.\mathrm{J}=2.2,18.0 \mathrm{~Hz}, \mathrm{CH}_{2}\right), 3.46\left(8 \mathrm{H}, \mathrm{t}, \mathrm{J}=8.6 \mathrm{~Hz}, \mathrm{~N}-\mathrm{CH}_{2}-\mathrm{CH}_{2}-\mathrm{CH}_{2}-\mathrm{CH}_{3}\right), 2.52(12 \mathrm{H}, \mathrm{t}$, $\mathrm{J}=2.2 \mathrm{~Hz}, \mathrm{C} \equiv \mathrm{CH}), 1.83\left(8 \mathrm{H}, \mathrm{m}, \mathrm{N}-\mathrm{CH}_{2}-\mathrm{CH}_{2}-\mathrm{CH}_{2}-\mathrm{CH}_{3}\right), 1.44\left(8 \mathrm{H}, \mathrm{q}, \mathrm{J}=7.4 \mathrm{~Hz}, \mathrm{~N}-\mathrm{CH}_{2}-\mathrm{CH}_{2}-\mathrm{CH}_{2}-\mathrm{CH}_{3}\right), 0.98$ $\left(12 \mathrm{H}, \mathrm{t}, \mathrm{J}=7.4 \mathrm{~Hz}, \mathrm{~N}-\mathrm{CH}_{2}-\mathrm{CH}_{2}-\mathrm{CH}_{2}-\mathrm{CH}_{3}\right) ;{ }^{13} \mathrm{C} \mathrm{NMR}(100 \mathrm{MHz}$, Acetone) $\delta=159.9$ (C=O), 159.4 (C=O), 81.5 $(\mathrm{C} \equiv \mathrm{CH}), 72.9(\mathrm{C} \equiv \mathrm{CH}), 68.7(\mathrm{CH}), 59.5\left(\mathrm{~N}-\mathrm{CH}_{2}-\mathrm{CH}_{2}-\mathrm{CH}_{2}-\mathrm{CH}_{3}\right), 48.5\left(\mathrm{CH}_{2}\right.$ bridge $), 34.7\left(\mathrm{CH}_{2}\right), 24.5\left(\mathrm{~N}-\mathrm{CH}_{2}-\right.$ $\left.\mathrm{CH}_{2}-\mathrm{CH}_{2}-\mathrm{CH}_{3}\right), 20.5\left(\mathrm{~N}-\mathrm{CH}_{2}-\mathrm{CH}_{2}-\mathrm{CH}_{2}-\mathrm{CH}_{3}\right), 13.9\left(\mathrm{~N}^{-} \mathrm{CH}_{2}-\mathrm{CH}_{2}-\mathrm{CH}_{2}-\mathrm{CH}_{3}\right) ; \mathrm{HRMS}$ (ESI-TOF) $\mathrm{m} / z$ [M -TBA+H] ${ }^{+}$ Calcd for $\mathrm{C}_{66} \mathrm{H}_{61} \mathrm{~N}_{24} \mathrm{O}_{12} \mathrm{I}$ 1509.4018; found 1509.4012. Crystallographic Information File (CIF) of 3 is saved in text format at the end of the Supporting Information File (S43); The crystal structure has been deposited at the Cambridge Crystallographic Data Centre and allocated the deposition number CCDC 1874616;

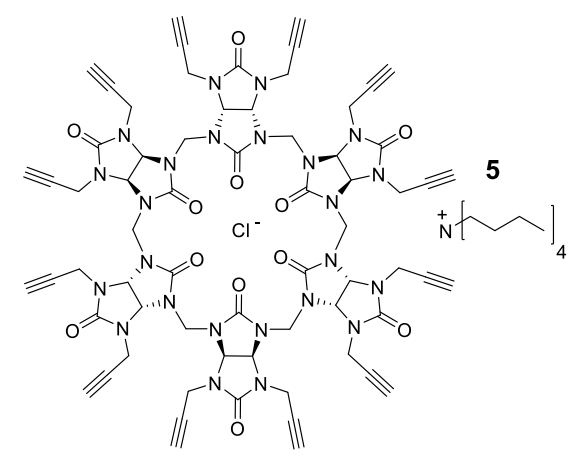

Tetrabutylammonium chloride @dodecapropargylbambus[6]uril $\underline{\mathbf{5}}$ A suspension of 2,4dipropargylglycoluril 1 (100.0 mg, $0.46 \mathrm{mmol}, 1 \mathrm{eq}), p$-formaldehyde $(20.7 \mathrm{mg}, 0.69 \mathrm{mmol}, 1.5 \mathrm{eq}), p$ toluenesulfonic acid $(43.7 \mathrm{mg}, 0.23 \mathrm{mmol}, 0.5 \mathrm{eq})$ and TBACl $(20.5 \mathrm{mg}, 74.00 \mu \mathrm{mol}, 0.16 \mathrm{eq})$ in $\mathrm{CHCl}_{3}$ $(4.5 \mathrm{~mL})$ was subjected to microwave radiations for $2 \mathrm{~h}$ in a $10 \mathrm{ml}$ sealed reactor, under magnetic stirring and continuous stirring with $P=200 \mathrm{~W}, \mathrm{~T}=75^{\circ} \mathrm{C}$. After concentration, the resulting crude was purified by silicagel column chromatography $\left(\mathrm{CH}_{2} \mathrm{Cl}_{2} /\right.$ Acetone $\left.(7 / 3)\right)$ to give 5 as a white solid $(24.1 \mathrm{mg}$, 19 \%). M.p > $300{ }^{\circ} \mathrm{C} ; \mathrm{IR}\left(\mathrm{v}_{\max } / \mathrm{cm}^{-1}\right): 3285(\mathrm{C} \equiv \mathrm{C}), 1699(\mathrm{CO}), 1464\left(\mathrm{CH}_{2}\right), 1215(\mathrm{CN}), 795(\mathrm{C} \equiv \mathrm{C}) .{ }^{1} \mathrm{H}$ NMR (400 MHz, Acetone): $\delta(p p m)=5.76(12 \mathrm{H}, \mathrm{s}, \mathrm{CH}), 5.28\left(12 \mathrm{H}, \mathrm{s}, \mathrm{CH}_{2}\right.$ bridge), $4.53(12 \mathrm{H}, \mathrm{AB}$ syst, $J=2.3$, $\left.18.0 \mathrm{~Hz}, \mathrm{CH}_{2}-\mathrm{C} \equiv \mathrm{CH}\right), 4.44\left(12 \mathrm{H}, \mathrm{AB}\right.$ syst, $\left.J=2.3,18.0 \mathrm{~Hz}, \mathrm{CH}_{2^{-}} \mathrm{C} \equiv \mathrm{CH}\right), 3.46\left(8 \mathrm{H}, \mathrm{t}, J=8.6 \mathrm{~Hz}, \mathrm{~N}-\mathrm{CH}_{2^{-}} \mathrm{CH}_{2^{-}}\right.$ 
$\left.\mathrm{CH}_{2}-\mathrm{CH}_{3}\right), 2.50(12 \mathrm{H}, \mathrm{t}, \mathrm{J}=2.2 \mathrm{~Hz}, \mathrm{C} \equiv \mathrm{CH}), 1.83\left(8 \mathrm{H}, \mathrm{m}, \mathrm{N}-\mathrm{CH}_{2}-\mathrm{CH}_{2}-\mathrm{CH}_{2}-\mathrm{CH}_{3}\right), 1.44\left(8 \mathrm{H}, \mathrm{q}, \mathrm{J}=7.4, \mathrm{~N}-\mathrm{CH}_{2}-\right.$ $\left.\mathrm{CH}_{2}-\mathrm{CH}_{2}-\mathrm{CH}_{3}\right), 0.98\left(12 \mathrm{H}, \mathrm{t}, J=7.4 \mathrm{~Hz}, \mathrm{~N}-\mathrm{CH}_{2}-\mathrm{CH}_{2}-\mathrm{CH}_{2}-\mathrm{CH}_{3}\right) .{ }^{13} \mathrm{C} \mathrm{NMR}(100 \mathrm{MHz}$, Acetone): $\delta(p p m)=$ $159.6(\mathrm{CO}), 158.8(\mathrm{CO}), 81.3(\mathrm{C} \equiv \mathrm{CH}), 72.9(\mathrm{C} \equiv \mathrm{CH}), 67.7(\mathrm{CH}), 59.4\left(\mathrm{~N}-\mathrm{CH}_{2}-\mathrm{CH}_{2}-\mathrm{CH}_{2}-\mathrm{CH}_{3}\right), 48.6\left(\mathrm{CH}_{2}\right.$ bridge), $34.2\left(\mathrm{CH}_{2}\right), 24.4\left(\mathrm{~N}-\mathrm{CH}_{2}-\mathrm{CH}_{2}-\mathrm{CH}_{2}-\mathrm{CH}_{3}\right), 20.4\left(\mathrm{~N}-\mathrm{CH}_{2}-\mathrm{CH}_{2}-\mathrm{CH}_{2}-\mathrm{CH}_{3}\right), 13.8\left(\mathrm{~N}^{-} \mathrm{CH}_{2}-\mathrm{CH}_{2}-\mathrm{CH}_{2}-\mathrm{CH}_{3}\right)$. HRMS (ESI-TOF) $m / z$ [M -TBA + Cl-H] ${ }^{+}$Calcd for $\mathrm{C}_{66} \mathrm{H}_{61} \mathrm{ClN}_{24} \mathrm{O}_{12}$ 1381.4895; found 1381.4905.

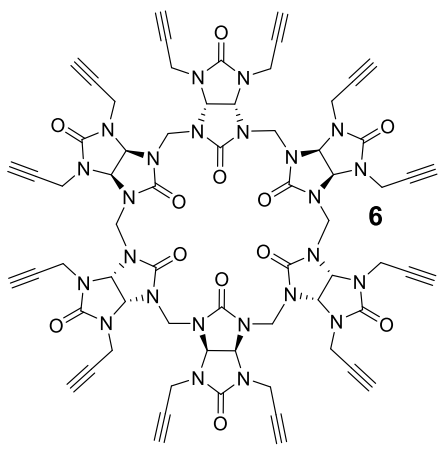

Dodecapropargylbambus[6]uril $\underline{\mathbf{6}}$ To a solution of tetrabutylammonium iodide @Dodecapropargylbambus[6]uril $\underline{4}$ ( $50 \mathrm{mg}, 28.6 \mu \mathrm{mol}, 1 \mathrm{eq})$ in $\mathrm{MeOH}(2 \mathrm{~mL})$ was added $\mathrm{AgSbF}_{6}(16.7$ $\mathrm{mg}, 57.2 \mu \mathrm{mol}, 2 \mathrm{eq})$ to generate the formation of a precipitate. The resulting mixture was centrifugated at $6500 \mathrm{rpm}$ for $5 \mathrm{~min}$. The solvent was discarded and the resulting precipitate was washed three times with $\mathrm{NH}_{4} \mathrm{OH}(2 \mathrm{~mL})$ to yield anion-free dodecapropargylbambus[6] uril 6 as a white solid (35.4 mg, 90\% yield). M.p> 300 $\mathrm{C}$; IR $\left(\mathrm{v}_{\max } / \mathrm{cm}^{-1}\right): 3285(\mathrm{C} \equiv \mathrm{C}), 1702(\mathrm{CO}), 1467\left(\mathrm{CH}_{2}\right), 1216(\mathrm{CN})$, $655(\mathrm{C} \equiv \mathrm{C}) ;{ }^{1} \mathrm{H}$ NMR $(400 \mathrm{MHz}, \mathrm{DMSO}): \delta=5.54(12 \mathrm{H}, \mathrm{s}, \mathrm{CH}), 5.16\left(12 \mathrm{H}, \mathrm{s}, \mathrm{CH}_{2}\right.$ bridge), 4.36-4.23 (24H, $A B$ syst, $\left.\mathrm{J}=2.0,18.0 \mathrm{~Hz}, \mathrm{CH}_{2}-\right), 4.44\left(12 \mathrm{H}, \mathrm{AB}\right.$ syst, $\left.\mathrm{J}=2.2,18.0 \mathrm{~Hz}, \mathrm{CH}_{2}\right), 2.98(12 \mathrm{H}, \mathrm{s}, \mathrm{C} \equiv \mathrm{CH}) ;{ }^{13} \mathrm{C} N M R$ (100 MHz, Acetone) $\delta=159.9(\mathrm{C}=0), 159.0(\mathrm{C}=\mathrm{O}), 81.3(\mathrm{C} \equiv \mathrm{CH}), 72.9(\mathrm{C} \equiv \mathrm{CH}), 67.9(\mathrm{CH}), 48.5\left(\mathrm{CH}_{2}\right.$ bridge), $34.3\left(\mathrm{CH}_{2}\right)$; HRMS (ESI-TOF) $\mathrm{m} / 2[\mathrm{M}+\mathrm{H}]^{+}$Calcd for $\mathrm{C}_{66} \mathrm{H}_{61} \mathrm{~N}_{24} \mathrm{O}_{12}$ 1381.4895; found 1381.4889.

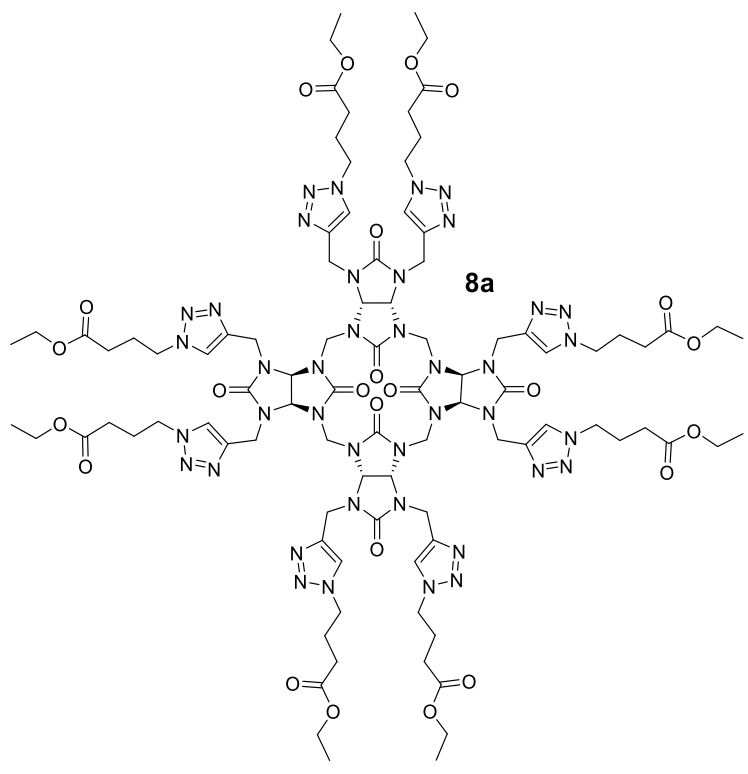

Octakis (1-((4-ethoxy-4-oxobutyl)-1H-1,2,3-triazol-4-yl)methyl) bambus[4]uril 8a To a solution of octapropargylbambus[4]uril $2(24.6 \mathrm{mg}, 26.7 \mu \mathrm{mol}, 1 \mathrm{eq})$ and ethyl 4-azidobutanoate $7 \mathrm{a}^{3}$ (33.5 mg, 
$0.21 \mathrm{mmol}, 8 \mathrm{eq})$ in DMSO $(0.5 \mathrm{~mL})$ was added a solution of sodium ascorbate $(5.3 \mathrm{mg}, 26.7 \mu \mathrm{mol}, 1$ eq) and $\mathrm{CuSO}_{4} .5 \mathrm{H}_{2} \mathrm{O}(2.6 \mathrm{mg}, 11 \mu \mathrm{mol}, 0.4 \mathrm{eq})$ in $\mathrm{H}_{2} \mathrm{O} / t-\mathrm{BuOH}(2.5 / 3 \mathrm{~mL})$. The reaction was heated at $40{ }^{\circ} \mathrm{C}$ for $24 \mathrm{~h}$. The reaction mixture was diluted with $\mathrm{CH}_{2} \mathrm{Cl}_{2}(3 \mathrm{~mL})$ and followed by addition of an EDTA solution (0.1 M in water, $1 \mathrm{~mL}$ ). The organic layer was separated, washed with $\mathrm{H}_{2} \mathrm{O}$, dried over $\mathrm{Na}_{2} \mathrm{SO}_{4}$ and concentrated under vacuum. The crude residue was purified by silicagel column chromatography $\left(\mathrm{CH}_{2} \mathrm{Cl}_{2} / \mathrm{MeOH}(9 / 1)\right)$, to afford $8 \mathrm{a}\left(29.8 \mathrm{mg}, 51 \%\right.$ yield). IR $\left(\mathrm{v}_{\max } / \mathrm{cm}^{-1}\right): 1722$ (CO ester), 1700 (CO), $1476\left(\mathrm{CH}_{2}\right), 1216(\mathrm{CN}) ;{ }^{1} \mathrm{H}$ NMR $\left(400 \mathrm{MHz}, \mathrm{CDCl}_{3}\right) \delta=7.60(8 \mathrm{H}, \mathrm{s}, \mathrm{N}-\mathrm{CH}=\mathrm{C}-\mathrm{N}), 5.74(8 \mathrm{H}, \mathrm{s}, \mathrm{CH}), 4.64-4.57$ $\left(24 \mathrm{H}, \mathrm{m}, \mathrm{CH}_{2}\right.$ bridge, $\left.\mathrm{CH}_{2}\right), 4.43\left(16 \mathrm{H}, \mathrm{dd}, \mathrm{J}=6.6,11.2 \mathrm{~Hz}, \mathrm{~N}-\mathrm{CH}_{2}-\left(\mathrm{CH}_{2}\right)_{2}-\mathrm{C}=\mathrm{O}\right), 4.10(16 \mathrm{H}, \mathrm{q}, \mathrm{J}=7.1 \mathrm{~Hz}$, $\left.\mathrm{O}-\mathrm{CH}_{2}-\mathrm{CH}_{3}\right), 2.34\left(16 \mathrm{H}, \mathrm{t}, \mathrm{J}=8 \mathrm{~Hz}, \mathrm{~N}-\left(\mathrm{CH}_{2}\right)_{2}-\mathrm{CH}_{2}-\mathrm{C}=\mathrm{O}\right), 2.18\left(16 \mathrm{H}, \mathrm{m}, \mathrm{N}-\mathrm{CH}_{2}-\mathrm{CH}_{2}-\mathrm{CH}_{2}-\mathrm{C}=\mathrm{O}\right), 1.24(24 \mathrm{H}, \mathrm{t}$, $\left.\mathrm{J}=7.1 \mathrm{~Hz}, \mathrm{O}-\mathrm{CH}_{2}-\mathrm{CH}_{3}\right) ;{ }^{13} \mathrm{C} \mathrm{NMR}\left(100 \mathrm{MHz}, \mathrm{CDCl}_{3}\right) \delta=172.5$ (C=O ester), 159.7 (C=O), 158.3 (C=O), 143.9 $(\mathrm{N}-\mathrm{CH}=\mathrm{C}-\mathrm{N}), 123.0(\mathrm{~N}-\mathrm{CH}=\mathrm{C}-\mathrm{N}), 71.4(\mathrm{CH}), 60.8\left(\mathrm{O}-\mathrm{CH}_{2}-\mathrm{CH}_{3}\right), 50.7\left(\mathrm{CH}_{2}\right.$ bridge $), 49.5\left(\mathrm{~N}^{-} \mathrm{CH}_{2}-\left(\mathrm{CH}_{2}\right)_{2}-\mathrm{C}=\mathrm{O}\right)$, $38.8\left(\mathrm{CH}_{2}\right), 30.9\left(\mathrm{~N}-\left(\mathrm{CH}_{2}\right)_{2}-\mathrm{CH}_{2}-\mathrm{C}=\mathrm{O}\right), 25.5\left(\mathrm{~N}-\mathrm{CH}_{2}-\mathrm{CH}_{2}-\mathrm{CH}_{2}-\mathrm{C}=\mathrm{O}\right), 14.3\left(\mathrm{O}-\mathrm{CH}_{2}-\mathrm{CH}_{3}\right) ; \mathrm{HRMS}$ (ESI-TOF) $\mathrm{m} / \mathrm{z}$ $[\mathrm{M}+2 \mathrm{H}]^{2+}$ Calcd for $\mathrm{C}_{92} \mathrm{H}_{130} \mathrm{~N}_{40} \mathrm{O}_{24}$ 1089.5085; found 1089.5095 .

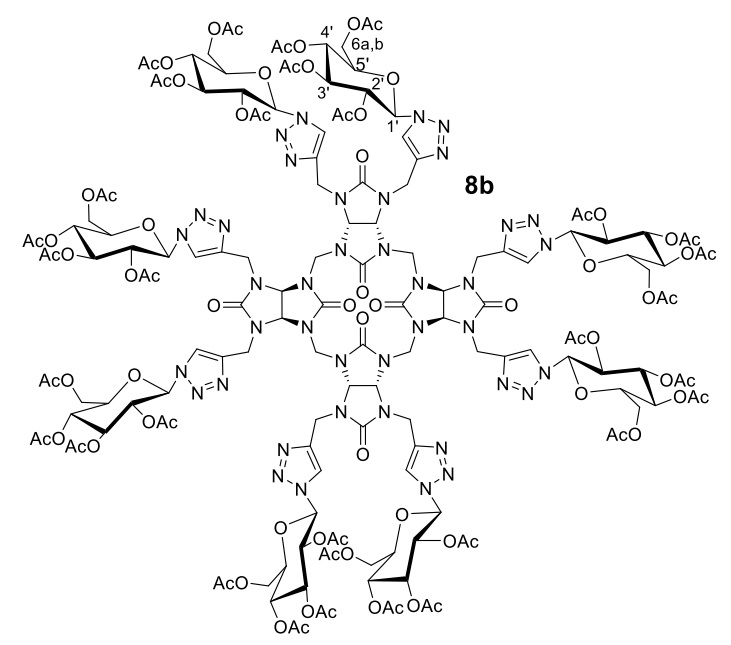

Octakis (1-(1- $\beta$-tetraacetylated glucose)-1H-1,2,3-triazol-4-yl)methyl) bambus[4]uril $\underline{8 b}$ To a solution of octapropargylbambus[4]uril 2 (32.1 mg, $35.0 \mu \mathrm{mol}, 1 \mathrm{eq}$ ) and 1- $\beta$-azido-2,3,4,6 -tetra-O-acetyl-Dglucopyranoside $7 \mathrm{~b}^{4}(118.0 \mathrm{mg}, 0.32 \mathrm{mmol}, 9 \mathrm{eq})$ in DMSO $(0.5 \mathrm{~mL})$, was added a solution of sodium ascorbate $1 \mathrm{M}$ in water $(70 \mu \mathrm{L}, 70.0 \mu \mathrm{mol}, 2 \mathrm{eq}$ ) and copper sulfate $1 \mathrm{M}$ in water ( $35 \mu \mathrm{L}, 35.0 \mu \mathrm{mol}, 1$ eq) in $\mathrm{H}_{2} \mathrm{O} / \mathrm{tBuOH}(1 / 1.2 \mathrm{~mL})$. The reaction was subjected to microwave radiations for $2 \mathrm{~h}$ in a pressurized reactor, under magnetic stirring with $\mathrm{P}=50 \mathrm{~W}, \mathrm{~T}=80^{\circ} \mathrm{C}$. The mixture was concentrated under reduced pressure, diluted in a mixture of $\mathrm{MeCN} / \mathrm{H}_{2} \mathrm{O} / \mathrm{NH}_{4} \mathrm{OH}(9: 1: 1)$ and filtered with the same eluent $(25 \mathrm{~mL})$ on a small pad of $\mathrm{SiO}_{2}$. The filtrate was evaporated under reduced pressure and then purified by silicagel column chromatography $\left(\mathrm{CH}_{2} \mathrm{Cl}_{2} / \mathrm{MeOH}(9 / 1)\right)$ to afford $\mathbf{8 b}(73.8 \mathrm{mg}, 54 \%$ yield). $[\alpha]_{\mathrm{D}}-13.7$ (c 0.25 in $\left.\mathrm{CHCl}_{3}\right)$; IR $\left(\mathrm{v}_{\max } / \mathrm{cm}^{-1}\right): 1749(\mathrm{CO}), 1480\left(\mathrm{CH}_{2}\right), 1217(\mathrm{CN}), 1038(\mathrm{CO}) ;{ }^{1} \mathrm{H}$ NMR (400 $\left.\mathrm{MHz}, \mathrm{CDCl}_{3}\right) \delta=8.21-8.07(8 \mathrm{H}, \mathrm{br} \mathrm{s}, \mathrm{N}-\mathrm{CH}=\mathrm{C}-\mathrm{N}), 6.07-5.86\left(8 \mathrm{H}, \mathrm{d}, \mathrm{J}=8.6 \mathrm{~Hz}, \mathrm{H} 1^{\prime}\right), 5.47-5.27(32 \mathrm{H}, \mathrm{m}$, $\mathrm{CH}, \mathrm{H}^{\prime}, \mathrm{H3}^{\prime}, \mathrm{H} 4^{\prime}$ ), 4.69-4.56 (24H, m, CH $2, \mathrm{CH}_{2}$ bridge), 4.29-4.03 (24H, m, H5', H6a, b), 2.05-2.02-1.96$1.75\left(96 \mathrm{H}, \mathrm{s}, \mathrm{CH}_{3}\right) ;{ }^{13} \mathrm{C} \mathrm{NMR}\left(100 \mathrm{MHz}, \mathrm{CDCl}_{3}\right) \delta=170.6-170.0-169.6-168.9$ (C=O OAC), 159.6 (C=O), $159.0(\mathrm{C}=\mathrm{O}), 145.0(\mathrm{~N}-\mathrm{CH}=\mathrm{C}-\mathrm{N}), 122.0(\mathrm{~N}-\mathrm{CH}=\mathrm{C}-\mathrm{N}), 85.7\left(\mathrm{C1}^{\prime}\right), 75.0\left(\mathrm{C5}^{\prime}\right), 72.6(\mathrm{CH}), 70.7\left(\mathrm{C3}^{\prime}\right), 67.8$ (C2'), $61.8\left(\mathrm{C}^{\prime}\right), 52.9\left(\mathrm{C}_{\mathrm{a}, \mathrm{b}}\right), 49.9\left(\mathrm{CH}_{2}\right.$ bridge), $38.2\left(\mathrm{CH}_{2}\right), 20.7-20.6-20.1\left(\mathrm{CH}_{3}\right) ; \mathrm{HRMS}$ (ESI-TOF) $\mathrm{m} / \mathrm{z}$ $[\mathrm{M}+2 \mathrm{H}]^{2+}$ Calcd for $\mathrm{C}_{156} \mathrm{H}_{194} \mathrm{~N}_{40} \mathrm{O}_{80}$ 1953.6165; found 1953.6170 . 


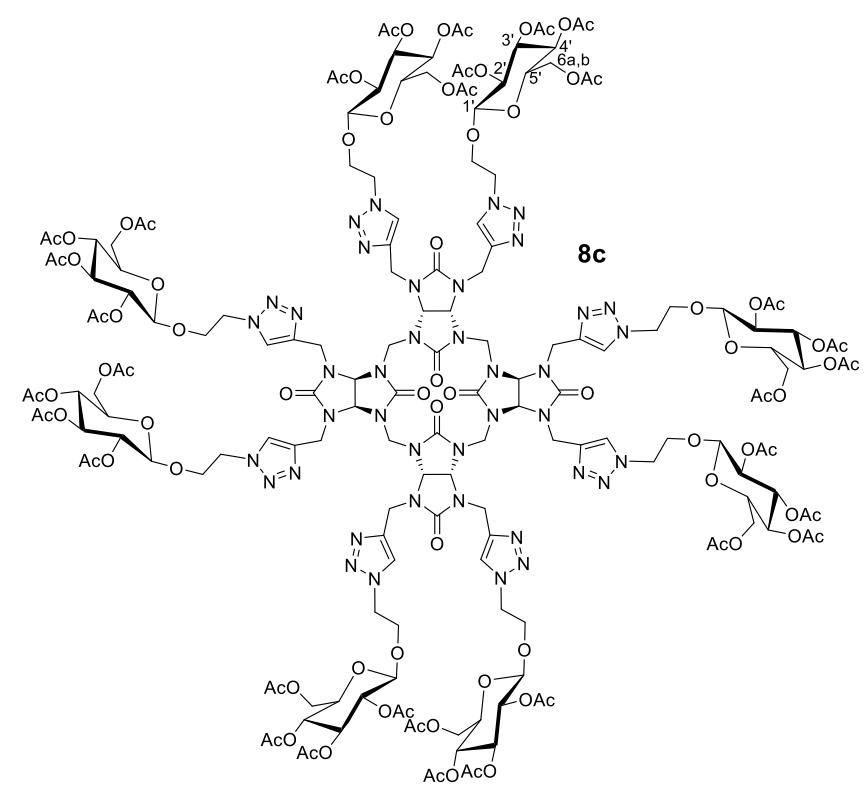

Octakis(1-(2-ethyl-2,3,4,6-tetra-O-acetyl- $\beta$-D-glucopyranoside)-1H-1,2,3-triazol-4-yl)methyl)

bambus[4]uril $\underline{8 c}$ To a solution of octapropargylbambus[4]uril $4(24.0 \mathrm{mg}, 26.0 \mu \mathrm{mol}, 1 \mathrm{eq})$ and 2-azidoethyl-2,3,4,6-tetra-O-acetyl- $\beta$-D-glucopyranoside $7 \mathrm{c}^{5}(97.6 \mathrm{mg}, 0.23 \mathrm{mmol}, 9 \mathrm{eq})$ in DMSO (0.5 $\mathrm{mL})$, was added a solution of sodium ascorbate $1 \mathrm{M}$ in water $(52 \mu \mathrm{L}, 52.0 \mu \mathrm{mol}, 2 \mathrm{eq})$ and copper sulfate $1 \mathrm{M}$ in water ( $26 \mu \mathrm{L}, 26.0 \mu \mathrm{mol}, 1 \mathrm{eq})$ in $\mathrm{H}_{2} \mathrm{O} / \mathrm{tBuOH}(1 / 1.2 \mathrm{~mL})$. The reaction was subjected to microwave radiations for $2 \mathrm{~h}$ in a pressurized reactor, under magnetic stirring with $\mathrm{P}=50 \mathrm{~W}, \mathrm{~T}=80^{\circ} \mathrm{C}$. The mixture was concentrated under reduced pressure, diluted in a mixture of $\mathrm{MeCN} / \mathrm{H}_{2} \mathrm{O} / \mathrm{NH}_{4} \mathrm{OH}$ (9:1:1) and filtered with the same eluent $(25 \mathrm{~mL})$ on a small pad of $\mathrm{SiO}_{2}$. The filtrate was evaporated under reduced pressure and then purified by silicagel column chromatography $\left(\mathrm{CH}_{2} \mathrm{Cl}_{2} / \mathrm{MeOH}(9 / 1)\right)$ to afford 8c $(69.8 \mathrm{mg}, 63 \% \text { yield). [ } \alpha]_{\mathrm{D}}=-12.5$ (c $\left.0.2545 \mathrm{in} \mathrm{CHCl}_{3}\right)$; IR $\left(\mathrm{v}_{\max } / \mathrm{cm}^{-1}\right): 1746(\mathrm{CO}), 1474\left(\mathrm{CH}_{2}\right)$, 1215 (CN), 1036 (CO); ${ }^{1} \mathrm{H} \mathrm{NMR}\left(400 \mathrm{MHz}, \mathrm{CDCl}_{3}\right) \delta=7.57$ (4H, s, N-CH=C-N), $7.6(4 \mathrm{H}, \mathrm{s}, \mathrm{N}-\mathrm{CH}=\mathrm{C}-\mathrm{N}$ ), 5.68 $(8 \mathrm{H}, \mathrm{s}, \mathrm{CH}), 5.28-5.16\left(8 \mathrm{H}, \mathrm{m}, \mathrm{H3} 3^{\prime}\right), 5.08-5.05\left(8 \mathrm{H}, \mathrm{m}, \mathrm{H} 4^{\prime}\right), 4.98-4.92\left(8 \mathrm{H}, \mathrm{m}, \mathrm{H} 2^{\prime}\right), 4.58-4.47(48 \mathrm{H}, \mathrm{m}$, $\mathrm{CH}_{2}$ bridge, $\left.\mathrm{N}-\mathrm{CH}_{2}-\mathrm{CH}_{2}-\mathrm{O}, \mathrm{CH}_{2}, \mathrm{H} 1^{\prime}\right), 4.27-4.09\left(24 \mathrm{H}, \mathrm{m}, \mathrm{H} 6 \mathrm{a}, \mathrm{N}-\mathrm{CH}_{2}-\mathrm{CH}_{2}-\mathrm{O}\right), 3.95(8 \mathrm{H}, \mathrm{m}, \mathrm{H} 6 \mathrm{~b}), 3.72(8 \mathrm{H}$, $\left.\mathrm{m}, \mathrm{H} 5^{\prime}\right), 2.07-1.93\left(96 \mathrm{H}, \mathrm{s}, \mathrm{CH}_{3}\right) ;{ }^{13} \mathrm{C}$ NMR $\left(100 \mathrm{MHz}, \mathrm{CDCl}_{3}\right) \delta=170.7-170.2-169.5$ (C=O OAc), 160.0 $(\mathrm{C}=\mathrm{O}), 158.3(\mathrm{C}=\mathrm{O}), 143.9(\mathrm{~N}-\mathrm{CH}=\mathrm{C}-\mathrm{N}), 124.0(\mathrm{~N}-\mathrm{CH}=\mathrm{C}-\mathrm{N}), 100.6\left(\mathrm{C1}^{\prime}\right), 72.6\left(\mathrm{C3}^{\prime}\right), 72.0\left(\mathrm{C5}^{\prime}\right), 71.3(\mathrm{C} 2)$, 70.9 (C2'), 68.4 (C4'), $67.9\left(\mathrm{~N}-\mathrm{CH}_{2}-\mathrm{CH}_{2}-\mathrm{O}\right), 67.7(\mathrm{CH}) ; 61.9$ (C6a,b), $50.1\left(\mathrm{CH}_{2}\right.$ bridge), $39.1\left(\mathrm{CH}_{2}\right), 31.1$ ( $\mathrm{N}-\mathrm{CH}_{2}-\mathrm{CH}_{2}-\mathrm{O}$ ), $20.9\left(\mathrm{CH}_{3}\right)$; HRMS (ESI-TOF) $\mathrm{m} / z$ [M+3H] ${ }^{3+}$ Calcd for $\mathrm{C}_{172} \mathrm{H}_{227} \mathrm{~N}_{40} \mathrm{O}_{88}$ 1420.1500; found 1420.1522. 


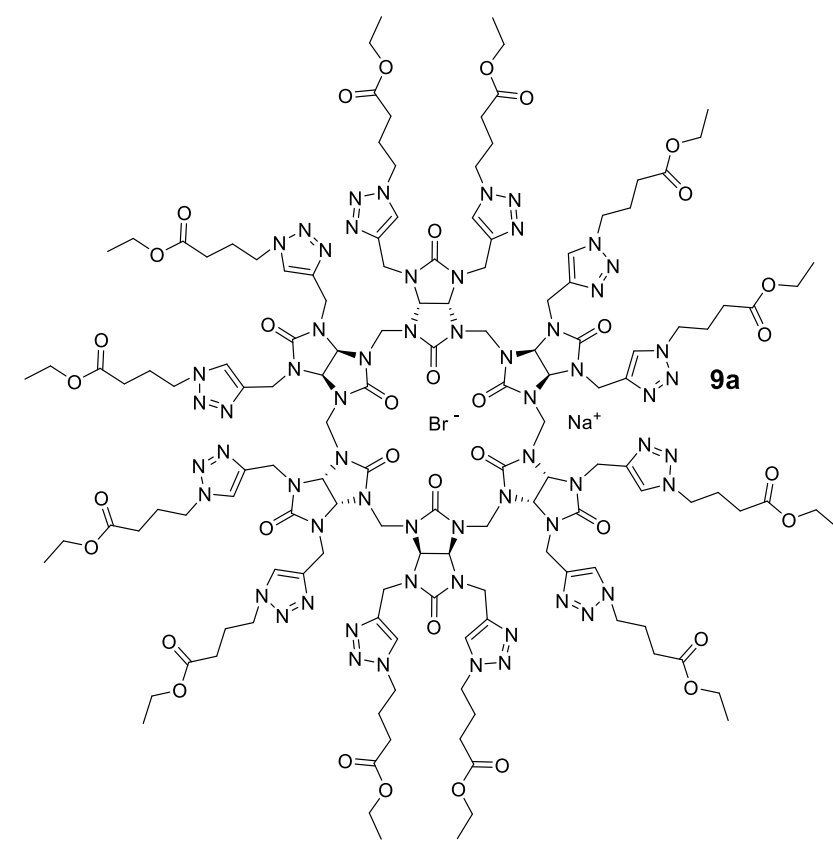

Sodium bromide @Dodecakis 1-((4-ethoxy-4-oxobutyl)-1H-1,2,3-triazol-4-yl)methyl) bambus[6]uril 9a To a solution of dodecapropargylbambus[6]uril tetra butyl bromide 3 (19.2 mg, $11.0 \mu \mathrm{mol}, 1.0 \mathrm{eq}$ ) and ethyl 4-azidobutanoate $7 \mathrm{a}^{3}(27.7 \mathrm{mg}, 0.18 \mathrm{mmol}, 16.0 \mathrm{eq})$ in DCE $(0.5 \mathrm{~mL})$, was added a solution of sodium ascorbate $1 \mathrm{M}$ in water $(44 \mu \mathrm{L}, 44.0 \mu \mathrm{mol}, 4.0 \mathrm{eq})$ and copper sulfate $1 \mathrm{M}$ in water $(22 \mu \mathrm{L}$, $22.0 \mu \mathrm{mol}, 2.0 \mathrm{eq})$ in $\mathrm{H}_{2} \mathrm{O} / \mathrm{t}-\mathrm{BuOH}(1 / 1.2 \mathrm{~mL})$. The reaction was subjected to microwave radiations for $2 \mathrm{~h}$ in a pressurized reactor, under magnetic stirring with $\mathrm{P}=50 \mathrm{~W}, \mathrm{~T}=80^{\circ} \mathrm{C}$. The mixture was concentrated under reduced pressure, diluted in a mixture of $\mathrm{MeCN} / \mathrm{H}_{2} \mathrm{O} / \mathrm{NH}_{4} \mathrm{OH}$ (9:1:1) and filtered with the same eluent $(25 \mathrm{~mL})$ on a small pad of $\mathrm{SiO}_{2}$. The filtrate was evaporated under reduced pressure and then purified by silicagel column chromatography $\left(\mathrm{CH}_{2} \mathrm{Cl}_{2} / \mathrm{MeOH}(9 / 1)\right)$ to afford $9 a$ (22.2 $\mathrm{mg}, 60 \%$ yield). IR ( $\left.\mathrm{v}_{\max } / \mathrm{cm}^{-1}\right)$ : 1703 (CO ester), 1697 (CO), $1476\left(\mathrm{CH}_{2}\right), 1214(\mathrm{CN}) ;{ }^{1} \mathrm{H} \mathrm{NMR}(400 \mathrm{MHz}$, $\left.\mathrm{CDCl}_{3}\right) \delta=7.65\left(12 \mathrm{H}\right.$, br s, N-CH=C-N), $5.85(12 \mathrm{H}, \mathrm{s}, \mathrm{CH}), 4.82-4.74\left(36 \mathrm{H}, \mathrm{m}, \mathrm{CH}_{2}\right.$ bridge, $\left.\mathrm{CH}_{2}\right), 4.35(24 \mathrm{H}$, $\left.\mathrm{m}, \mathrm{N}-\mathrm{CH}_{2}-\left(\mathrm{CH}_{2}\right)_{2}-\mathrm{C}=\mathrm{O}\right), 4.11\left(24 \mathrm{H}, \mathrm{q}, \mathrm{J}=7.0 \mathrm{~Hz}, \mathrm{O}-\mathrm{CH}_{2}-\mathrm{CH}_{3}\right), 2.33\left(24 \mathrm{H}, \mathrm{m}, \mathrm{N}-\left(\mathrm{CH}_{2}\right)_{2}-\mathrm{CH}_{2}-\mathrm{C}=\mathrm{O}\right), 2.17(24 \mathrm{H}$, m, N-CH $\left.-\mathrm{CH}_{2}-\mathrm{CH}_{2}-\mathrm{C}=\mathrm{O}\right), 1.23\left(36 \mathrm{H}, \mathrm{t}, \mathrm{J}=7.0 \mathrm{~Hz}, \mathrm{O}-\mathrm{CH}_{2}-\mathrm{CH}_{3}\right) ;{ }^{13} \mathrm{C} \mathrm{NMR}\left(100 \mathrm{MHz}, \mathrm{CDCl}_{3}\right) \delta=172.4(\mathrm{C}=\mathrm{O}$ ester), $159.6(\mathrm{C}=\mathrm{O}), 145.3(\mathrm{~N}-\mathrm{CH}=\mathrm{C}-\mathrm{N}), 123.4(\mathrm{~N}-\mathrm{CH}=\mathrm{C}-\mathrm{N}), 69.6(\mathrm{CH}), 60.8\left(\mathrm{O}-\mathrm{CH}_{2}-\mathrm{CH}_{3}\right), 52.4\left(\mathrm{CH}_{2}\right.$ bridge), $49.5\left(\mathrm{~N}-\mathrm{CH}_{2}-\left(\mathrm{CH}_{2}\right)_{2}-\mathrm{C}=\mathrm{O}\right), 39.4\left(\mathrm{CH}_{2}\right), 31.0\left(\mathrm{~N}-\left(\mathrm{CH}_{2}\right)_{2}-\mathrm{CH}_{2}-\mathrm{C}=\mathrm{O}\right), 25.5\left(\mathrm{~N}-\mathrm{CH}_{2}-\mathrm{CH}_{2}-\mathrm{CH}_{2}-\mathrm{C}=\mathrm{O}\right), 14.3$ (O- $\mathrm{CH}_{2}-\mathrm{CH}_{3}$ ); HRMS (ESI-TOF) m/z: [M-NaBr+3H] $]^{3+}$ Calcd for $\mathrm{C}_{138} \mathrm{H}_{195} \mathrm{~N}_{60} \mathrm{O}_{36}$ 1089.5085; found 1089.5098. 


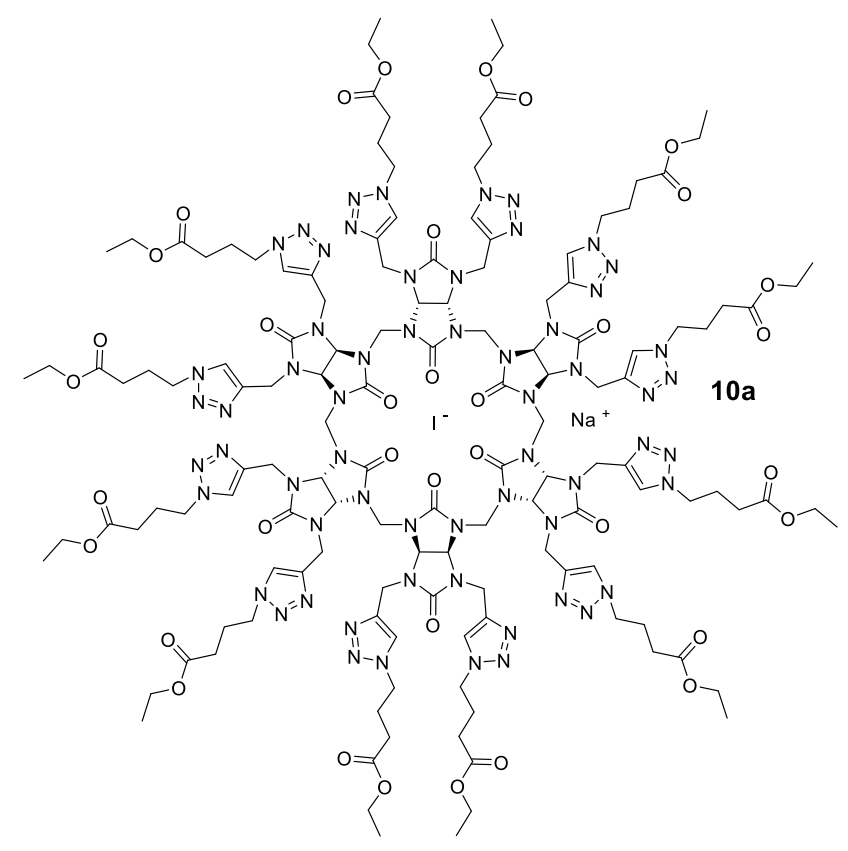

Sodium iodide @Dodecakis 1-((4-ethoxy-4-oxobutyl)-1H-1,2,3-triazol-4-yl)methyl)- bambus[6]uril 10a To a solution of dodecapropargylbambus[6]uril tetra butyl iodide 4 (30.0 mg, $17.0 \mu \mathrm{mol}, 1.0 \mathrm{eq}$ ) and ethyl 4-azidobutanoate $7 \mathrm{a}^{3}(43.0 \mathrm{mg}, 0.27 \mathrm{mmol}, 16.0 \mathrm{eq})$ in DCE $(0.5 \mathrm{~mL})$, was added a solution of sodium ascorbate $1 \mathrm{M}$ in water $(68 \mu \mathrm{L}, 68.0 \mu \mathrm{mol}, 4.0 \mathrm{eq})$ and copper sulfate $1 \mathrm{M}$ in water $(34 \mu \mathrm{L}$, $34.0 \mu \mathrm{mol}, 2.0 \mathrm{eq})$ in $\mathrm{H}_{2} \mathrm{O} / \mathrm{t}-\mathrm{BuOH}(1 / 1.2 \mathrm{~mL})$. The reaction was subjected to microwave radiations for $2 \mathrm{~h}$ in a pressurized reactor, under magnetic stirring with $\mathrm{P}=50 \mathrm{~W}, \mathrm{~T}=80{ }^{\circ} \mathrm{C}$. The mixture was concentrated under reduced pressure, diluted in a mixture of $\mathrm{MeCN} / \mathrm{H}_{2} \mathrm{O} / \mathrm{NH}_{4} \mathrm{OH}$ (9:1:1) and filtered with the same eluent $(25 \mathrm{~mL})$ on a small pad of $\mathrm{SiO}_{2}$. The filtrate was evaporated under reduced pressure and then purified by silicagel column chromatography $\left(\mathrm{CH}_{2} \mathrm{Cl}_{2} / \mathrm{MeOH}(9 / 1)\right)$ to afford 10a (37.8 mg, 65\% yield). IR ( $\left.\mathrm{v}_{\max } / \mathrm{cm}^{-1}\right): 1723$ (CO ester), 1703 (CO), $1477\left(\mathrm{CH}_{2}\right), 1216(\mathrm{CN}) ;{ }^{1} \mathrm{H}$ NMR (400 $\left.\mathrm{MHz}, \mathrm{CDCl}_{3}\right) \delta=7.66(12 \mathrm{H}, \mathrm{br} \mathrm{s}, \mathrm{N}-\mathrm{CH}=\mathrm{C}-\mathrm{N}), 5.89(12 \mathrm{H}, \mathrm{br} \mathrm{s}, \mathrm{CH}), 4.87-4.78\left(36 \mathrm{H}, \mathrm{m}, \mathrm{CH}_{2}\right.$ bridge, $\left.\mathrm{CH}_{2}\right)$, $4.36\left(24 \mathrm{H}, \mathrm{m}, \mathrm{N}-\mathrm{CH}_{2}-\left(\mathrm{CH}_{2}\right)_{2}-\mathrm{C}=\mathrm{O}\right), 4.11\left(24 \mathrm{H}, \mathrm{q}, \mathrm{J}=7.0 \mathrm{~Hz}, \mathrm{O}-\mathrm{CH}_{2}-\mathrm{CH}_{3}\right), 2.33\left(24 \mathrm{H}, \mathrm{m}, \mathrm{N}-\left(\mathrm{CH}_{2}\right)_{2}-\mathrm{CH}_{2}-\mathrm{C}=\mathrm{O}\right)$, $2.18\left(24 \mathrm{H}, \mathrm{m}, \mathrm{N}-\mathrm{CH}_{2}-\mathrm{CH}_{2}-\mathrm{CH}_{2}-\mathrm{C}=\mathrm{O}\right), 1.23\left(36 \mathrm{H}, \mathrm{t}, J=7.0 \mathrm{~Hz}, \mathrm{O}-\mathrm{CH}_{2}-\mathrm{CH}_{3}\right) ;{ }^{13} \mathrm{C} \mathrm{NMR}\left(100 \mathrm{MHz}, \mathrm{CDCl}_{3}\right) \delta=$ 172.4 ( $\mathrm{C}=\mathrm{O}$ ester), 160.1 (C=O), 159.3 (C=O), $145.8(\mathrm{~N}-\mathrm{CH}=\mathrm{C}-\mathrm{N}), 123.3(\mathrm{~N}-\mathrm{CH}=\mathrm{C}-\mathrm{N}), 69.7(\mathrm{CH}), 60.7$ (O$\left.\mathrm{CH}_{2}-\mathrm{CH}_{3}\right), 49.4\left(\mathrm{CH}_{2}\right.$ bridge), $47.5\left(\mathrm{~N}-\mathrm{CH}_{2}-\left(\mathrm{CH}_{2}\right)_{2}-\mathrm{C}=\mathrm{O}\right), 39.9\left(\mathrm{CH}_{2}\right), 30.9\left(\mathrm{~N}-\left(\mathrm{CH}_{2}\right)_{2}-\mathrm{CH}_{2}-\mathrm{C}=\mathrm{O}\right), 25.5\left(\mathrm{~N}-\mathrm{CH}_{2}-\right.$ $\left.\mathrm{CH}_{2}-\mathrm{CH}_{2}-\mathrm{C}=\mathrm{O}\right), 14.3\left(\mathrm{O}-\mathrm{CH}_{2}-\mathrm{CH}_{3}\right) ; \mathrm{HRMS}$ (ESI-TOF) m/z [M-Nal+2H] ${ }^{2+}$ Calcd for $\mathrm{C}_{138} \mathrm{H}_{194} \mathrm{~N}_{60} \mathrm{O}_{36} 1634.2605$; found 1634.2616 . 


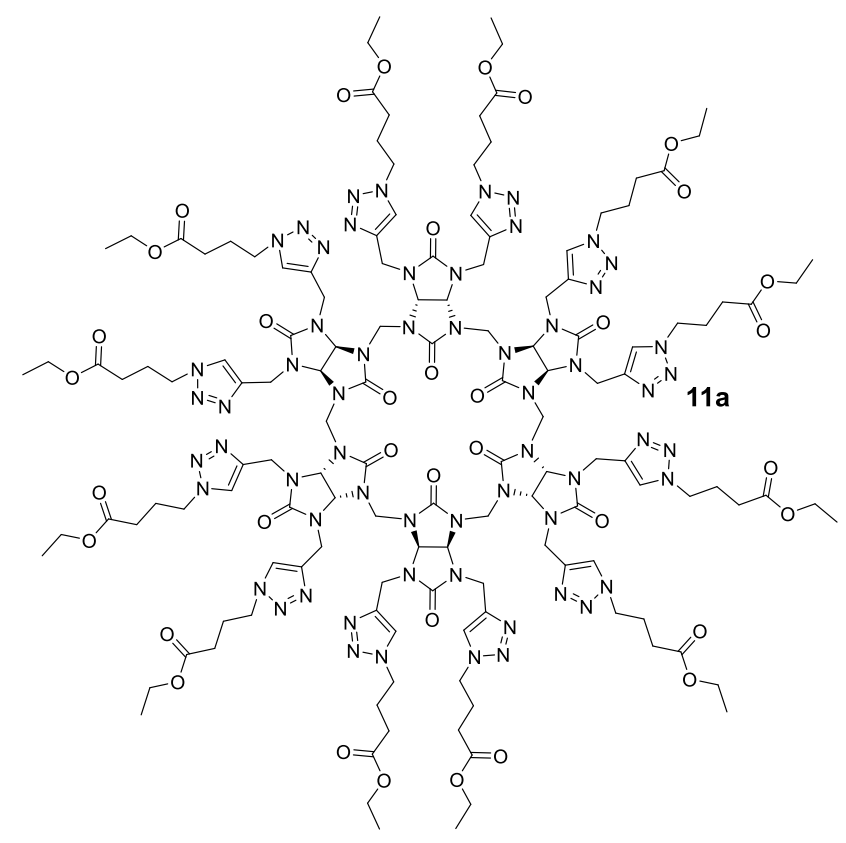

Dodecakis 1-((4-ethoxy-4-oxobutyl)-1H-1,2,3-triazol-4-yl)methyl) bambus[6]uril 11a To a solution of anion free dodecapropargylbambus[6]uril $6(35.4 \mathrm{mg}, 26.0 \mu \mathrm{mol}, 1 \mathrm{eq})$ and ethyl 4-azidobutanoate $7 \mathrm{a}^{3}$ (65.4 mg, $0.42 \mathrm{mmol}, 16 \mathrm{eq})$ in DMSO $(0.5 \mathrm{~mL})$, was added a solution of sodium ascorbate $1 \mathrm{M}$ in water (100 $\mu \mathrm{L}, 0.10 \mathrm{mmol}, 4 \mathrm{eq})$ and copper sulfate $1 \mathrm{M}$ in water $\left(52 \mu \mathrm{L}, 52.0 \mu \mathrm{mol}, 2\right.$ eq) in $\mathrm{H}_{2} \mathrm{O} / \mathrm{t}-\mathrm{BuOH}$ $(1 / 1.2 \mathrm{~mL})$. The reaction was subjected to microwave radiations in a pressurized reactor for $2 \mathrm{~h}$ under magnetic stirring with $\mathrm{P}=50 \mathrm{~W}, \mathrm{~T}=80^{\circ} \mathrm{C}$. The mixture was concentrated under reduced pressure, diluted in a mixture of $\mathrm{MeCN} / \mathrm{H}_{2} \mathrm{O} / \mathrm{NH}_{4} \mathrm{OH}$ (9:1:1) and filtered with the same eluent (25 mL) on a small pad of $\mathrm{SiO}_{2}$. The filtrate was evaporated under reduced pressure and then purified by silicagel column chromatography $\left(\mathrm{CH}_{2} \mathrm{Cl}_{2} / \mathrm{MeOH}(9 / 1)\right)$ to afford 11a $\left(42.5 \mathrm{mg}, 50 \%\right.$ yield). IR $\left(\mathrm{V}_{\max } / \mathrm{cm}^{-1}\right): 1703$ (CO ester), 1698 (CO), $1474\left(\mathrm{CH}_{2}\right), 1213(\mathrm{CN}) ;{ }^{1} \mathrm{H}$ NMR (400 MHz, $\left.\mathrm{CDCl}_{3}\right) \delta=7.52(12 \mathrm{H}, \mathrm{s}, \mathrm{N}-\mathrm{CH}=\mathrm{C}-\mathrm{N}), 5.79$ $(12 \mathrm{H}, \mathrm{s}, \mathrm{CH}), 4.98\left(12 \mathrm{H}, \mathrm{s}, \mathrm{CH}_{2}\right.$ bridge $), 4.64\left(24 \mathrm{H}, \mathrm{m}, \mathrm{CH}_{2}\right), 4.26\left(24 \mathrm{H}, \mathrm{m}, \mathrm{N}-\mathrm{CH}_{2}-\left(\mathrm{CH}_{2}\right)_{2}-\mathrm{C}=\mathrm{O}\right), 4.10(24 \mathrm{H}$, $\left.\mathrm{q}, \mathrm{J}=7.1 \mathrm{~Hz}, \mathrm{O}-\mathrm{CH}_{2}-\mathrm{CH}_{3}\right), 2.32\left(24 \mathrm{H}, \mathrm{t}, \mathrm{J}=6.7 \mathrm{~Hz}, \mathrm{~N}-\left(\mathrm{CH}_{2}\right)_{2}-\mathrm{CH}_{2}-\mathrm{C}=\mathrm{O}\right), 2.14\left(24 \mathrm{H}, \mathrm{m}, \mathrm{N}-\mathrm{CH}_{2}-\mathrm{CH}_{2}-\mathrm{CH}_{2}-\mathrm{C}=\mathrm{O}\right)$, $1.23\left(36 \mathrm{H}, \mathrm{t}, \mathrm{J}=7.1 \mathrm{~Hz}, \mathrm{O}-\mathrm{CH}_{2}-\mathrm{CH}_{3}\right) ;{ }^{13} \mathrm{C} \mathrm{NMR}\left(100 \mathrm{MHz}, \mathrm{CDCl}_{3}\right) \delta=172.4$ (C=O ester), 159.3 (C=O), 158.7 $(\mathrm{C}=\mathrm{O}), 145.4(\mathrm{~N}-\mathrm{CH}=\mathrm{C}-\mathrm{N}), 122.6(\mathrm{~N}-\mathrm{CH}=\mathrm{C}-\mathrm{N}), 68.5(\mathrm{CH}), 60.7\left(\mathrm{O}-\mathrm{CH}_{2}-\mathrm{CH}_{3}\right), 49.4\left(\mathrm{CH}_{2}\right.$ bridge $), 47.8(\mathrm{~N}-$

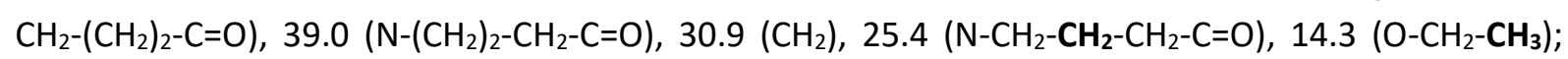
HRMS (ESI-TOF) $m / z[\mathrm{M}+2 \mathrm{H}]^{2+}$ Calcd for $\mathrm{C}_{138} \mathrm{H}_{194} \mathrm{~N}_{60} \mathrm{O}_{36}$ 1633.7592; found 1633.7598 . 


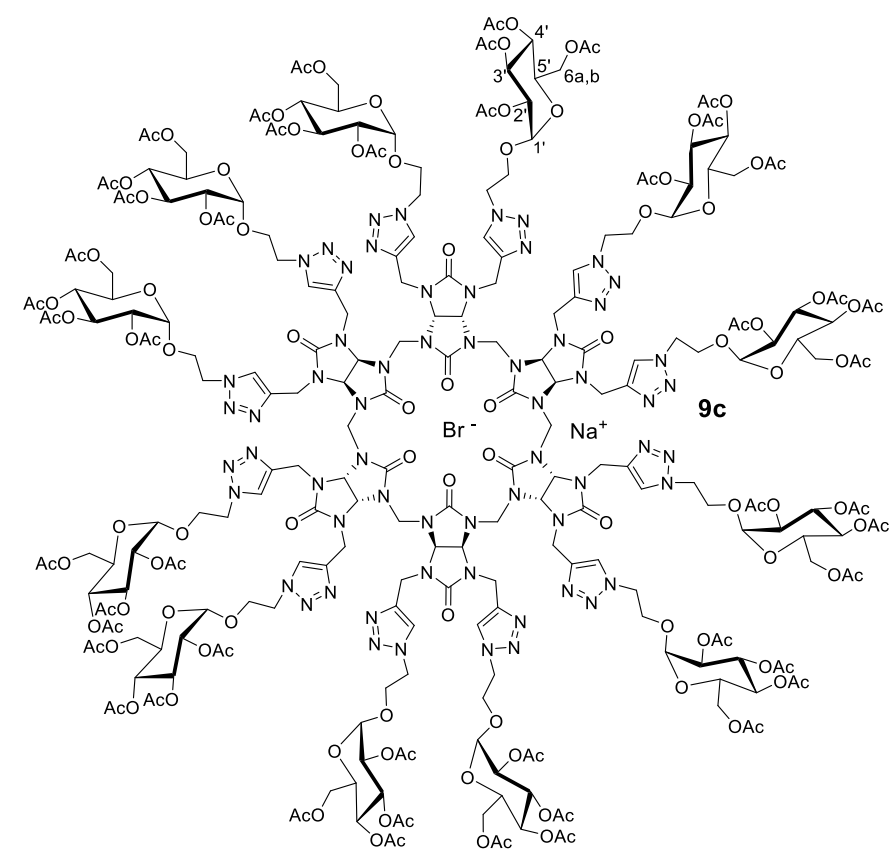

Sodium bromide @Dodecakis (1-(2-ethyl-2,3,4,6-tetra-O-acetyl- $\beta$-D-glucopyranoside)-1H-1,2,3triazol4-yl)methyl) bambus[6]uril 9c Sodium bromide @Dodecakis (1-(2-ethyl-2,3,4,6-tetra-0-acetylB-D-glucopyranoside)-1H-1,2,3-triazol4-yl)methyl) bambus[6]uril 9c To a solution of tetra ammonium bromide dodecapropargylbambus[6]uril 3 (20 mg, $11.74 \mu \mathrm{mol}, 1 \mathrm{eq}$ ) and 2-azidoethyl-2,3,4,6-tetra-Oacetyl- $\beta$-D-glucopyranoside $7 \mathrm{c}^{5}(78.4 \mathrm{mg}, 0.19 \mathrm{mmol}, 16 \mathrm{eq})$ in DCE $(0.5 \mathrm{~mL})$, was added a solution of sodium ascorbate $1 \mathrm{M}$ in water $(47 \mu \mathrm{L}, 47.0 \mu \mathrm{mol}, 4 \mathrm{eq})$ and copper sulfate $1 \mathrm{M}$ in water ( $23.5 \mu \mathrm{L}, 23.5$ $\mu \mathrm{mol}, 2 \mathrm{eq})$ in $\mathrm{H}_{2} \mathrm{O} / \mathrm{tBuOH}(1 / 1.2 \mathrm{~mL})$. The reaction was subjected to microwave radiations in a pressurized reactor for $2 \mathrm{~h}$ under magnetic stirring with $\mathrm{P}=50 \mathrm{~W}, \mathrm{~T}=80^{\circ} \mathrm{C}$. The mixture was concentrated under reduced pressure, diluted in a mixture of $\mathrm{MeCN} / \mathrm{H}_{2} \mathrm{O} / \mathrm{NH}_{4} \mathrm{OH}(9: 1: 1)$ and filtered with the same eluent $(25 \mathrm{~mL})$ on a small pad of $\mathrm{SiO}_{2}$. The filtrate was evaporated under reduced pressure and then purified by silicagel column chromatography $\left(\mathrm{CH}_{2} \mathrm{Cl}_{2} / \mathrm{MeOH}(9 / 1)\right)$ to afford $9 \mathrm{c}(54.4$ $\mathrm{mg}, 74 \%$ yield). [ $\alpha]_{D}-11.6$ (c 0.26 in $\left.\mathrm{CHCl}_{3}\right) ; \mathrm{IR}\left(\mathrm{v}_{\max } / \mathrm{cm}^{-1}\right): 1745$ (CO, OAc), 1700 (CO), $1477\left(\mathrm{CH}_{2}\right), 1211$ (CN), 1035 (CO); ${ }^{1} \mathrm{H}$ NMR (400 MHz, $\left.\mathrm{CDCl}_{3}\right) \delta=7.56(12 \mathrm{H}, \mathrm{d}, J=20.0 \mathrm{~Hz}, \mathrm{~N}-\mathrm{CH}=\mathrm{C}-\mathrm{N}), 5.79(12 \mathrm{H}, \mathrm{s}, \mathrm{CH})$, 5.20-5.11 (12H, m, H3'), $5.05\left(12 \mathrm{H}, \mathrm{dd}, J=6.1,9.6 \mathrm{~Hz}, \mathrm{H} 4^{\prime}\right), 4.97-4.92\left(24 \mathrm{H}, \mathrm{m}, \mathrm{H2}{ }^{\prime}, \mathrm{CH}_{2}\right.$ bridge), 4.56 $\left(24 \mathrm{H}, \mathrm{m}, \mathrm{CH}_{2}\right), 4.44\left(12 \mathrm{H}, \mathrm{br} \mathrm{s}, \mathrm{H} 1^{\prime}\right), 4.26-3.93\left(72 \mathrm{H}, \mathrm{m}, \mathrm{N}-\mathrm{CH}_{2}-\mathrm{CH}_{2}-\mathrm{O}, \mathrm{N}-\mathrm{CH}_{2}-\mathrm{CH}_{2}-\mathrm{O}, \mathrm{H} 6_{a, b}\right), 3.74-3.72$ $\left(12 \mathrm{H}, \mathrm{m}, \mathrm{H}^{\prime}\right), 2.08-2.01-1.99-1.93\left(144 \mathrm{H}, \mathrm{m}, \mathrm{CH}_{3}\right) ;{ }^{13} \mathrm{C} \mathrm{NMR}\left(100 \mathrm{MHz}, \mathrm{CDCl}_{3}\right) \delta=170.8-107.3-169.6$ (C=O OAC), 159.8 (C=O), $144.0(\mathrm{~N}-\mathrm{CH}=\mathrm{C}-\mathrm{N}), 123.8(\mathrm{~N}-\mathrm{CH}=\mathrm{C}-\mathrm{N}), 100.7$ (C1'), 72.7 (C3'), 72.0 (C2'), 71.0

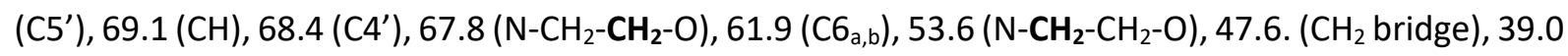
$\left(\mathrm{CH}_{2}\right)$, 20.9-20.7 $\left(\mathrm{CH}_{3}\right)$; HRMS (ESI-TOF) $\mathrm{m} / z[\mathrm{M}-\mathrm{NaBr}+4 \mathrm{H}]^{4+}$ Calcd for $\mathrm{C}_{258} \mathrm{H}_{340} \mathrm{~N}_{60} \mathrm{O}_{132}$ 1597.5429; found 1597.5464. 


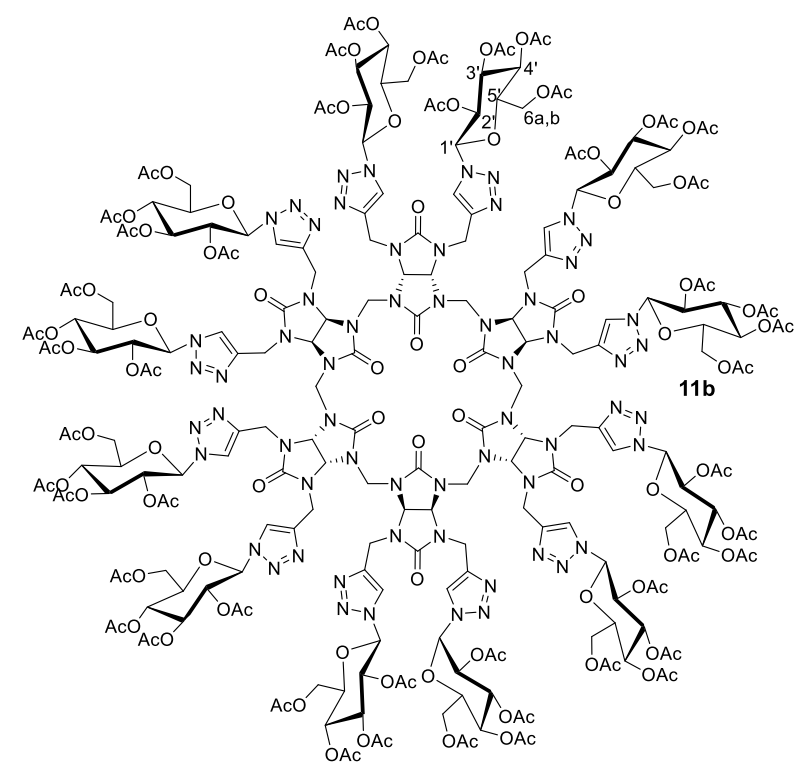

Dodecakis (1-(1- $\beta$-tetraacetylated glucose)-1H-1,2,3-triazol-4-yl)methyl)bambus[6]uril 11b To a solution of anion-free dodecapropargylbambus[6]uril $6(20.0 \mathrm{mg}, 14.5 \mu \mathrm{mol}, 1 \mathrm{eq})$ and 1- $\beta$-azido2,3,4,6 -tetra-O-acetyl-D-glucopyranoside $7 b^{4}(87.0 \mathrm{mg}, 0.23 \mathrm{mmol}, 16 \mathrm{eq})$ in DMSO $(0.5 \mathrm{~mL})$, was added a solution of was added a solution of sodium ascorbate $1 \mathrm{M}$ in water ( $58 \mu \mathrm{L}, 58.0 \mu \mathrm{mol}, 4 \mathrm{eq}$ ) and copper sulfate $1 \mathrm{M}$ in water ( $29 \mu \mathrm{L}, 29.0 \mu \mathrm{mol}, 2 \mathrm{eq})$ in $\mathrm{H}_{2} \mathrm{O} / \mathrm{tBuOH}(1 / 1.2 \mathrm{~mL})$. The reaction was subjected to microwave radiations in a pressurized reactor for $2 \mathrm{~h}$ under magnetic stirring with $\mathrm{P}=50$ $\mathrm{W}, \mathrm{T}=80{ }^{\circ} \mathrm{C}$. The mixture was concentrated under reduced pressure, diluted in a mixture of $\mathrm{MeCN} / \mathrm{H}_{2} \mathrm{O} / \mathrm{NH}_{4} \mathrm{OH}$ (9:1:1) and filtered with the same eluent $\left(25 \mathrm{~mL}\right.$ ) on a small pad of $\mathrm{SiO}_{2}$. The filtrate was evaporated under reduced pressure and then purified by silicagel column chromatography $\left(\mathrm{CH}_{2} \mathrm{Cl}_{2} / \mathrm{MeOH}(9 / 1)\right)$ to afford 11b (47.5 mg, 56\% yield). [ $\left.\alpha\right]_{D}-12.8$ (c 0.25 in $\left.\mathrm{CHCl}_{3}\right) ; \mathrm{IR}\left(\mathrm{v}_{\max } / \mathrm{cm}^{-1}\right)$ : 1753 (CO, OAC), 1703 (CO), $1478\left(\mathrm{CH}_{2}\right), 1216$ (CN), 1040 (CO); ${ }^{1} \mathrm{H} \mathrm{NMR}\left(400 \mathrm{MHz}, \mathrm{CDCl}_{3}\right) \delta=7.96-7.83$ $(12 \mathrm{H}$, br s, N-CH=C-N), 5.92-5.89 (24H, m, CH, H1'), 5.38-5.27 (36H, m, H2', H3', H4'), 4.86-4.76 (36H, $\mathrm{m}, \mathrm{CH}_{2}, \mathrm{CH}_{2}$ bridge), 4.25-4.20 (24H, m, H6, $\left.\mathrm{H}_{\mathrm{a}, \mathrm{b}}\right), 4.04\left(12 \mathrm{H}, \mathrm{m}, \mathrm{H} 5^{\prime}\right), 2.05-1.97\left(144 \mathrm{H}, \mathrm{m}, \mathrm{CH}_{3}\right) ;{ }^{13} \mathrm{C} \mathrm{NMR}$ $\left(100 \mathrm{MHz}, \mathrm{CDCl}_{3}\right) \delta=170.8-170.1-169.5$ ( $\mathrm{C}=\mathrm{O}$ OAc), $159.3(\mathrm{C}=\mathrm{O}), 158.7(\mathrm{C}=\mathrm{O}), 145.7$ ( $\left.\mathrm{N}-\mathrm{CH}=\mathrm{C}-\mathrm{N}\right), 122.6$ $\left.(\mathrm{N}-\mathrm{CH}=\mathrm{C}-\mathrm{N}), 85.2\left(\mathrm{C1}^{\prime}\right), 75.0\left(\mathrm{CS}^{\prime}\right), 73.1\left(\mathrm{C} 3^{\prime}\right), 72.2(\mathrm{C2})^{\prime}\right), 70.2\left(\mathrm{C4}^{\prime}\right), 67.8(\mathrm{CH}), 61.4\left(\mathrm{C6}_{\mathrm{a}, \mathrm{b}}\right), 47.8\left(\mathrm{CH}_{2}\right.$ bridge), $41.6\left(\mathrm{CH}_{2}\right), 20.7-20.2\left(\mathrm{CH}_{3}\right)$; HRMS (ESI-TOF) $\mathrm{m} / z[\mathrm{M}+3 \mathrm{H}]^{3+}$ Calcd for $\mathrm{C}_{234} \mathrm{H}_{291} \mathrm{~N}_{60} \mathrm{O}_{120} 1953.6165$; found 1953.6186 . 


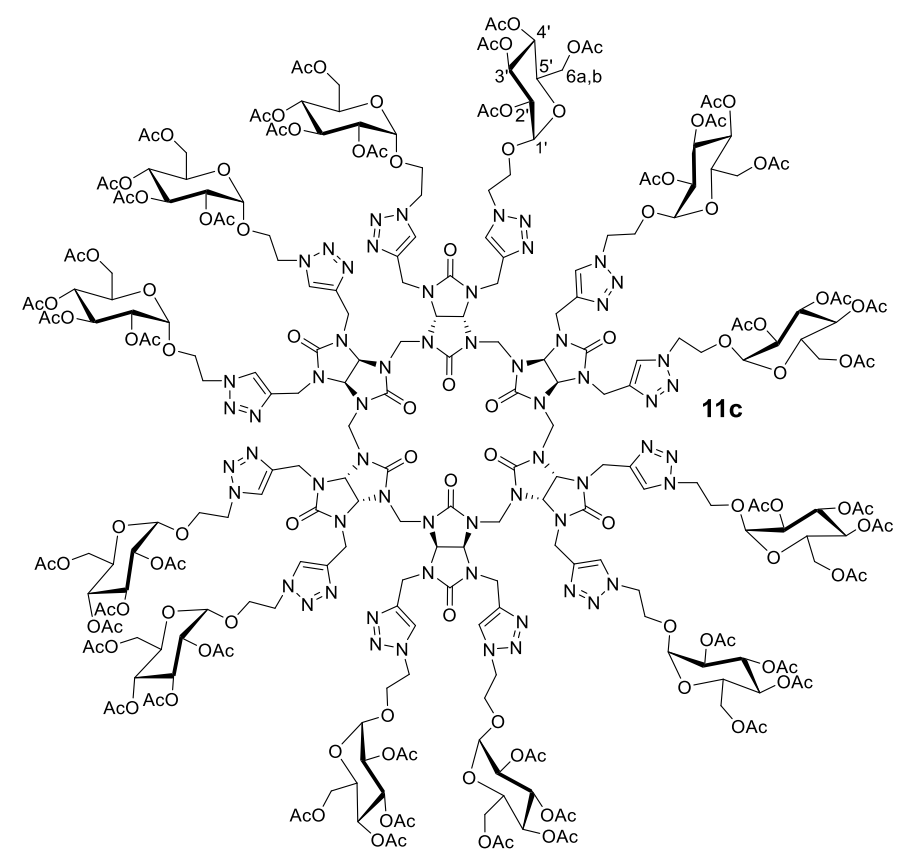

Dodecakis (1-(2-ethyl-2,3,4,6-tetra-O-acetyl- $\beta$-D-glucopyranoside)-1H-1,2,3-triazol4-yl)methyl) bambus[6]uril 11c To a solution of bambusuril $9 \mathrm{c}(32.2 \mathrm{mg}, 4.96 \mu \mathrm{mol}, 1 \mathrm{eq})$ in $\mathrm{MeOH}(1 \mathrm{~mL})$ was added $\operatorname{AgSbF}_{6}(2.9 \mathrm{mg}, 9.92 \mu \mathrm{mol}, 2 \mathrm{eq})$ and the mixture was centrifugated at $6500 \mathrm{rpm}$ for $5 \mathrm{~min}$. The solvent was discarded and the resulting precipitate was washed three times with $\mathrm{NH}_{4} \mathrm{OH}(2 \mathrm{~mL})$ to yield anionfree bambusuril 10c (29.5 mg, 93\% yield). IR ( $\left.\mathrm{v}_{\max } / \mathrm{cm}^{-1}\right): 1746$ (CO, OAc), 1699 (CO), $1478\left(\mathrm{CH}_{2}\right), 1210$ (CN), 1035 (CO); ${ }^{1} \mathrm{H}$ NMR (400 MHz, CDCl $\left.{ }_{3}\right) \delta=7.55(12 \mathrm{H}, \mathrm{d}, J=21.1 \mathrm{~Hz}, \mathrm{~N}-\mathrm{CH}=\mathrm{C}-\mathrm{N}), 5.79(12 \mathrm{H}, \mathrm{s}, \mathrm{CH})$, 5.20-5.13 (12H, m, H3'), $5.05\left(12 \mathrm{H}, \mathrm{dd}, J=6.1,9.6 \mathrm{~Hz}, \mathrm{H} 4^{\prime}\right), 4.97-4.92\left(24 \mathrm{H}, \mathrm{m}, \mathrm{H2}{ }^{\prime}, \mathrm{CH}_{2}\right.$ bridge), 4.58$4.55\left(24 \mathrm{H}, \mathrm{m}, \mathrm{CH}_{2}\right), 4.44\left(12 \mathrm{H}, \mathrm{sl}, \mathrm{H} 1^{\prime}\right), 4.33-3.93\left(72 \mathrm{H}, \mathrm{m}, \mathrm{N}-\mathrm{CH}_{2}-\mathrm{CH}_{2}-\mathrm{O}, \mathrm{N}-\mathrm{CH}_{2}-\mathrm{CH}_{2}-\mathrm{O}, \mathrm{H} \mathrm{a}_{\mathrm{a}, \mathrm{b}}\right), 3.75-3.72$ $\left(12 \mathrm{H}, \mathrm{m}, \mathrm{H} 5{ }^{\prime}\right), 2.08-2.01-1.99-1.93\left(144 \mathrm{H}, \mathrm{m}, \mathrm{CH}_{3}\right) ;{ }^{13} \mathrm{C} \mathrm{NMR}\left(100 \mathrm{MHz}, \mathrm{CDCl}_{3}\right) \delta=170.8-107.7-170.2-$ 169.5 (C=O OAC), 159.3 (C=O), 158.7 (C=O), $145.1(\mathrm{~N}-\mathrm{CH}=\mathrm{C}-\mathrm{N}), 123.5(\mathrm{~N}-\mathrm{CH}=\mathrm{C}-\mathrm{N}), 100.7$ (C1'), 72.7 (C3'), $72.0\left(\mathrm{C2}^{\prime}\right), 71.0\left(\mathrm{C5}^{\prime}\right), 68.4(\mathrm{CH}), 68.3\left(\mathrm{C}^{\prime}\right), 67.8\left(\mathrm{~N}-\mathrm{CH}_{2}-\mathrm{CH}_{2}-\mathrm{O}\right), 61.8\left(\mathrm{C6}_{\mathrm{a}, \mathrm{b}}\right), 49.9\left(\mathrm{~N}-\mathrm{CH}_{2}-\mathrm{CH}_{2}-\mathrm{O}\right)$, 47.8 $\left(\mathrm{CH}_{2}\right.$ bridge), $38.7\left(\mathrm{CH}_{2}\right), 20.9-20.7\left(\mathrm{CH}_{3}\right)$; HRMS (ESI-TOF) $\mathrm{m} / z[\mathrm{M}+4 \mathrm{H}]^{4+}$ Calcd for $\mathrm{C}_{258} \mathrm{H}_{340} \mathrm{~N}_{60} \mathrm{O}_{132}$ 1598.0443; found 1598.0465 .

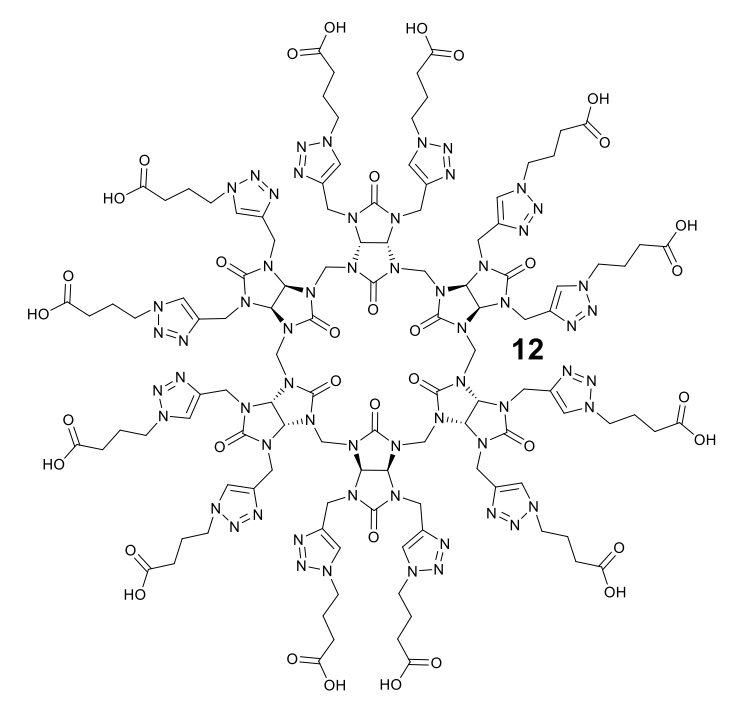


Dodecakis (1-(4 butanoic acid)-1H-1,2,3-triazol-4-yl)methyl) bambus[6]uril 12 To a solution of bambusuril 11a (27.3 mg, $8.4 \mu \mathrm{mol}, 1 \mathrm{eq})$ in THF/ $\mathrm{H}_{2} \mathrm{O}(1 / 1,2 \mathrm{~mL})$ at $0{ }^{\circ} \mathrm{C}$ was added LiOH $(8.5 \mathrm{mg}, 0.20$ mmol, $24 \mathrm{eq}$ ). The reaction was stirred at $0{ }^{\circ} \mathrm{C}$ for $5 \mathrm{~min}$ and then at $\mathrm{RT}$ for $24 \mathrm{~h}$. A solution of $\mathrm{HCl} 1 \mathrm{M}$ was added to reach $\mathrm{pH}=1$. EtOAc was added $(5 \mathrm{~mL})$ and the aqueous layer was separated. This step was repeated 2 times ( $2 \times 5 \mathrm{~mL}$ EtOAc). The aqueous layer was then lyophilized to give 12 (17.2 mg, $70 \%$ yield). IR $\left(\mathrm{v}_{\max } / \mathrm{cm}^{-1}\right): 3358.3(\mathrm{OH}), 1637.2(\mathrm{CO}), 1496.5\left(\mathrm{CH}_{2}\right), 1214.9(\mathrm{CN}) ;{ }^{1} \mathrm{H} \mathrm{NMR}(400 \mathrm{MHz}$, $\left.\mathrm{D}_{2} \mathrm{O}\right) \delta=7.94(12 \mathrm{H}, \mathrm{s}, \mathrm{N}-\mathrm{CH}=\mathrm{C}-\mathrm{N}), 5.73(12 \mathrm{H}, \mathrm{s}, \mathrm{CH}), 4.67-4.55\left(36 \mathrm{H}, \mathrm{m}, \mathrm{CH}_{2}\right.$ bridge, $\left.\mathrm{CH}_{2}\right), 4.40(24 \mathrm{H}, \mathrm{t}, \mathrm{J}$ $\left.=6.6 \mathrm{~Hz}, \mathrm{~N}-\mathrm{CH}_{2}-\left(\mathrm{CH}_{2}\right)_{2}-\mathrm{C}=\mathrm{O}\right) ; 2.24\left(24 \mathrm{H}, \mathrm{t}, \mathrm{J}=7.6 \mathrm{~Hz}, \mathrm{~N}-\left(\mathrm{CH}_{2}\right)_{2}-\mathrm{CH}_{2}-\mathrm{C}=0\right) ; 2.12\left(24 \mathrm{H}, \mathrm{m}, \mathrm{N}-\mathrm{CH}_{2}-\mathrm{CH}_{2}-\mathrm{CH}_{2}-\right.$ $\mathrm{C}=\mathrm{O}) ;{ }^{13} \mathrm{C}$ NMR (100 MHz, $\left.\mathrm{D}_{2} \mathrm{O}\right) \delta=178.0$ (C=O carboxylic acid), 160.1 (C=O), 144.7 ( N-CH=C-N), 123.5 ( $\mathrm{N}-\mathrm{CH}=\mathrm{C}-\mathrm{N}), 69.0(\mathrm{CH}), 49.6\left(\mathrm{CH}_{2}\right.$ bridge), $47.3\left(\mathrm{~N}-\mathrm{CH}_{2}-\left(\mathrm{CH}_{2}\right)_{2}-\mathrm{C}=\mathrm{O}\right), 39.4\left(\mathrm{CH}_{2}\right), 31.4\left(\mathrm{~N}-\left(\mathrm{CH}_{2}\right)_{2}-\mathrm{CH}_{2}-\mathrm{C}=\mathrm{O}\right)$, $25.2\left(\mathrm{~N}-\mathrm{CH}_{2}-\mathrm{CH}_{2}-\mathrm{CH}_{2}-\mathrm{C}=\mathrm{O}\right)$; HRMS (ESI-TOF) $\mathrm{m} / \mathrm{z}[\mathrm{M}+2 \mathrm{H}]^{2+}$ Calcd for $\mathrm{C}_{114} \mathrm{H}_{146} \mathrm{~N}_{60} \mathrm{O}_{36}$ 1465.5714; found 1465.5709.

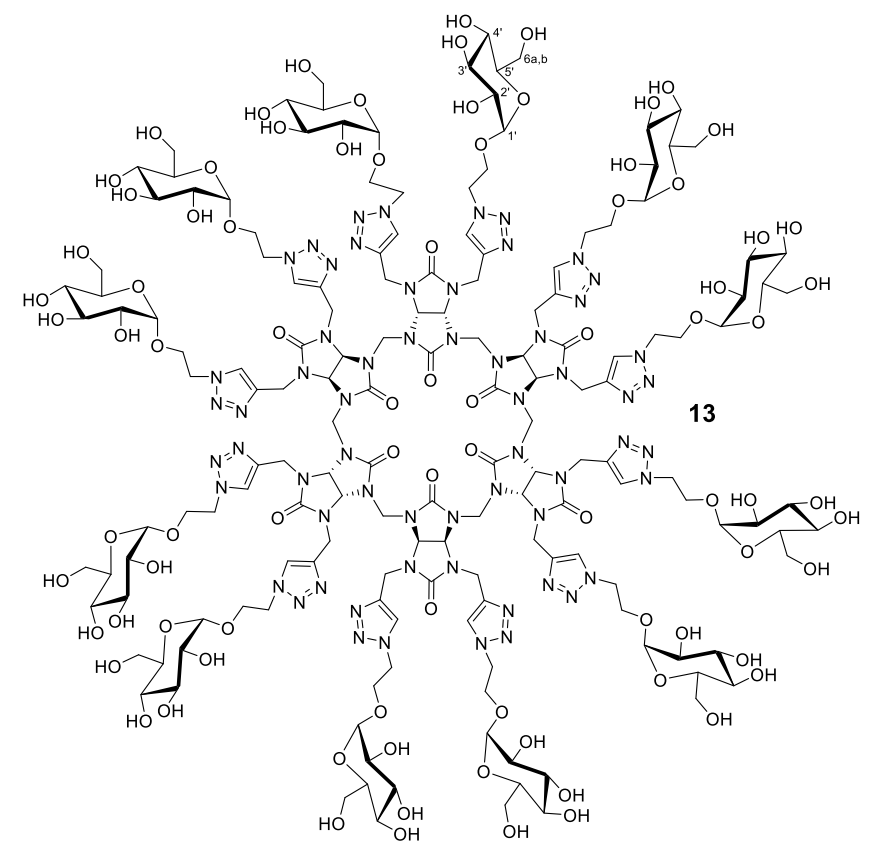

Dodecakis (1-(2-ethyl- $\beta$-D-glucopyranoside)-1H-1,2,3-triazol-4-yl)methyl) bambus[6]uril $\underline{13}$ Reaction of $9 \mathrm{c}$ with $\mathrm{AgSbF}_{6}$ yielded 11c (see above for details and datas of $9 \mathrm{c}$ ). To a solution of $11 \mathrm{c}(29.5 \mathrm{mg}$, $4.62 \mu \mathrm{mol}, 1 \mathrm{eq})$ in $\mathrm{H}_{2} \mathrm{O} / \mathrm{MeOH}\left(1.4 / 1.4 \mathrm{~mL}\right.$ ), was added amberlite IRN 78 ( $\left.\mathrm{HO}^{-}\right)(222 \mathrm{mg})$. The mixture was stirred $12 \mathrm{~h}$ at $40^{\circ} \mathrm{C}$. After filtration, and washing of the resin with water $(2 \mathrm{~mL})$ and methanol $(2$ $\mathrm{mL})$, the resulting filtrate was concentrated under reduced pressure and submitted to dialysis to afford 13 (17.4 mg, 86\% yield). [ $\alpha]_{\mathrm{D}}=-1.7$ (c 0.24 in water); IR $\left(\mathrm{v}_{\max } / \mathrm{cm}^{-1}\right): 3368(\mathrm{OH}), 1697(\mathrm{CO}), 1484\left(\mathrm{CH}_{2}\right)$, $1216(\mathrm{CN}), 1039(\mathrm{CO}) ;{ }^{1} \mathrm{H} N M R\left(400 \mathrm{MHz}, \mathrm{D}_{2} \mathrm{O}\right) \delta=7.99(12 \mathrm{H}, \mathrm{br} \mathrm{s}, \mathrm{N}-\mathrm{CH}=\mathrm{C}-\mathrm{N}), 5.76(12 \mathrm{H}, \mathrm{m}, \mathrm{CH}), 4.63-$ $4.46\left(36 \mathrm{H}, \mathrm{m}, 36 \mathrm{H}, \mathrm{H2} 2^{\prime}, \mathrm{H}^{\prime}, \mathrm{H}^{\prime}\right)$, 4.39-4.34 (12H, m, H1'), 4.19-4.16 (12H, m, $\left.\mathrm{CH}_{2}\right), 3.97-3.94(12 \mathrm{H}, \mathrm{m}$, $\left.\mathrm{CH}_{2}\right)$, 3.86-3.74 (12H, m, CH 2 bridge), 3.63-3.11 (84H, m, N- $\left.\mathrm{CH}_{2}-\mathrm{CH}_{2}-\mathrm{O}, \mathrm{N}-\mathrm{CH}_{2}-\mathrm{CH}_{2}-\mathrm{O}, \mathrm{H} 6 \mathrm{a}, \mathrm{b}, \mathrm{H} 5^{\prime}\right) ;{ }^{13} \mathrm{C}$ $\operatorname{NMR}\left(100 \mathrm{MHz}, \mathrm{D}_{2} \mathrm{O}\right) \delta=160.4(\mathrm{CO}), 159.4(\mathrm{CO}), 145.2(\mathrm{~N}-\mathrm{CH}=\mathrm{C}-\mathrm{N}), 125.2(\mathrm{~N}-\mathrm{CH}=\mathrm{C}-\mathrm{N}), 103.3\left(\mathrm{C1}^{\prime}\right), 76.6$ (C3'), $76.3\left(\mathrm{C2}^{\prime}\right), 73.6\left(\mathrm{C5}^{\prime}\right), 70.4\left(\mathrm{~N}-\mathrm{CH}_{2}-\mathrm{CH}_{2}-\mathrm{O}\right), 69.0\left(\mathrm{C} 4^{\prime}\right), 68.4(\mathrm{CH}), 61.5\left(\mathrm{CG}_{\mathrm{a}, \mathrm{b}}\right), 51.0\left(\mathrm{CH}_{2}\right.$ bridge), $48.3\left(\mathrm{~N}-\mathrm{CH}_{2}-\mathrm{CH}_{2}-\mathrm{O}\right)$, $39.4\left(\mathrm{CH}_{2}\right)$; HRMS (ESI-TOF) $\mathrm{m} / \mathrm{z}[\mathrm{M}+3 \mathrm{H}]^{3+}$ calcd for $\mathrm{C}_{162} \mathrm{H}_{243} \mathrm{~N}_{60} \mathrm{O}_{84} \mathrm{H}_{3}$ 1457.5524; found 1457.5515 . 


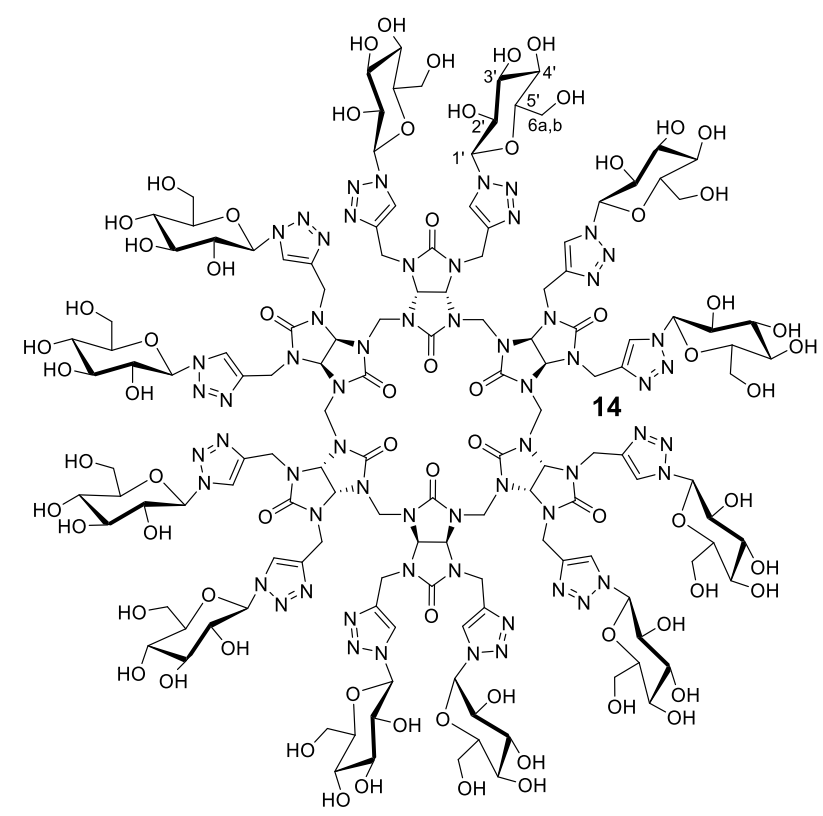

Dodecakis (1-(1- $\beta$-D-glucose)-1H-1,2,3-triazol-4-yl)methyl)bambus[6]uril $\underline{14}$ To a solution of $\mathbf{1 1 b}$ (20.0 mg, $3.4 \mu \mathrm{mol}, 1 \mathrm{eq})$ in $\mathrm{H}_{2} \mathrm{O} / \mathrm{MeOH}\left(1.0 / 1.0 \mathrm{~mL}\right.$ ), amberlite IRN 78 ( $\left.\mathrm{HO}^{-}\right)$(163.0 mg) was added. The suspension was stirred $12 \mathrm{~h}$ at $40^{\circ} \mathrm{C}$. After filtration, and washing of the resin with water $(2 \mathrm{~mL})$ and methanol $(2 \mathrm{~mL})$, the resulting filtrate was concentrated under reduced pressure and submitted to dialysis to afford 14 (11.9 mg, 91\% yield). [ $\alpha]_{D}=-3.0$ (c 0.26 in water); IR $\left(v_{\max } / \mathrm{cm}^{-1}\right): 3364(\mathrm{OH}), 2987$ $\left(\mathrm{CH}_{2}\right), 1692(\mathrm{CO}), 1485\left(\mathrm{CH}_{2}\right), 1217(\mathrm{CN}), 1056(\mathrm{CO}) ;{ }^{1} \mathrm{H}$ NMR $\left(400 \mathrm{MHz}, \mathrm{D}_{2} \mathrm{O}\right) \delta=8.18-8.10(12 \mathrm{H}, \mathrm{br} \mathrm{s}$, $\mathrm{N}-\mathrm{CH}=\mathrm{C}-\mathrm{N}), 5.81-5.62\left(24 \mathrm{H}, \mathrm{m}, \mathrm{CH}, \mathrm{H} 1^{\prime}\right), 4.68\left(12 \mathrm{H}, \mathrm{s}, \mathrm{CH}_{2}\right.$ bridge $), 3.95-3.83\left(24 \mathrm{H}, \mathrm{m}, \mathrm{CH}_{2}\right), 3.78-3.56$ $\left(72 \mathrm{H}, \mathrm{m}, \mathrm{H2}{ }^{\prime}, \mathrm{H} 3^{\prime}, \mathrm{H}^{\prime}, \mathrm{H}^{\prime}, \mathrm{H6}_{\mathrm{a}, \mathrm{b}}\right) ;{ }^{13} \mathrm{C} \mathrm{NMR}\left(100 \mathrm{MHz}, \mathrm{D}_{2} \mathrm{O}\right) \delta=159.3(\mathrm{C}=\mathrm{O}), 158.1(\mathrm{C}=\mathrm{O}), 143.8(\mathrm{~N}-$ $\mathrm{CH}=\mathrm{C}-\mathrm{N}), 122.3(\mathrm{~N}-\mathrm{CH}=\mathrm{C}-\mathrm{N}), 86.7\left(\mathrm{C1}^{\prime}\right), 77.9\left(\mathrm{C3}^{\prime}\right), 75.0\left(\mathrm{C}^{\prime}\right), 71.4\left(\mathrm{C2}^{\prime}\right), 71.1\left(\mathrm{C} 5^{\prime}\right), 67.9(\mathrm{CH}), 59.5$ $\left(\mathrm{C6}_{\mathrm{a}, \mathrm{b}}\right), 46.9\left(\mathrm{CH}_{2}\right.$ bridge), $37.7\left(\mathrm{CH}_{2}\right)$; HRMS (ESI-TOF) $\mathrm{m} / z[\mathrm{M}+3 \mathrm{H}]^{3+}$ Calcd for $\mathrm{C}_{138} \mathrm{H}_{195} \mathrm{~N}_{60} \mathrm{O}_{72} 1281.4462$; found 1281.4470 .

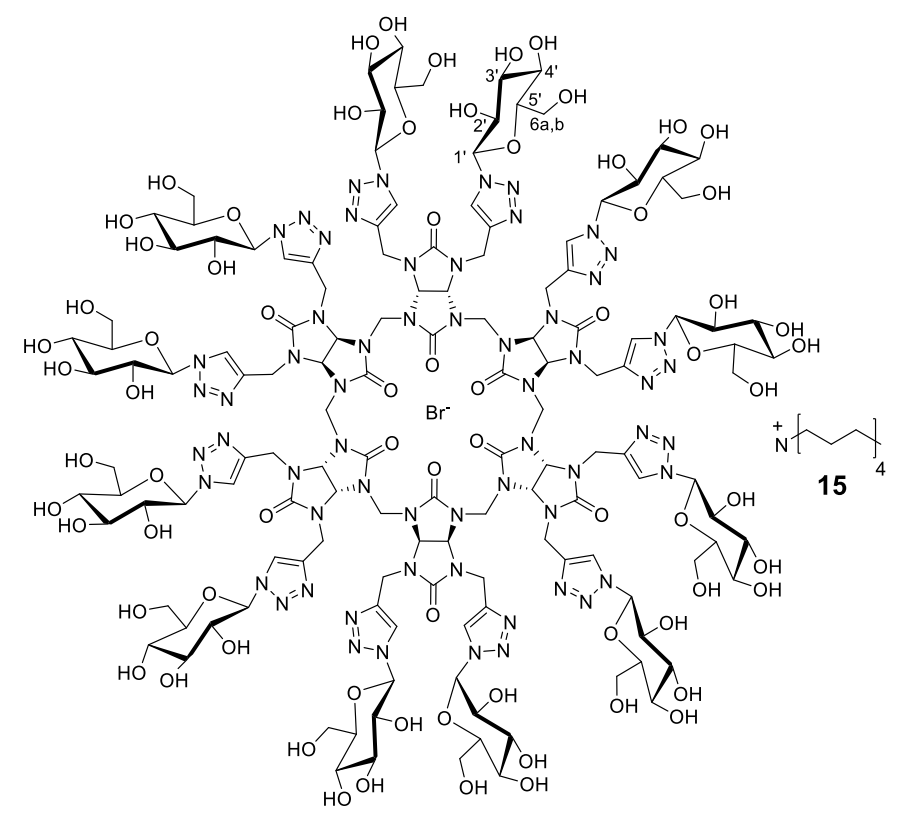

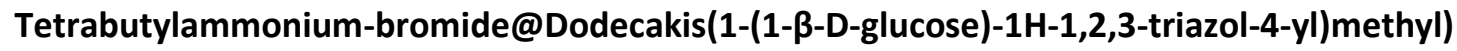

bambus[6]uril $\underline{15}$ To a solution of anion-free bambusuril $14(4.8 \mathrm{mg}, 1.24 \mu \mathrm{mol}, 1 \mathrm{eq})$ in water $(0.5 \mathrm{~mL})$ 
was added an aqueous solution of $\operatorname{TBABr}(19.1 \mathrm{mM} / \mathrm{L}, 63.3 \mu \mathrm{L}, 1.24 \mu \mathrm{mol}, 1 \mathrm{eq})$. After sonication for 5 min, the solution was concentrated to give bambusuril 15 (5.2 mg, quantitative yield). ${ }^{1} \mathrm{H}$ NMR (400 $\left.\mathrm{MHz}, \mathrm{D}_{2} \mathrm{O}\right) \delta=8.18(12 \mathrm{H}, \mathrm{s}, \mathrm{N}-\mathrm{CH}=\mathrm{C}-\mathrm{N}), 6.17-5.67\left(24 \mathrm{H}, \mathrm{m}, \mathrm{CH}, \mathrm{H} 1^{\prime}\right), 4.66\left(12 \mathrm{H}, \mathrm{s}, \mathrm{CH}_{2}\right.$ bridge), 3.91$3.59\left(96 \mathrm{H}, \mathrm{m}, \mathrm{CH}_{2}, \mathrm{H}^{\prime}, \mathrm{H}^{\prime}, \mathrm{H}^{\prime}, \mathrm{H}^{\prime}, \mathrm{H6}_{\mathrm{a}, \mathrm{b}}\right), 3.17-3.13\left(8 \mathrm{H}, \mathrm{m}, \mathrm{N}-\mathrm{CH}_{2}-\mathrm{CH}_{2}-\mathrm{CH}_{2}-\mathrm{CH}_{3}\right), 1.65-1.57(8 \mathrm{H}, \mathrm{m}$, $\left.\mathrm{N}-\mathrm{CH}_{2}-\mathrm{CH}_{2}-\mathrm{CH}_{2}-\mathrm{CH}_{3}\right), 1.44\left(8 \mathrm{H}, \mathrm{m}, \mathrm{N}-\mathrm{CH}_{2}-\mathrm{CH}_{2}-\mathrm{CH}_{2}-\mathrm{CH}_{3}\right), 0.98\left(12 \mathrm{H}, \mathrm{t}, \mathrm{J}=7.4 \mathrm{~Hz}, \mathrm{~N}-\mathrm{CH}_{2}-\mathrm{CH}_{2}-\mathrm{CH}_{2}-\mathrm{CH}_{3}\right)$; HRMS (ESI-TOF) $m / z$ [M-TBABr+3H] $]^{3+}$ Calcd for $\mathrm{C}_{138} \mathrm{H}_{195} \mathrm{~N}_{60} \mathrm{O}_{72} \mathrm{H}_{3}$ 1281.4462; found 1281.4470 .

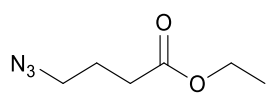

Ethyl 4-azidobutanoate $\underline{7 a}$ was prepared as reported. ${ }^{3}$

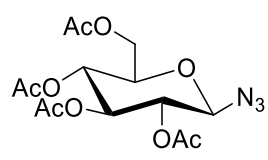

1- $\beta$-azido -2,3,4,6-O-acetyl-D-glucopyranoside $\underline{7 b}$ was prepared as reported. ${ }^{4}$

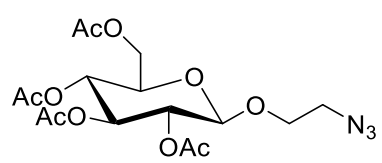

2-Azidoethyl-2,3,4,6-tetra-O-acetyl- $\beta$-D-glucopyranoside $\underline{7 c}$ was prepared as reported. ${ }^{5}$ 


\section{$\underline{{ }^{1} \mathrm{H} \text { and }{ }^{13} \mathrm{C} \text { NMR spectra }}$}

2,4-dipropargylglycoluril $\underline{1}\left({ }^{1} \mathrm{H}\right.$ NMR $400 \mathrm{MHz}$, DMSO-d6)
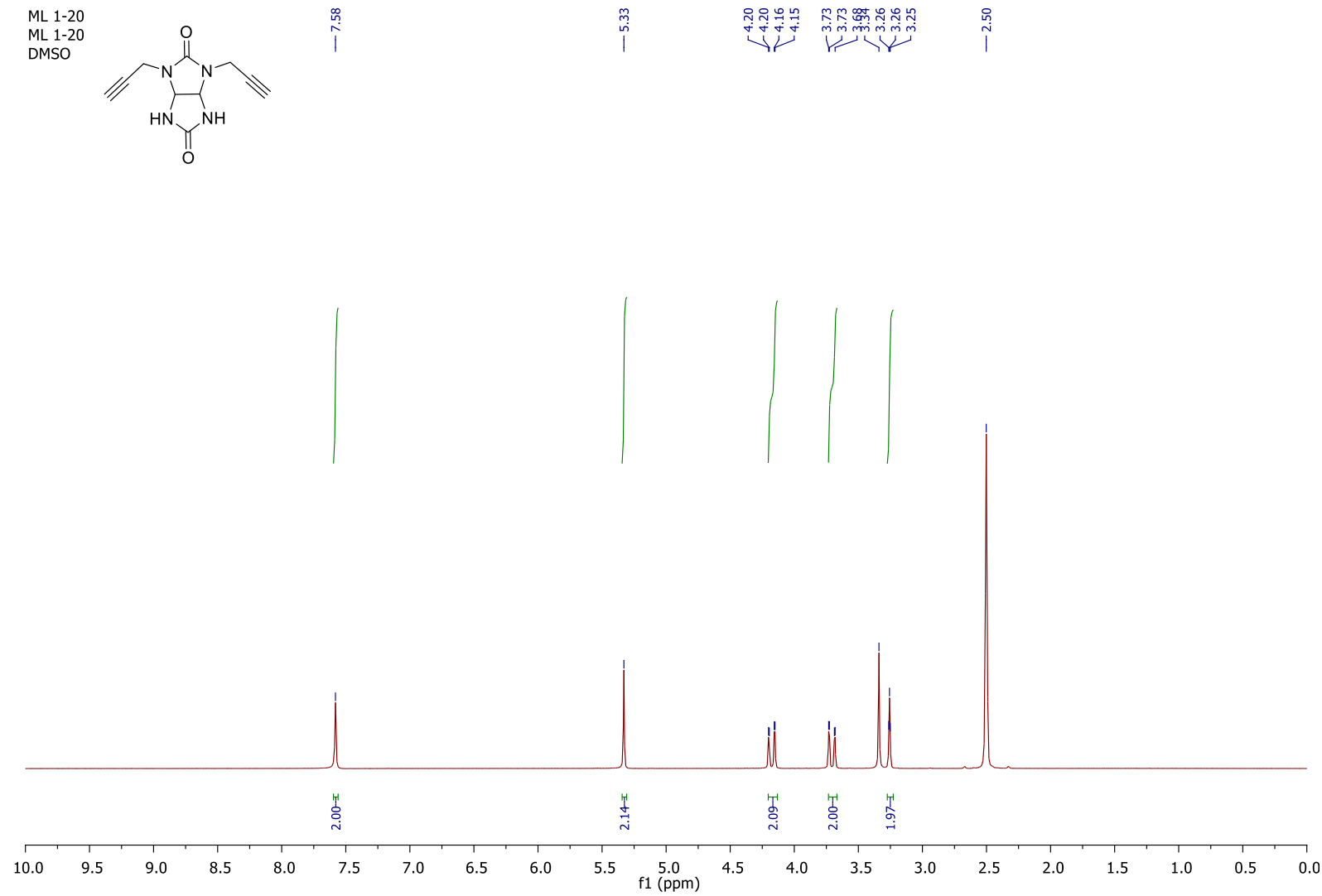

2,4-dipropargylglycoluril $\underline{1}\left({ }^{13} \mathrm{C}\right.$ NMR $100 \mathrm{MHz}$, DMSO-d6)

ML $1-20 \mathrm{C} 13$

ML $1-20 \mathrm{c13}$

节

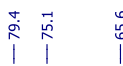

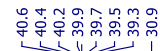


Octapropargylbambus[4] uril $\underline{2}\left({ }^{1} \mathrm{H}\right.$ NMR $400 \mathrm{MHz}$, DMSO-d6)

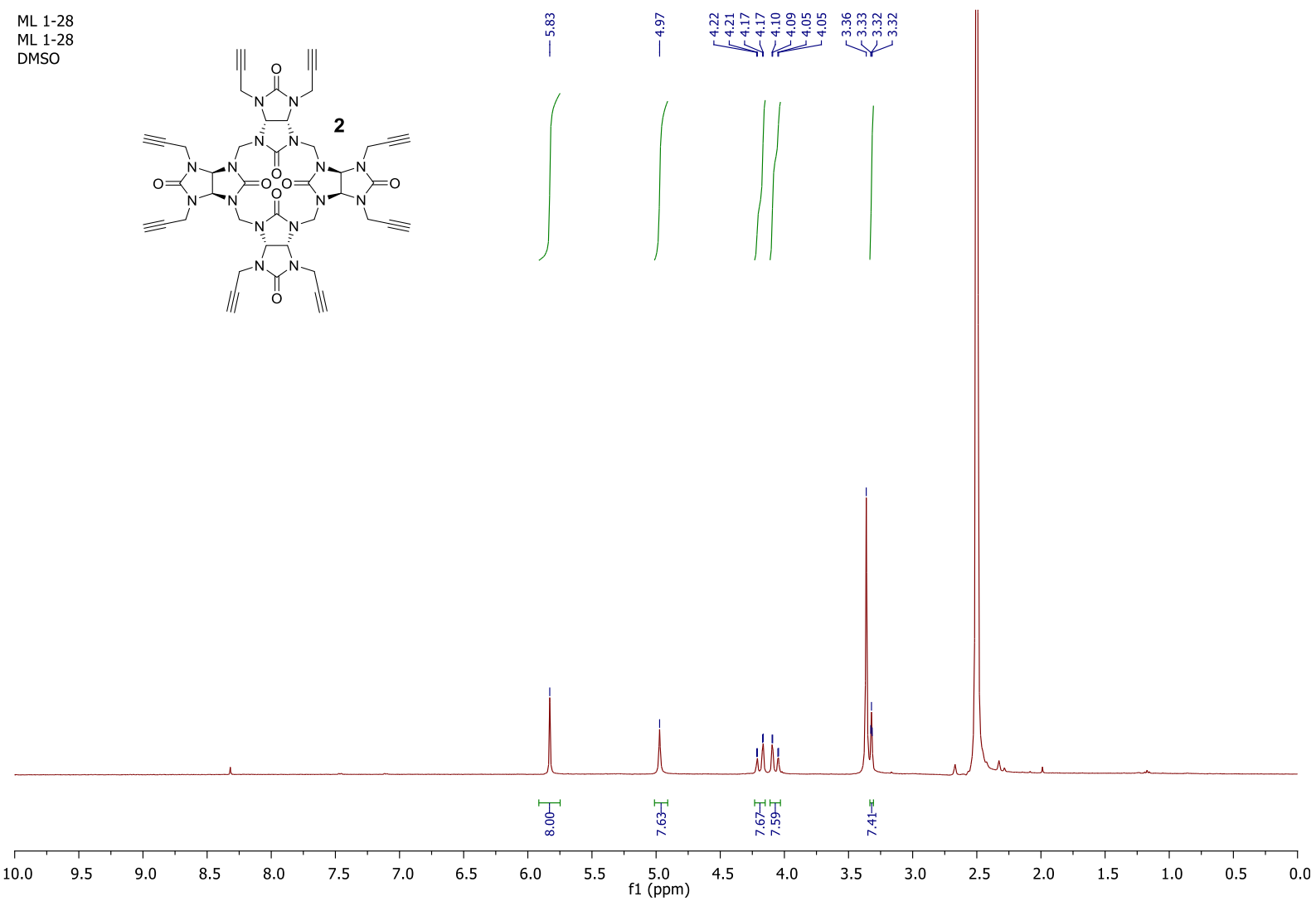

Octapropargylbambus[4]uril $\underline{2}\left({ }^{13} \mathrm{C}\right.$ NMR $100 \mathrm{MHz}$, Acetone-d6)

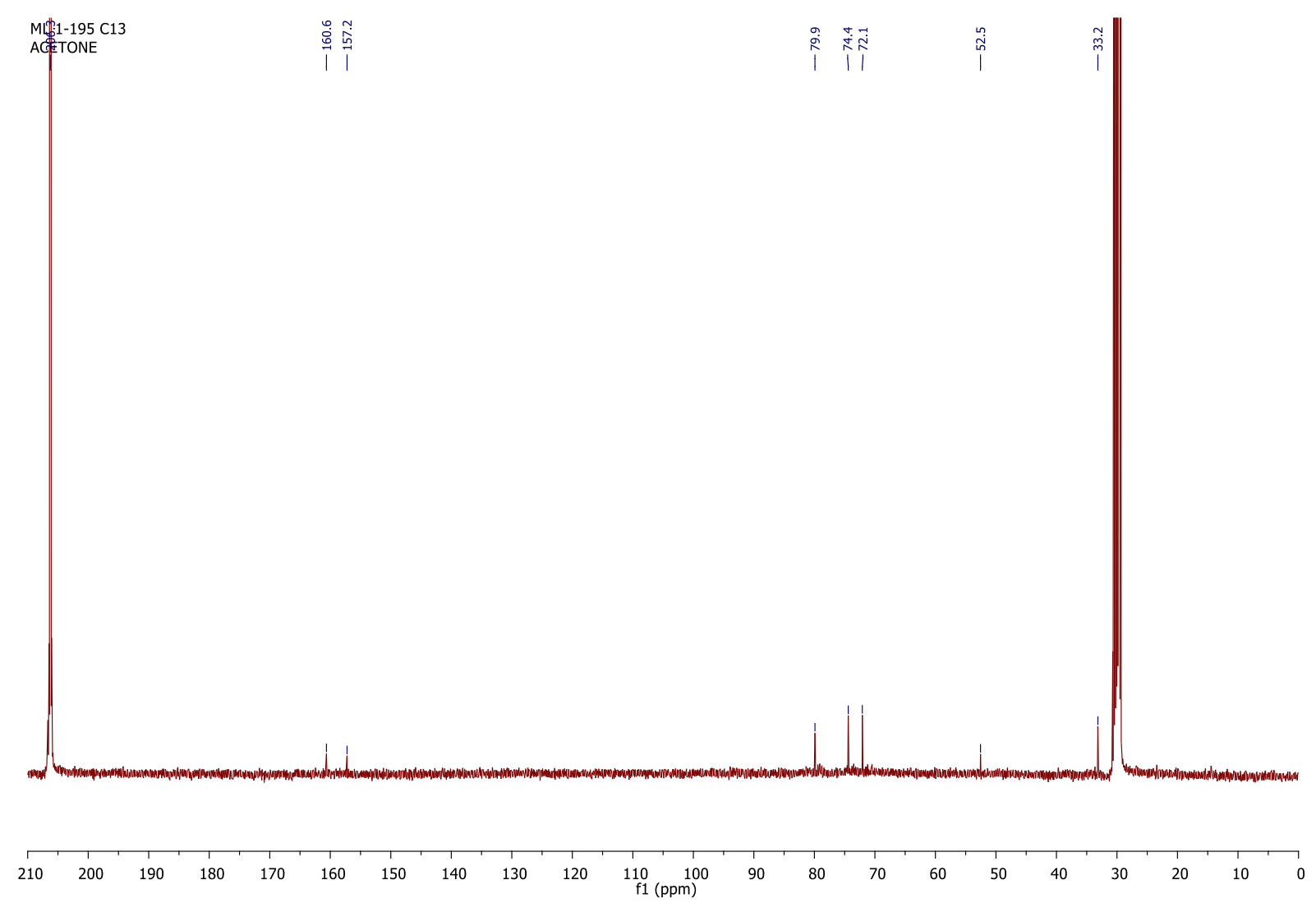


Tetrabutylammonium bromide @Dodecapropargylbambus[6]uril $\underline{3}\left({ }^{1} \mathrm{H}\right.$ NMR $400 \mathrm{MHz}$, Acetone-d6)

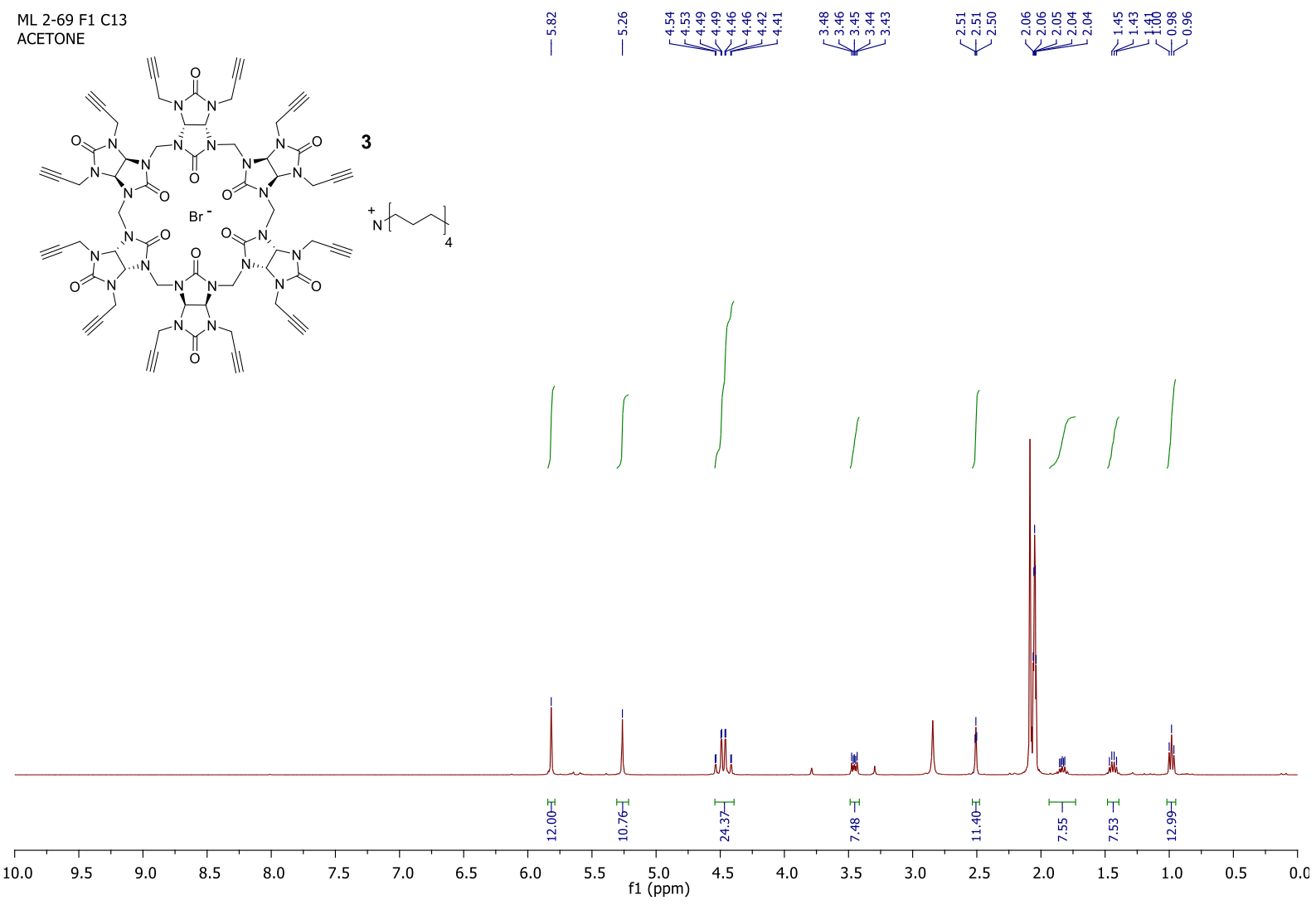

Tetrabutylammonium bromide @Dodecapropargylbambus[6]uril $\underline{3}\left({ }^{13} \mathrm{C} N \mathrm{NR} 100 \mathrm{MHz}\right.$, Acetone-d6)

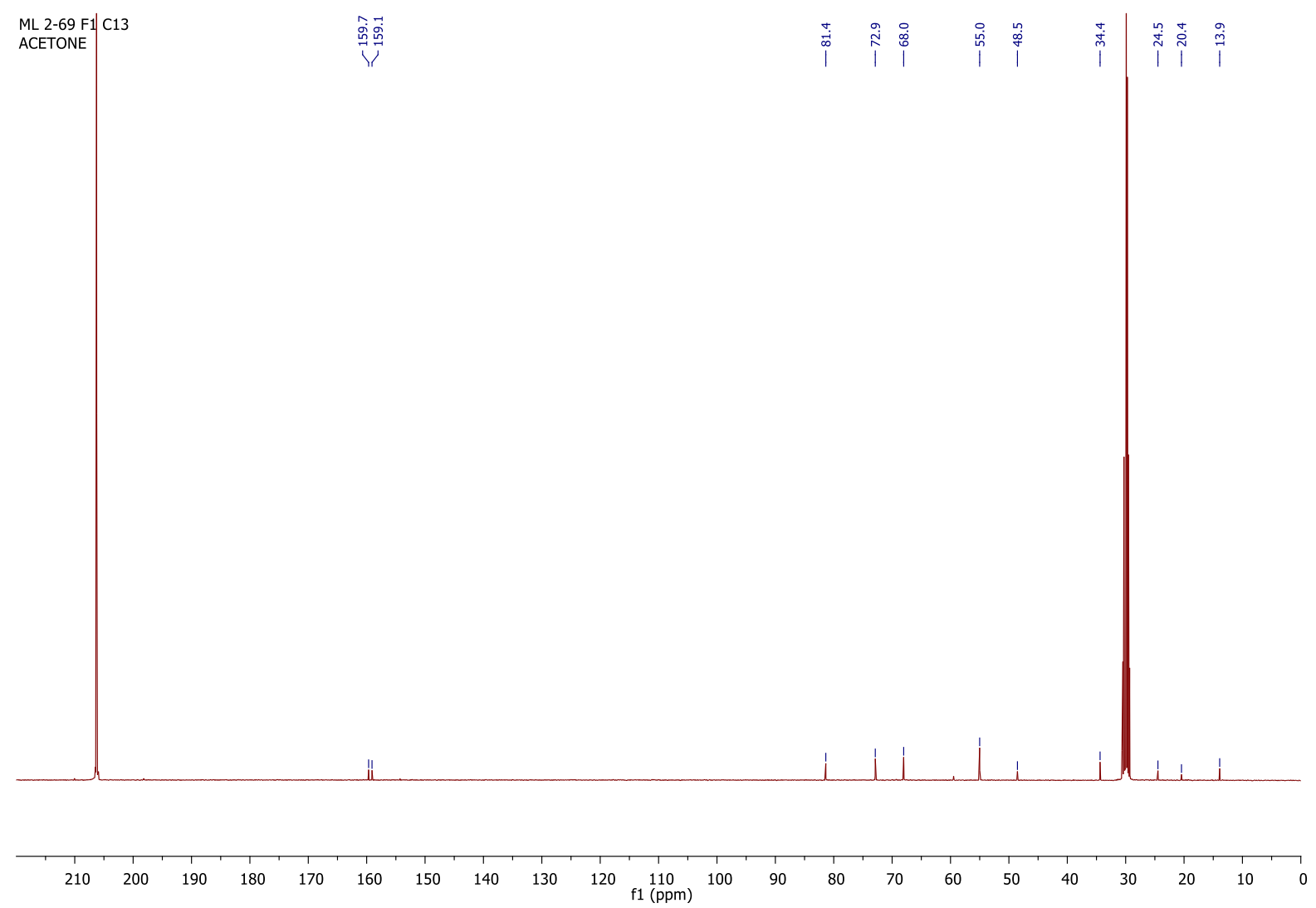


Tetrabutylammonium iodide @Dodecapropargylbambus[6]uril $\underline{4}\left({ }^{1} \mathrm{H} \mathrm{NMR} 400 \mathrm{MHz}\right.$, Acetone-d6)

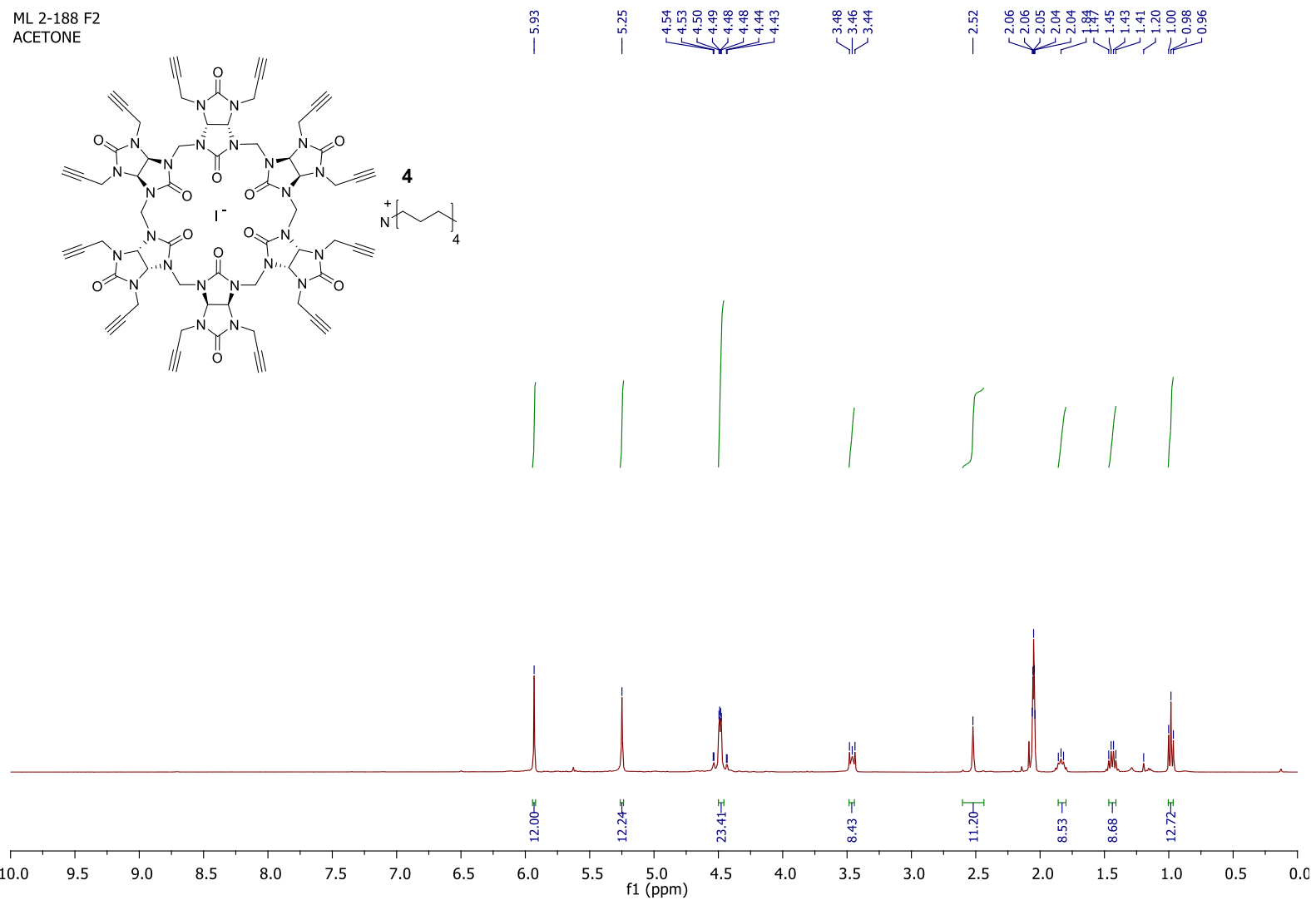

Tetrabutylammonium iodide @Dodecapropargylbambus[6]uril $\underline{4}\left({ }^{13} \mathrm{C} N \mathrm{NR} 100 \mathrm{MHz}\right.$, Acetone-d6)

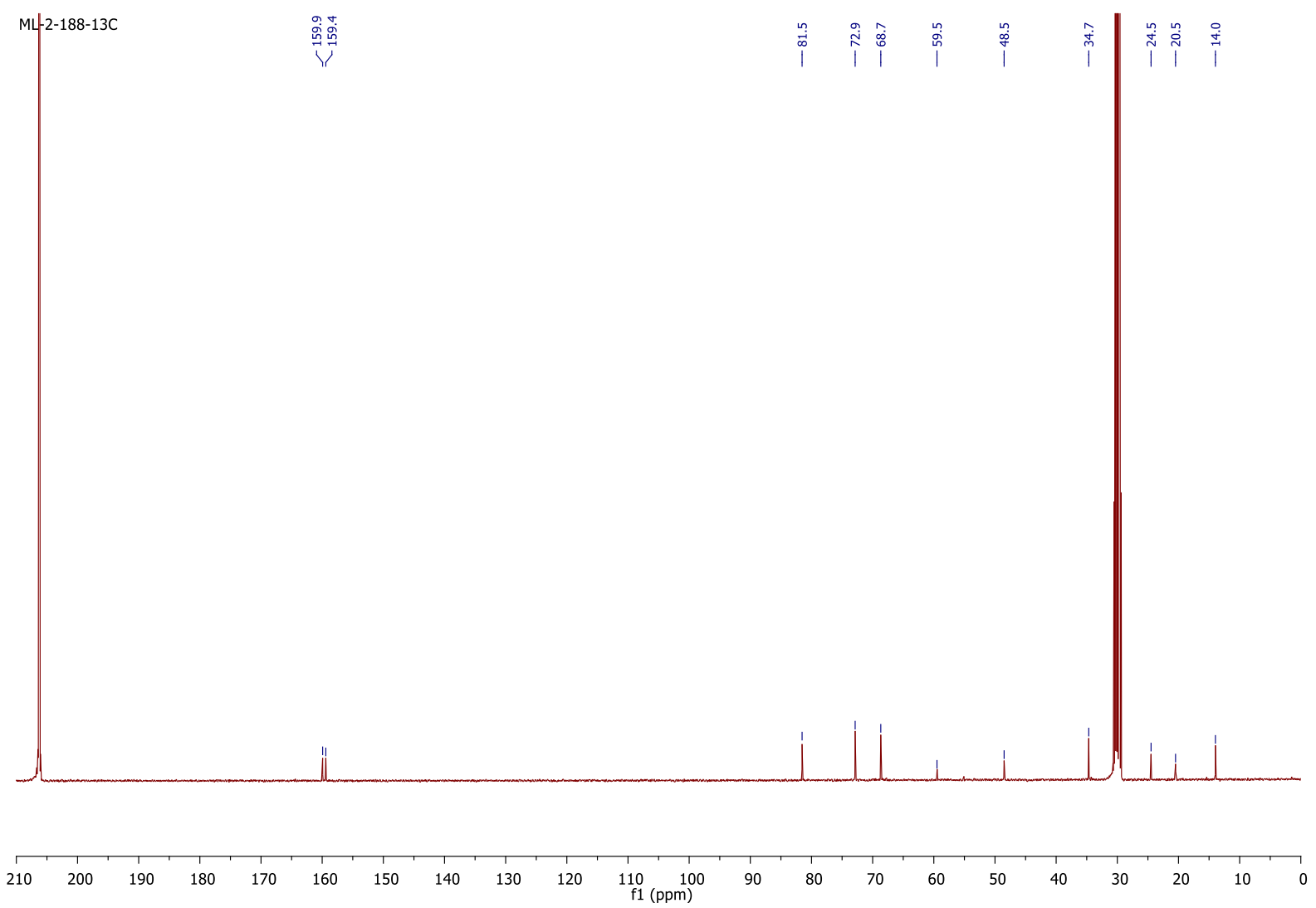


Tetrabutylammonium chloride @dodecapropargylbambus[6]uril $\underline{5}\left({ }^{1} \mathrm{H} N \mathrm{NR} 400 \mathrm{MHz}\right.$, Acetone-d6)
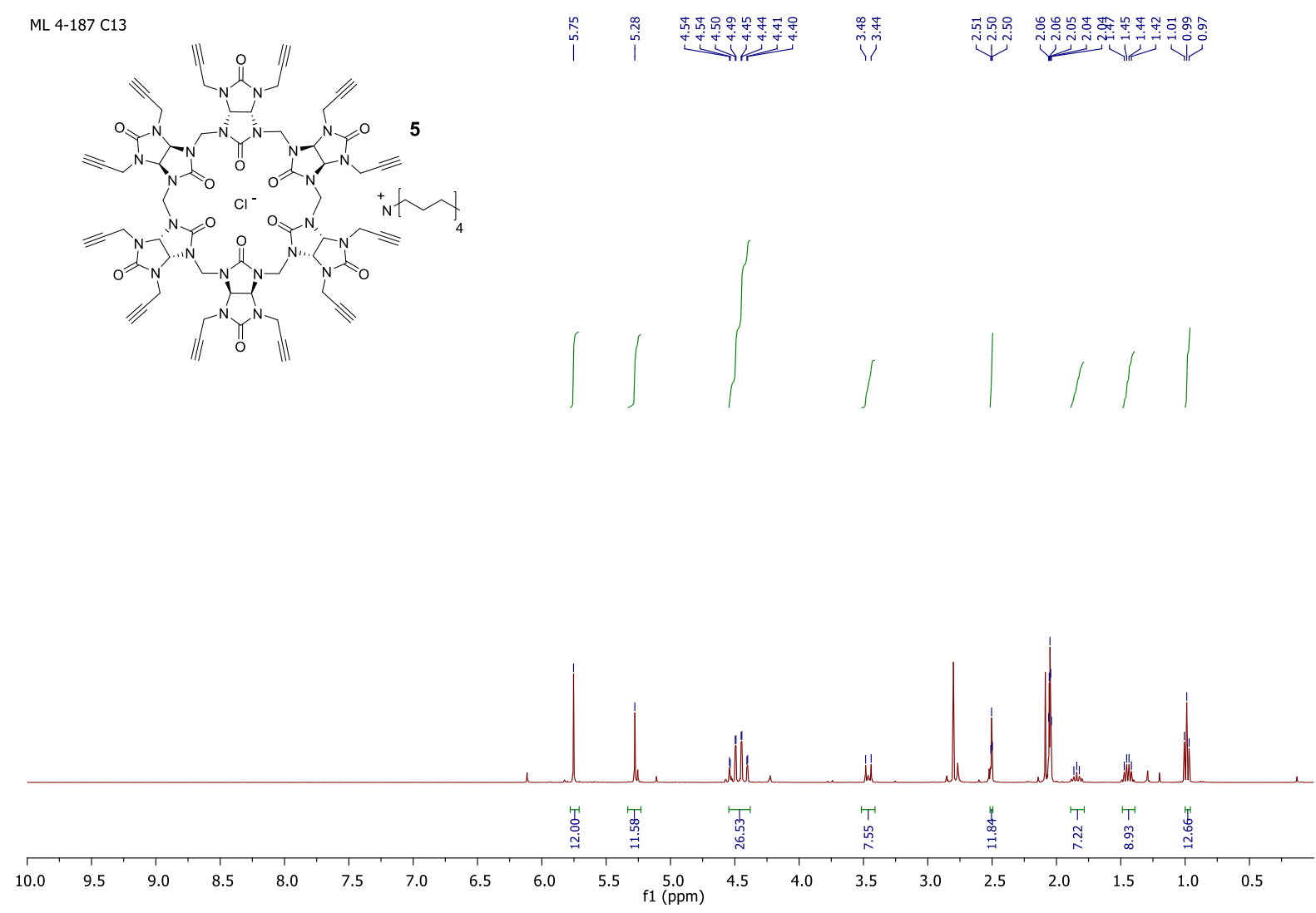

Tetrabutylammonium chloride @dodecapropargylbambus[6]uril $\underline{\mathbf{5}}\left({ }^{13} \mathrm{C} N \mathrm{NR} 100 \mathrm{MHz}\right.$, Acetone-d6)

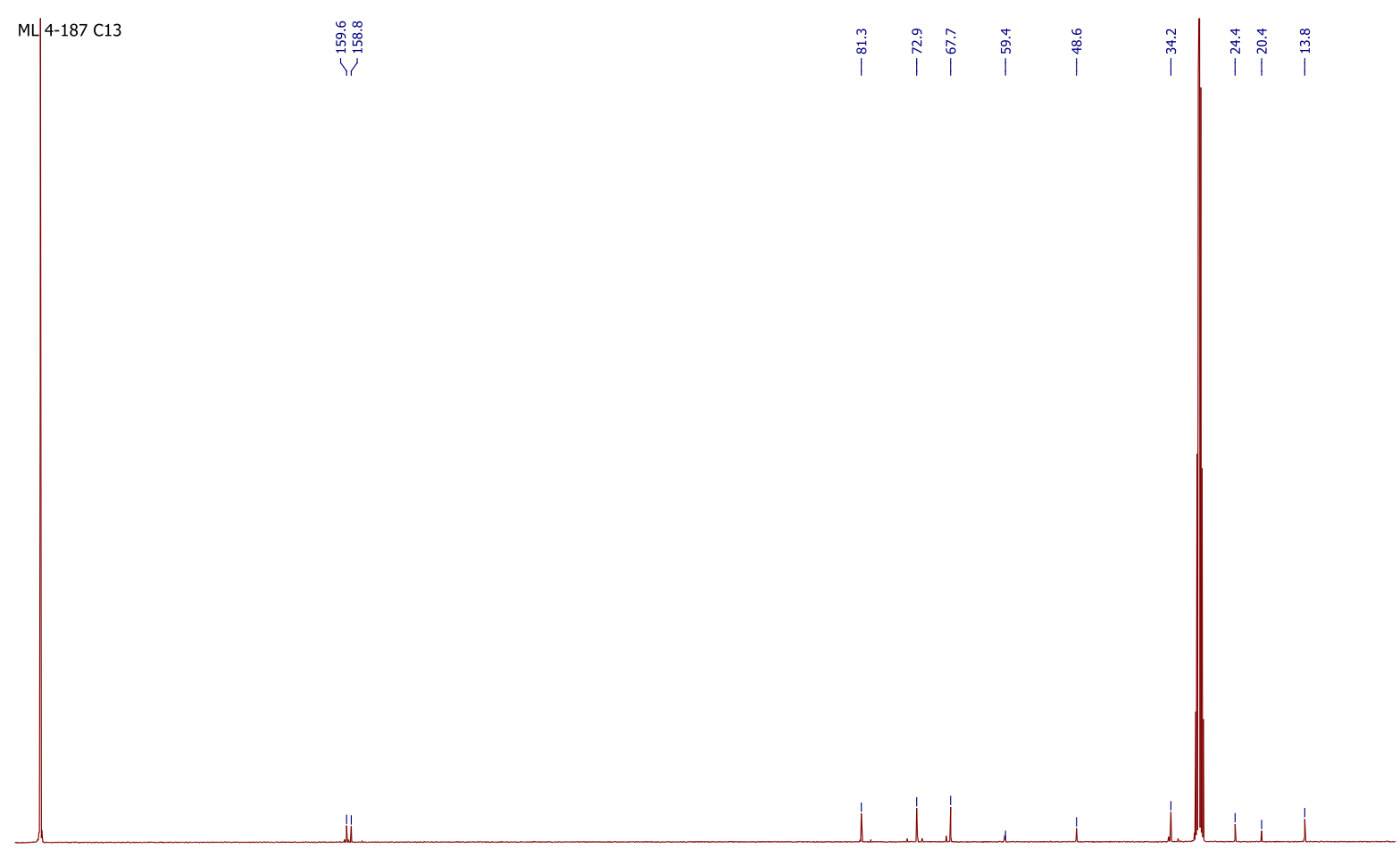

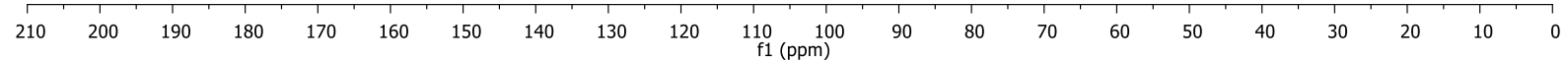


Dodecapropargylbambus[6]uril $\underline{6}\left({ }^{1} \mathrm{H}\right.$ NMR $400 \mathrm{MHz}$, DMSO-d6)

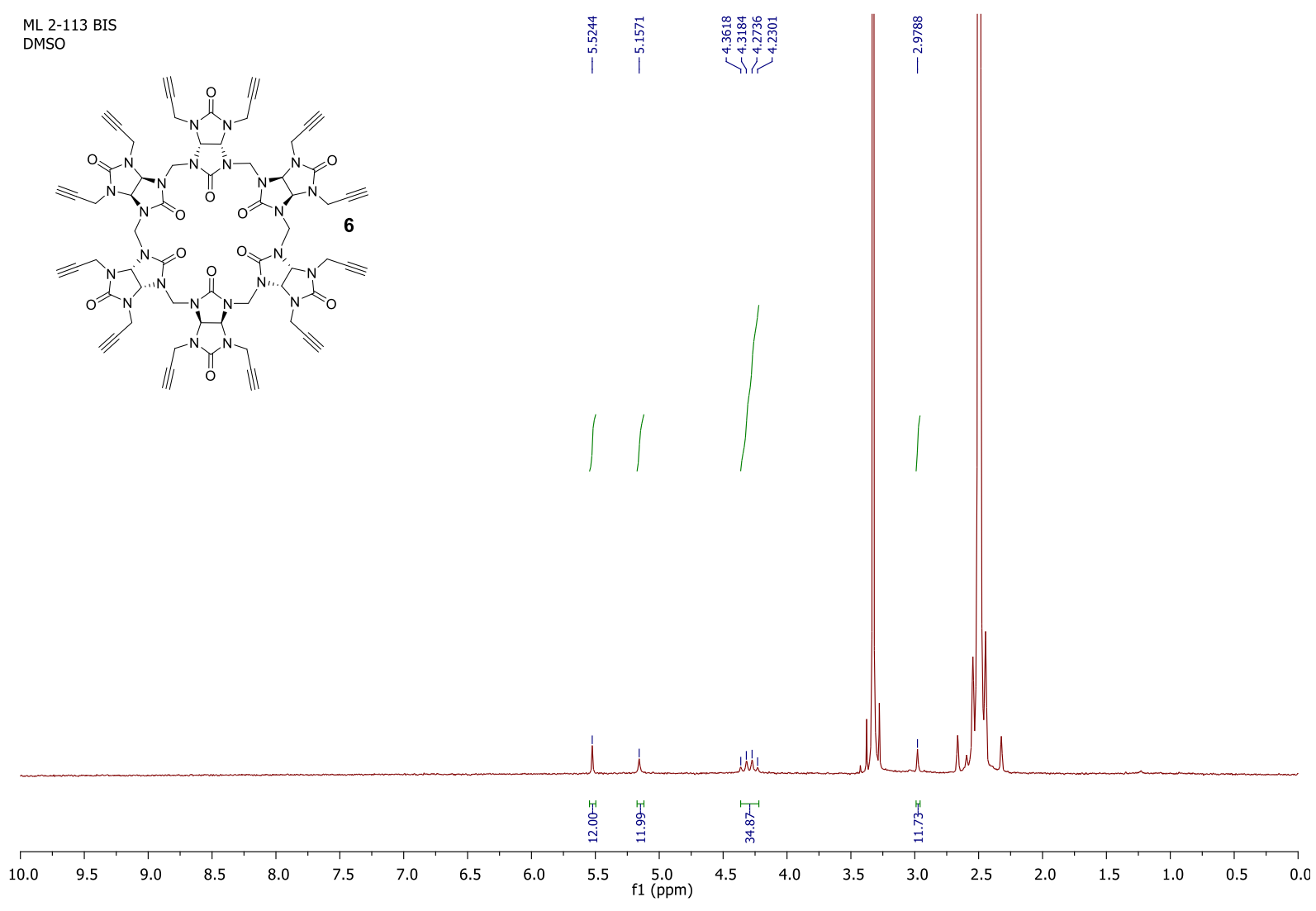

Dodecapropargylbambus[6]uril $\underline{6}\left({ }^{13} \mathrm{C}\right.$ NMR $100 \mathrm{MHz}$, Acetone-d6)

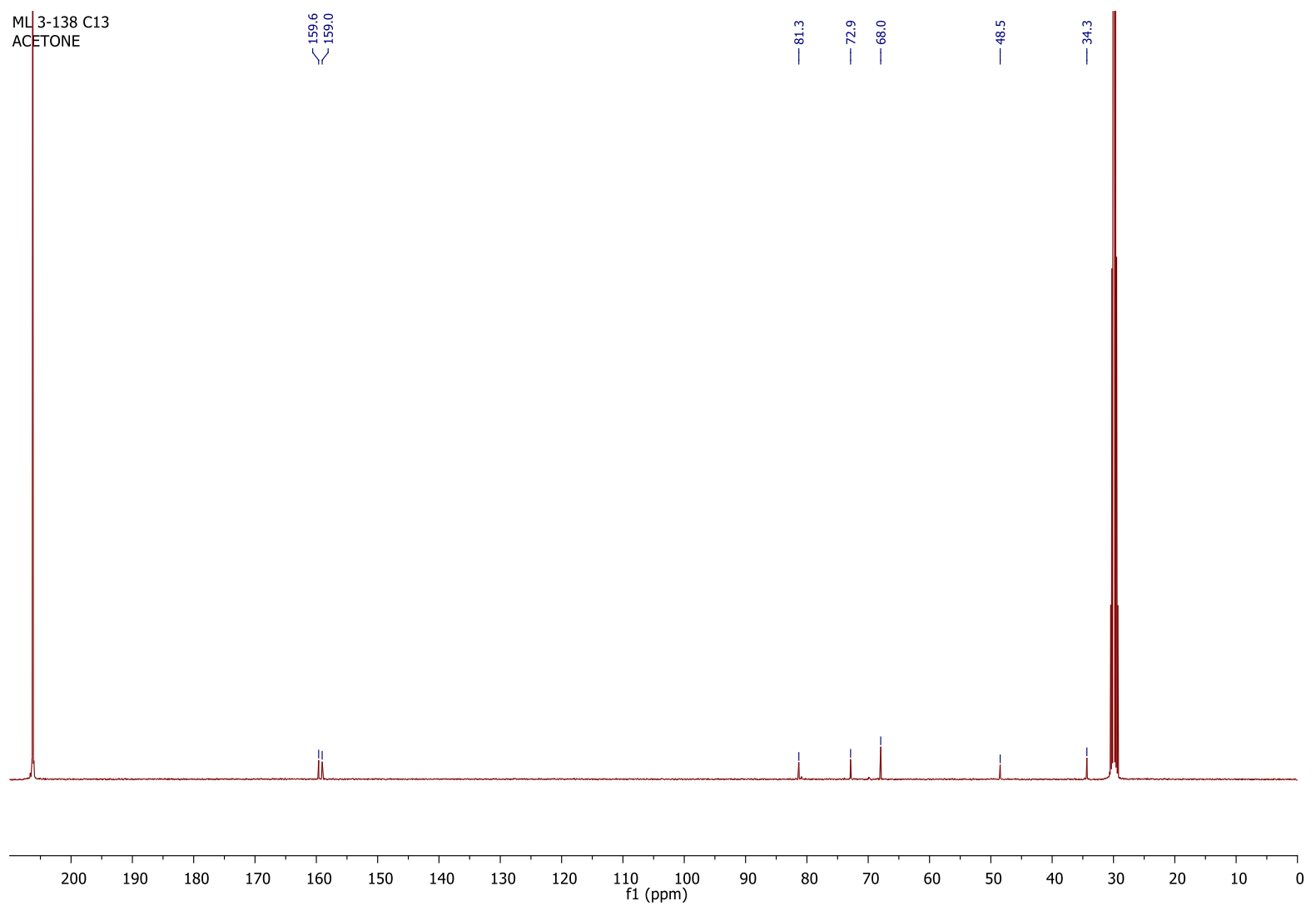

S23 
Octakis (1-((4-ethoxy-4-oxobutyl)-1H-1,2,3-triazol-4-yl)methyl) bambus[4]uril $\underline{8 a}$ ( ${ }^{1} \mathrm{H}$ NMR $400 \mathrm{MHz}, \mathrm{CDCl}_{3}$ )

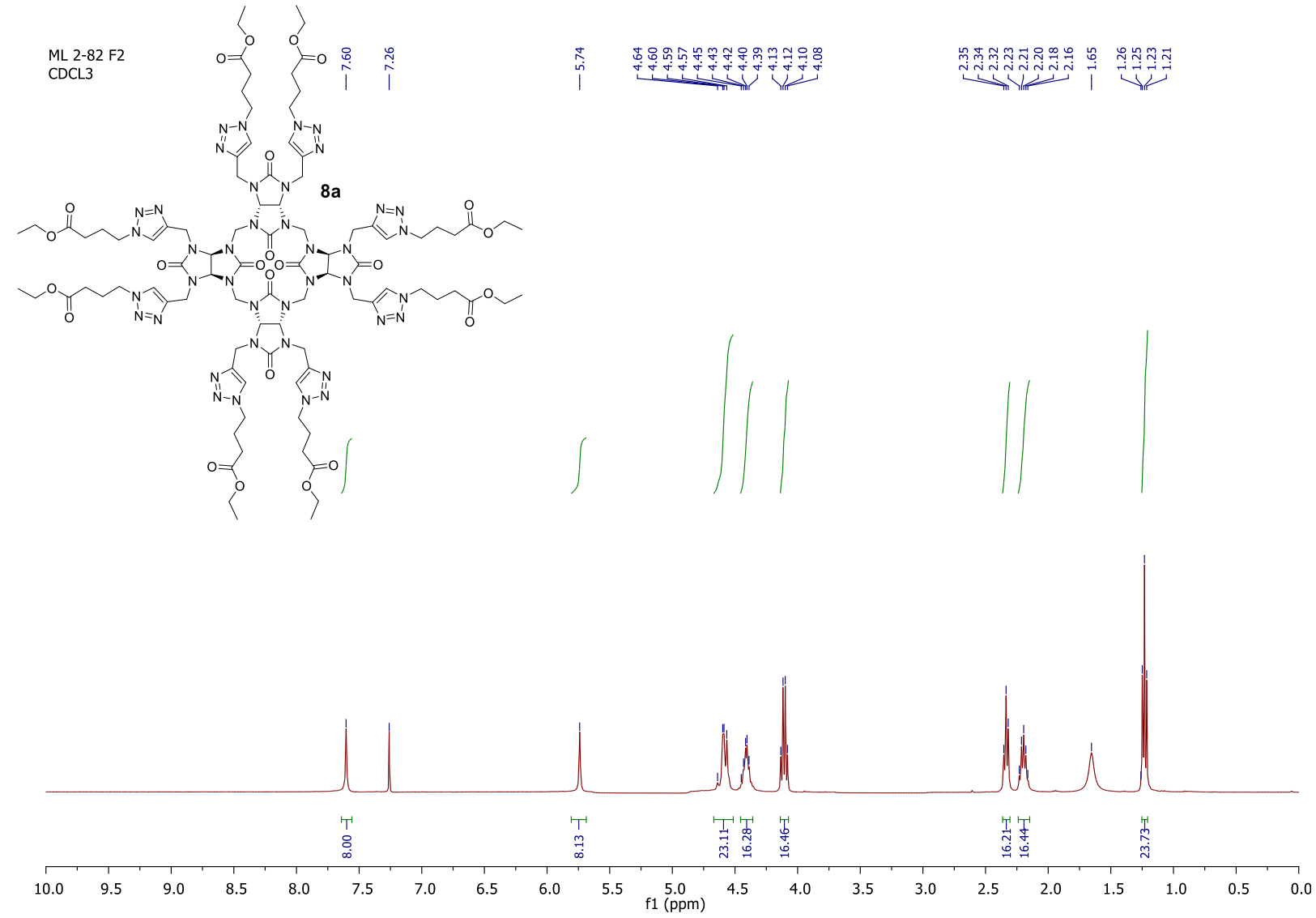

Octakis (1-((4-ethoxy-4-oxobutyl)-1H-1,2,3-triazol-4-yl)methyl) bambus[4]uril $\underline{8 a}$ $\left({ }^{13} \mathrm{C} \mathrm{NMR} 100 \mathrm{MHz}, \mathrm{CDCl}_{3}\right)$

$\mathrm{ML} 2-82 \mathrm{C} 13$

$\mathrm{CDCL3}$

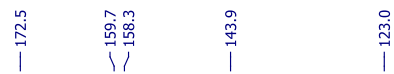

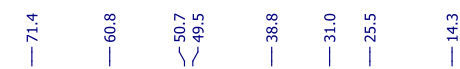

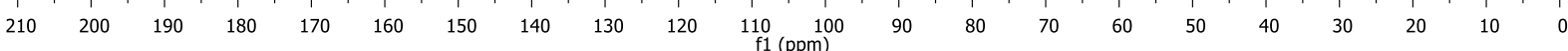


Octakis (1-(1- $\beta$-tetraacetylated glucose)-1H-1,2,3-triazol-4-yl)methyl) bambus[4]uril $\underline{8 b}$ $\left({ }^{1} \mathrm{H}\right.$ NMR $\left.400 \mathrm{MHz}, \mathrm{CDCl}_{3}\right)$

ML 2-193 F3

i

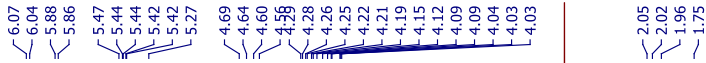
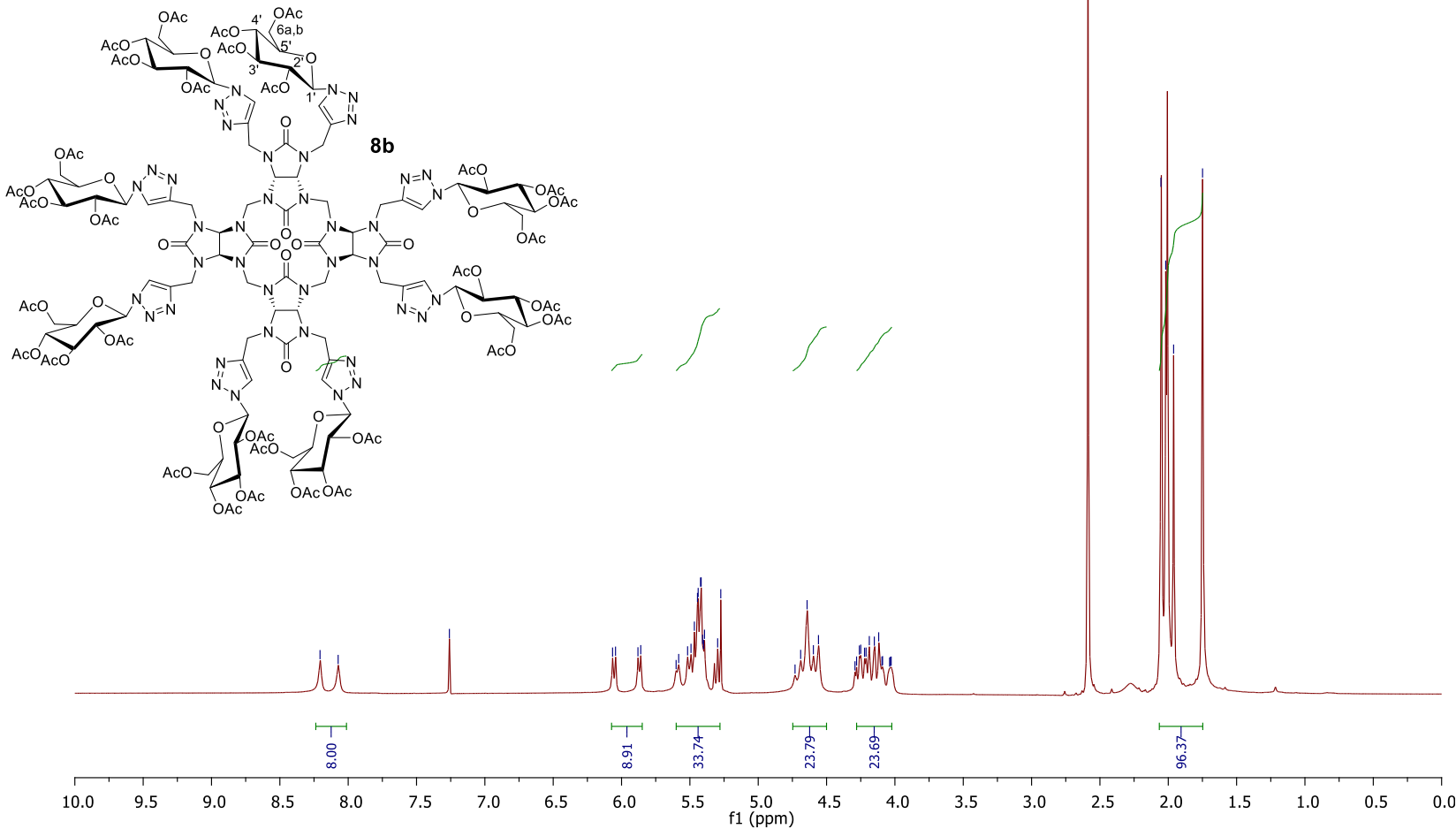

Octakis (1-(1- $\beta$-tetraacetylated glucose)-1H-1,2,3-triazol-4-yl)methyl) bambus[4]uril $\underline{8 b}$ $\left({ }^{13} \mathrm{C}\right.$ NMR $\left.100 \mathrm{MHz}, \mathrm{CDCl}_{3}\right)$

ML 2-193 F3 C13

$\mathrm{CDCL3}$

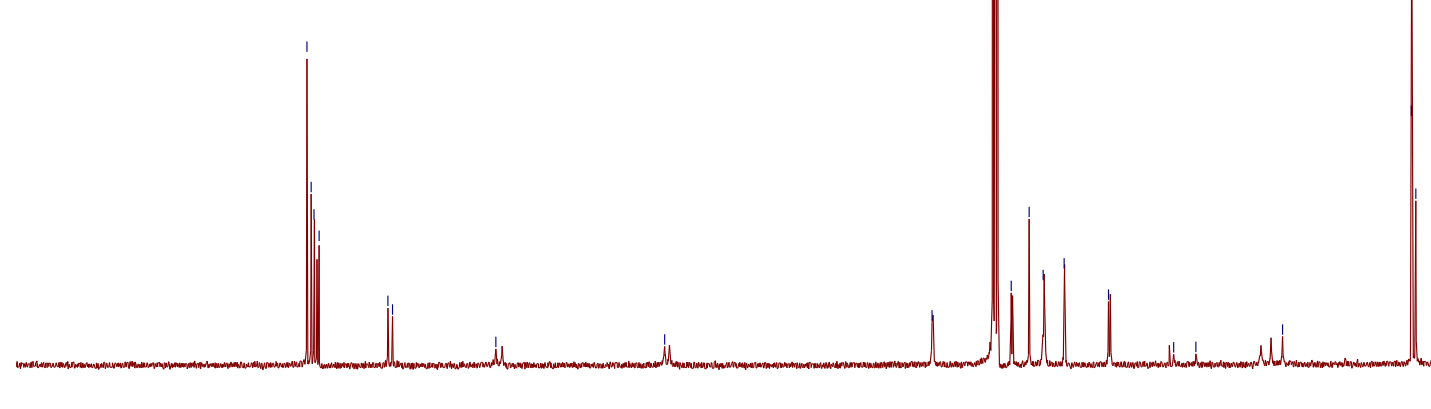


HSQC of octakis (1-(1- $\beta$-tetraacetylated glucose)-1H-1,2,3-triazol-4-yl)methyl) bambus[4]uril $\underline{8 b}$

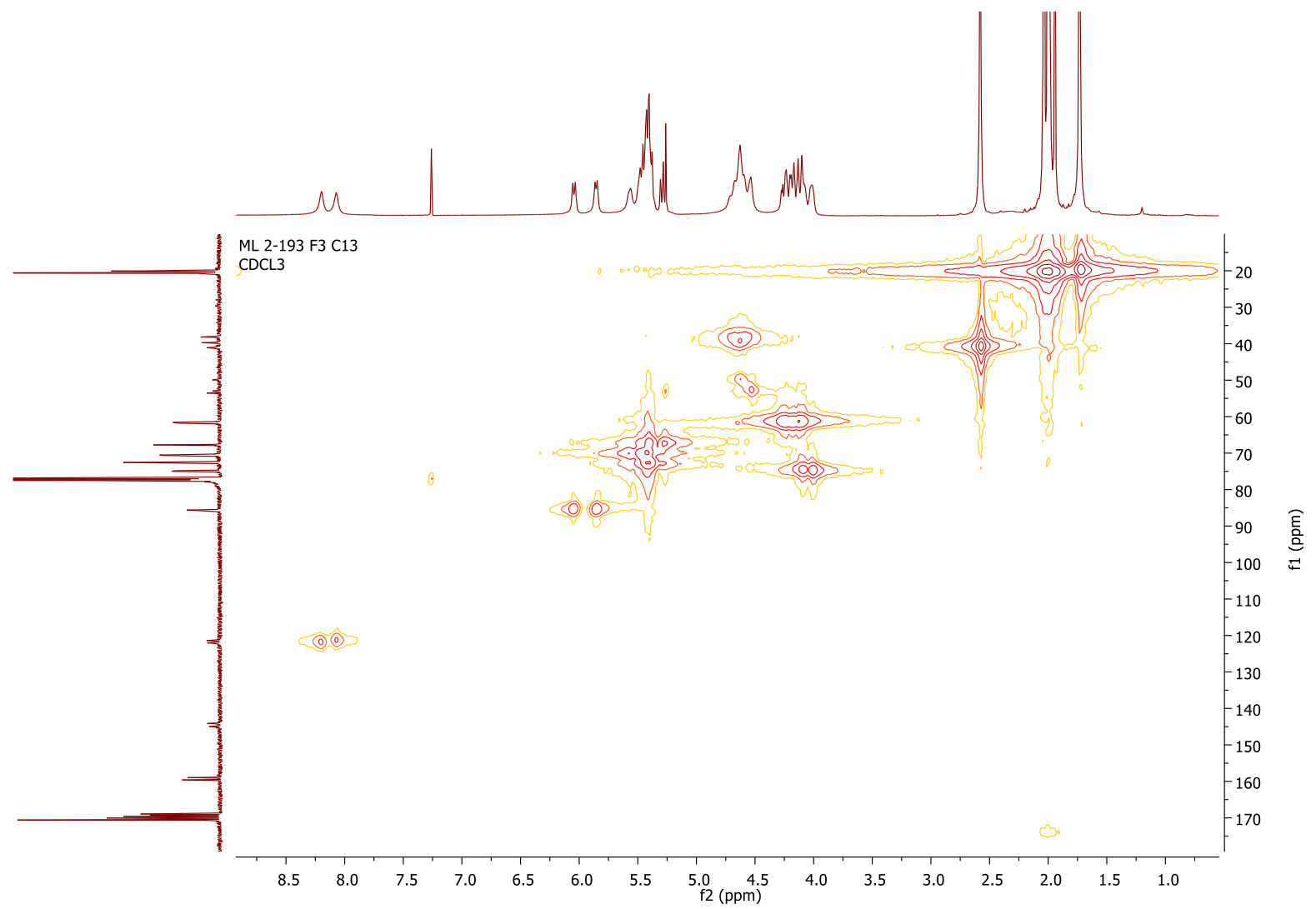


Octakis (1-(2-ethyl-2,3,4,6-tetra-0-acetyl- $\beta$-D-glucopyranoside)-1H-1,2,3-triazol4-yl)methyl) bambus[4] uril 8c ( ${ }^{1} \mathrm{H}$ NMR $\left.400 \mathrm{MHz}, \mathrm{CDCl}_{3}\right)$

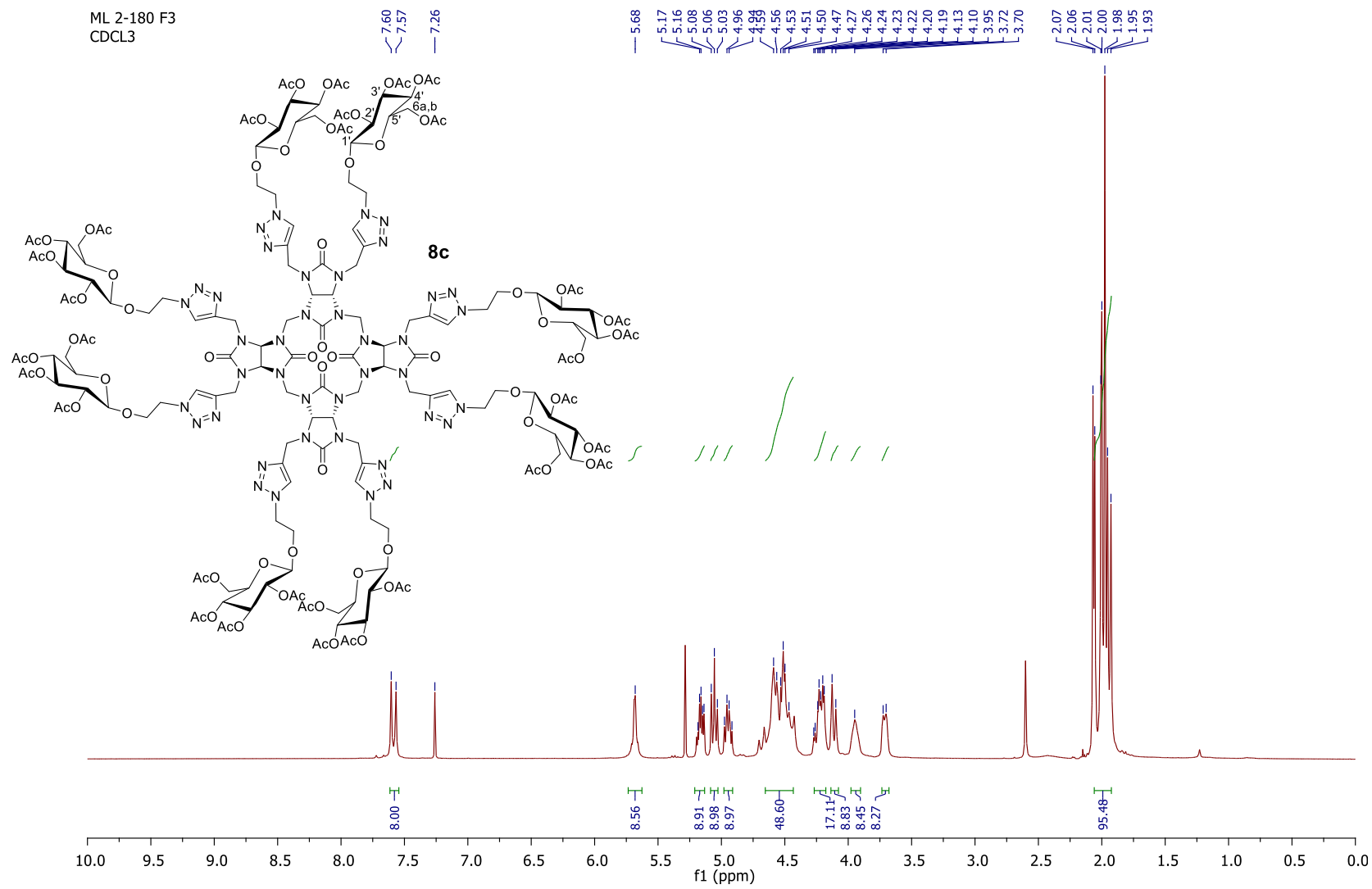

Octakis (1-(2-ethyl-2,3,4,6-tetra-0-acetyl- $\beta$-D-glucopyranoside)-1H-1,2,3-triazol4-yl)methyl) bambus[4]uril $8 \mathrm{c}\left({ }^{13} \mathrm{C} N \mathrm{NR} 100 \mathrm{MHz}, \mathrm{CDCl}_{3}\right)$

ML 2-180 F3 C13 CDCL3 
HSQC of octakis (1-(2-ethyl-2,3,4,6-tetra-O-acetyl- $\beta$-D-glucopyranoside)-1H-1,2,3-triazol4-yl)methyl) bambus[4]uril $\underline{8 \mathrm{c}}$

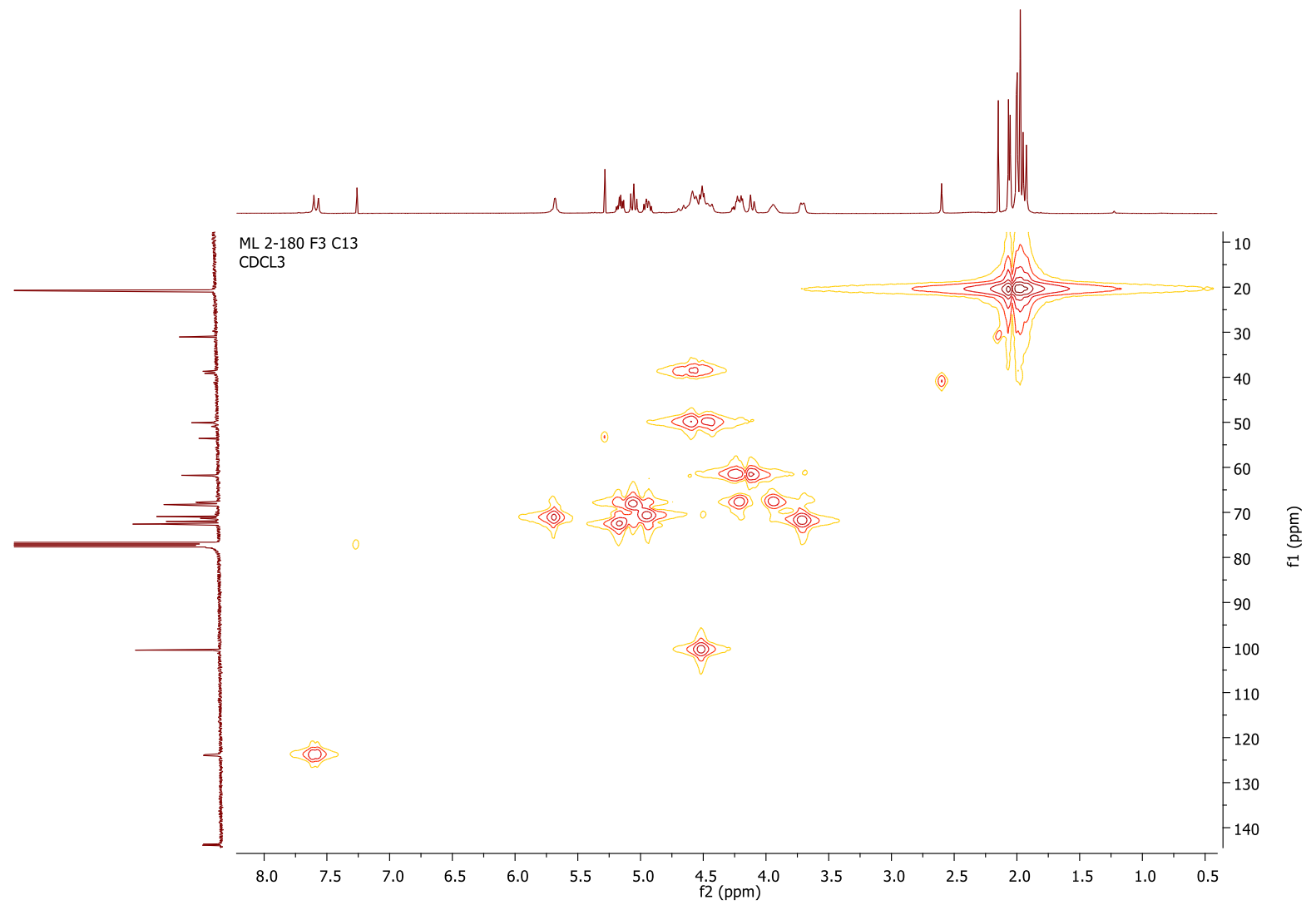


Sodium bromide @Dodecakis 1-((4-ethoxy-4-oxobutyl)-1H-1,2,3-triazol-4-yl)methyl) bambus[6]uril 9a $\left({ }^{1} \mathrm{H}\right.$ NMR $\left.400 \mathrm{MHz}, \mathrm{CDCl}_{3}\right)$

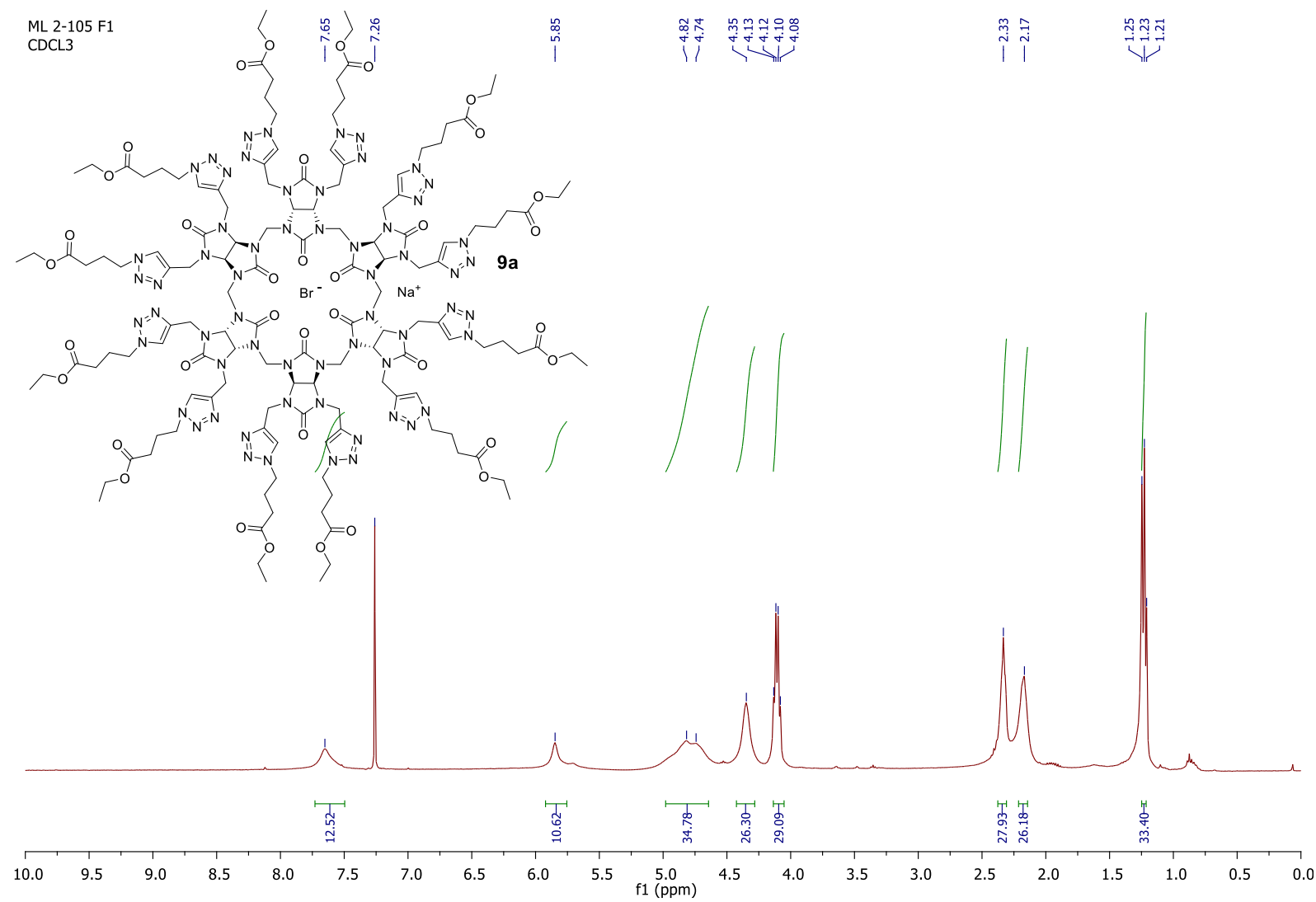

Sodium bromide @Dodecakis 1-((4-ethoxy-4-oxobutyl)-1H-1,2,3-triazol-4-yl)methyl) bambus[6]uril 9a $\left({ }^{13} \mathrm{C} \mathrm{NMR} 100 \mathrm{MHz}, \mathrm{CDCl}_{3}\right)$

ML 2-105 F1 C13

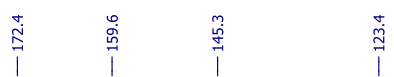

lidion 
Sodium bromide @Dodecakis 1-((4-ethoxy-4-oxobutyl)-1H-1,2,3-triazol-4-yl)methyl) bambus[6]uril 10a $\left({ }^{1} \mathrm{H}\right.$ NMR $\left.400 \mathrm{MHz}, \mathrm{CDCl}_{3}\right)$

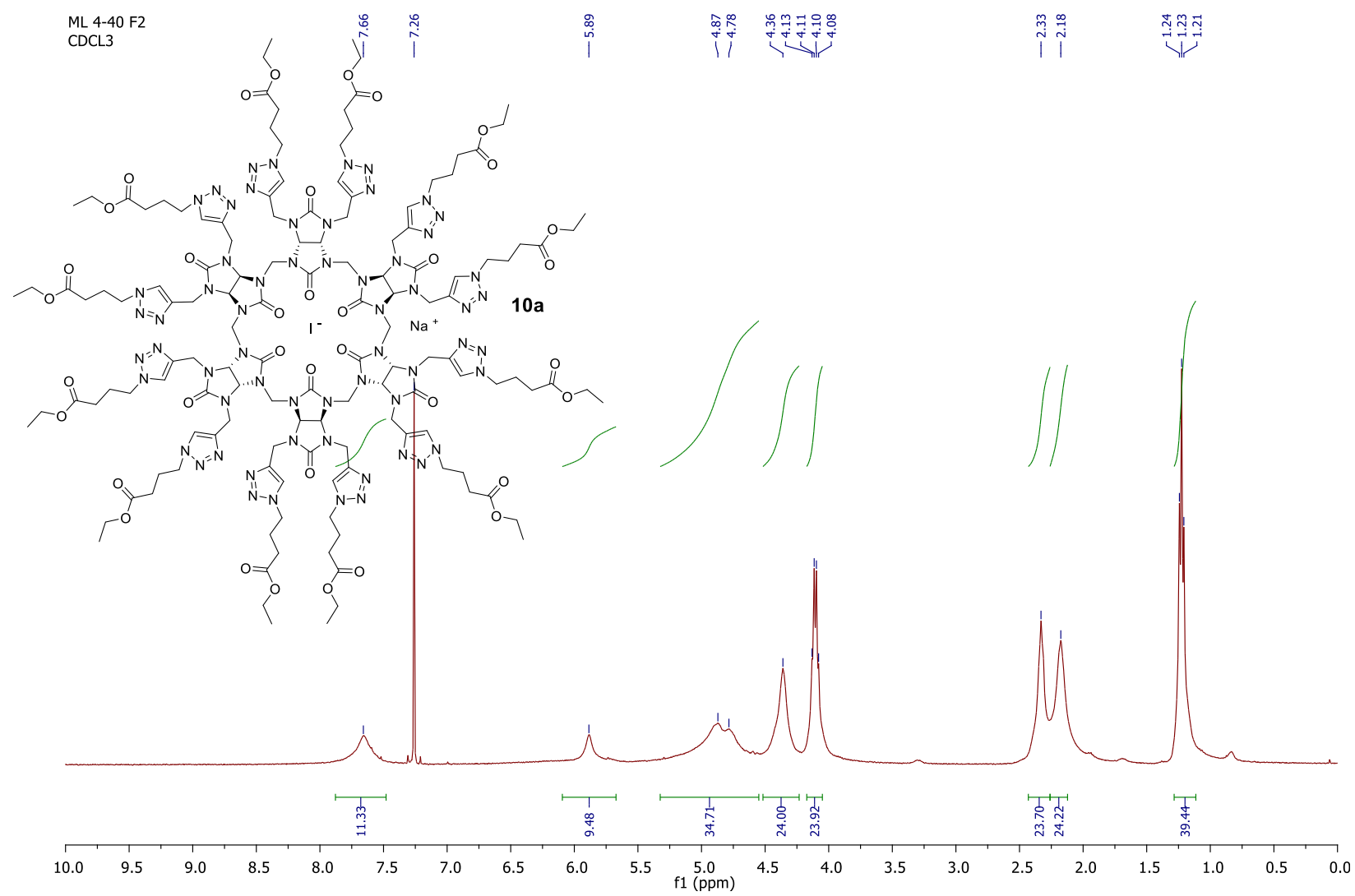

Sodium bromide @Dodecakis 1-((4-ethoxy-4-oxobutyl)-1H-1,2,3-triazol-4-yl)methyl) bambus[6]uril 10a $\left({ }^{13} \mathrm{C} \mathrm{NMR} 100 \mathrm{MHz}, \mathrm{CDCl}_{3}\right)$

$M L-4-40-F 2$ 
Sodium bromide @Dodecakis (1-(2-ethyl-2,3,4,6-tetra-O-acetyl- $\beta$-D-glucopyranoside)-1H-1,2,3triazol-4-yl)methyl) bambus[6]uril $9 \mathrm{c}\left({ }^{1} \mathrm{H}\right.$ NMR $\left.400 \mathrm{MHz}, \mathrm{CDCl}_{3}\right)$

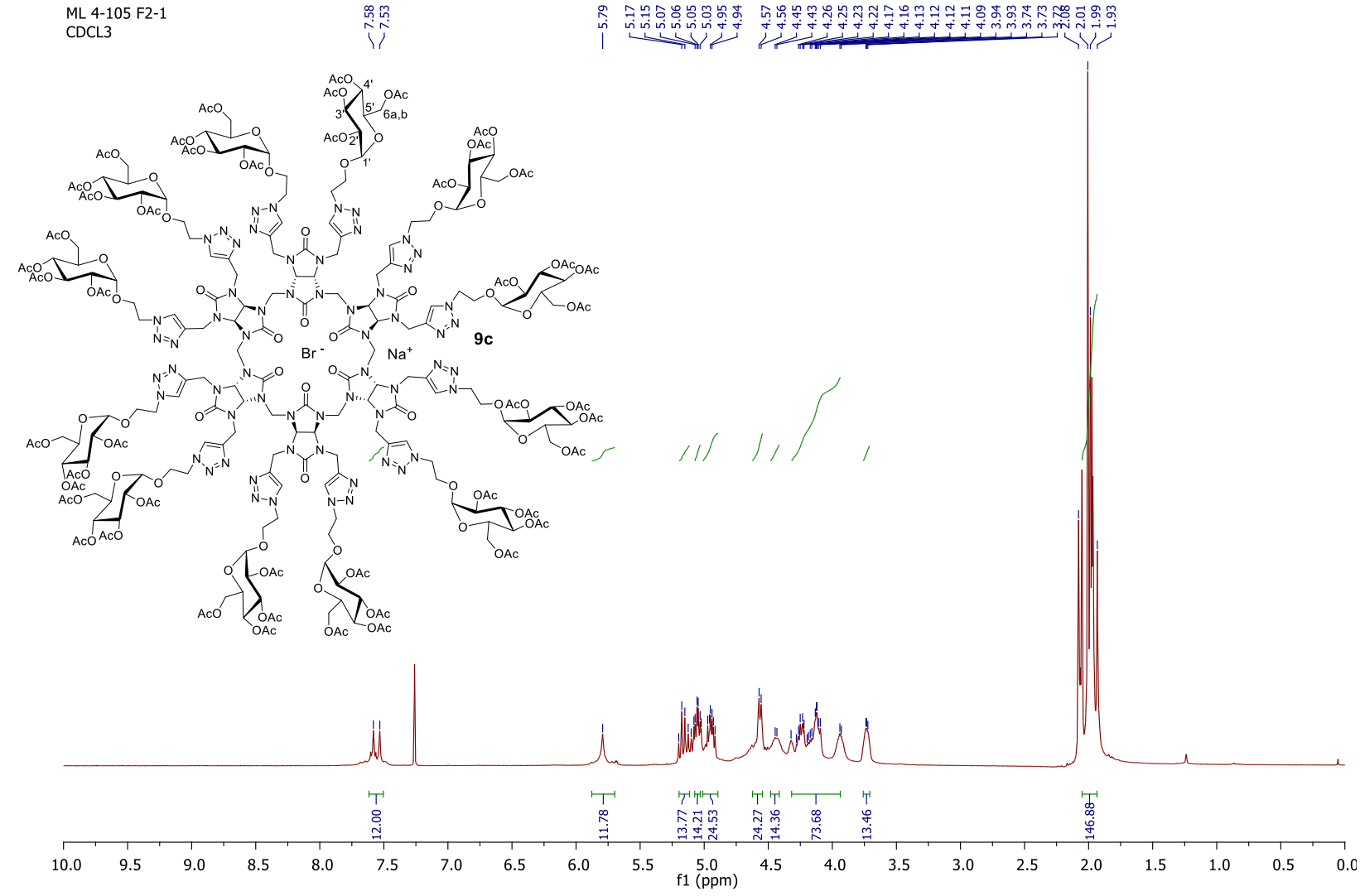

Sodium bromide @Dodecakis (1-(2-ethyl-2,3,4,6-tetra-O-acetyl- $\beta$-D-glucopyranoside)-1H-1,2,3triazol-4-yl)methyl) bambus[6]uril $\underline{9 \mathrm{c}}\left({ }^{13} \mathrm{C} \mathrm{NMR} 100 \mathrm{MHz}, \mathrm{CDCl}_{3}\right)$

ML 4-105 F3 C13

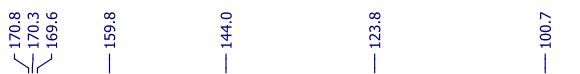

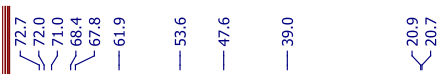

$\begin{array}{llllllllllllllllllll}200 & 190 & 180 & 170 & 160 & 150 & 140 & 130 & 120 & \underset{f}{110}(\mathrm{ppm}) & 90 & 80 & 70 & 60 & 50 & 40 & 30 & 20 & 10 & 0\end{array}$ 
HSQC of of sodium bromide @Dodecakis (1-(2-ethyl-2,3,4,6-tetra-O-acetyl- $\beta$-D-glucopyranoside)1H-1,2,3-triazol-4-yl)methyl) bambus[6]uril 9c

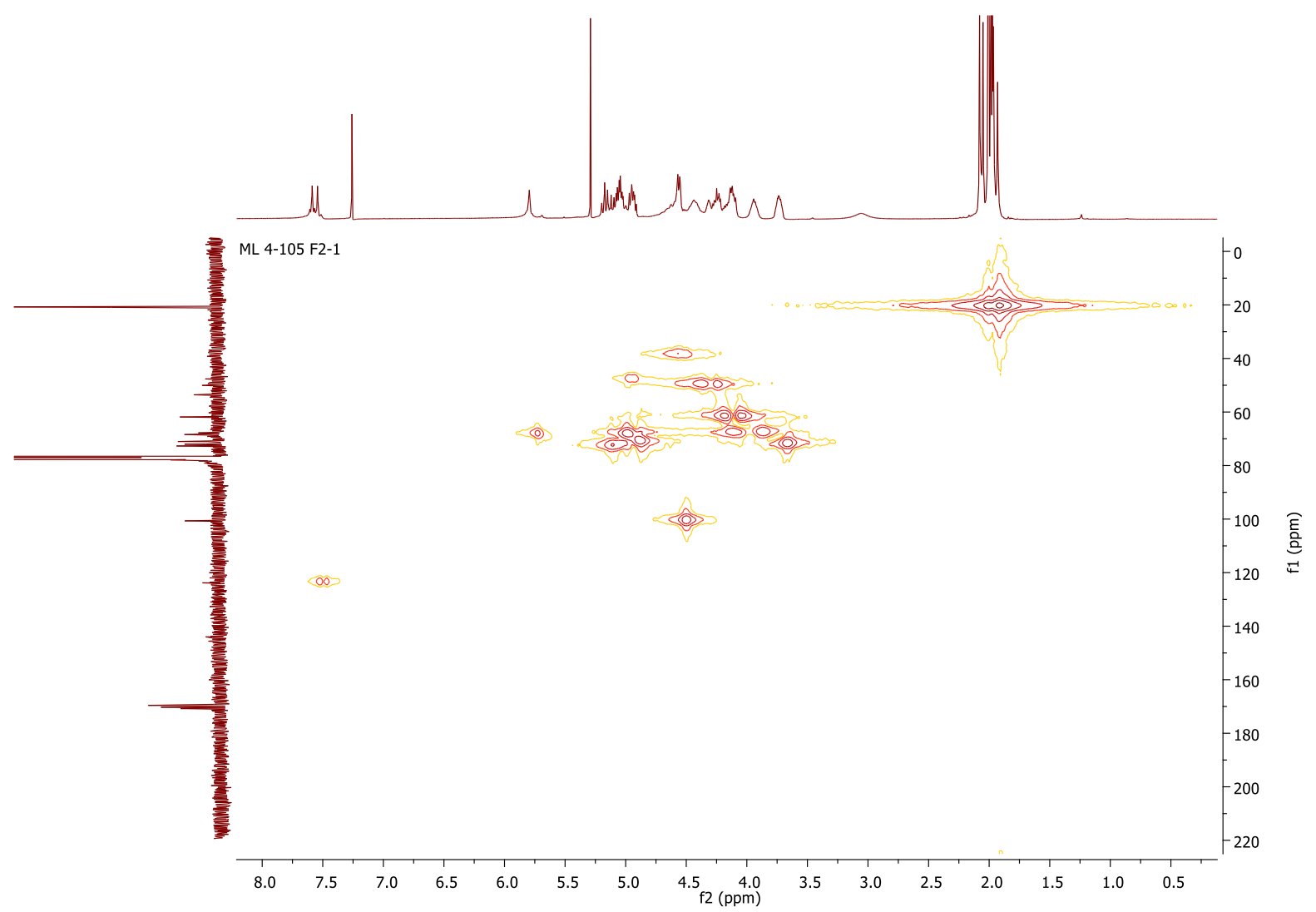


Dodecakis 1-((4-ethoxy-4-oxobutyl)-1H-1,2,3-triazol-4-yl)methyl) bambus[6]uril 11a ( ${ }^{1} \mathrm{H}$ NMR $400 \mathrm{MHz}, \mathrm{CDCl}_{3}$ )

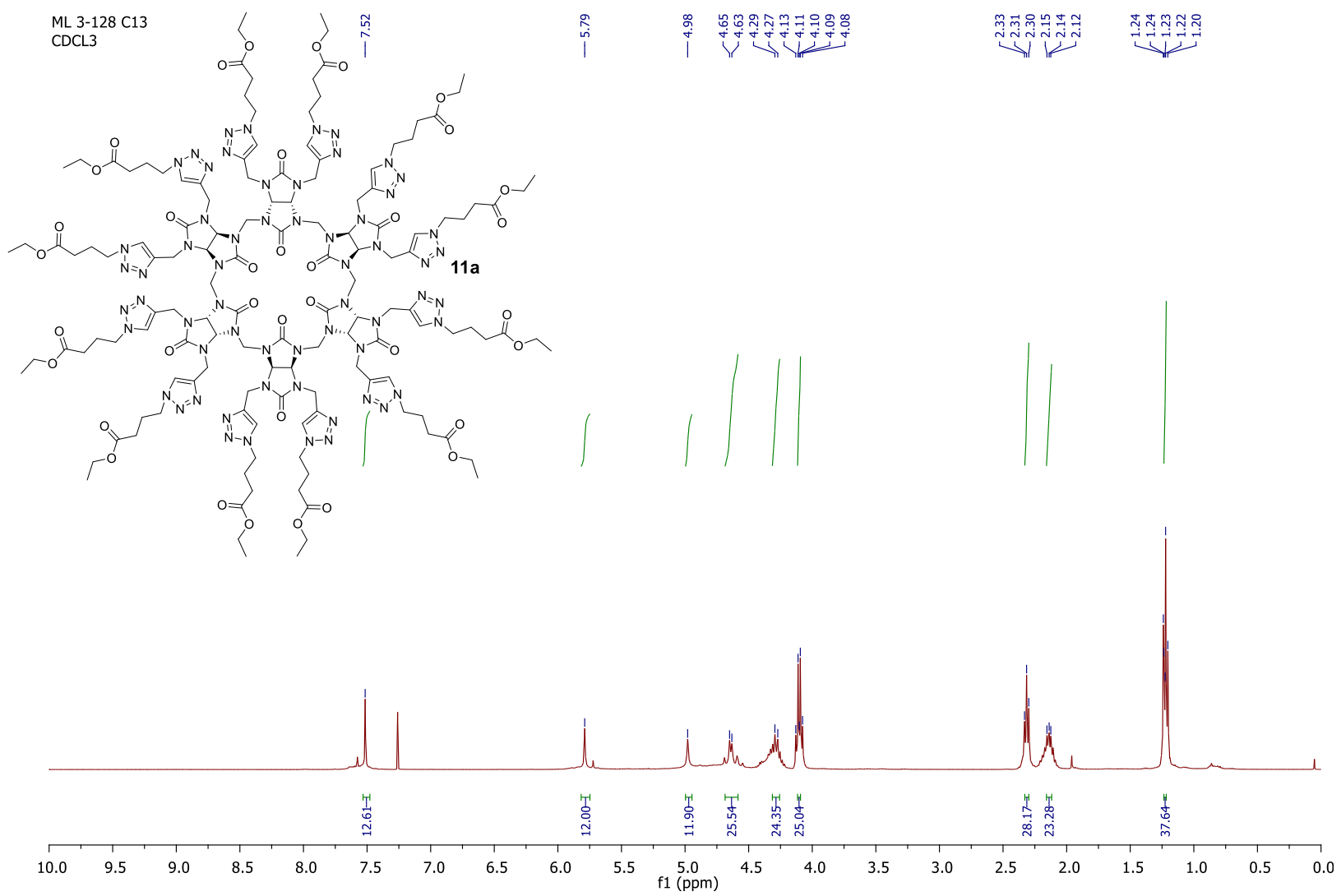

Dodecakis 1-((4-ethoxy-4-oxobutyl)-1H-1,2,3-triazol-4-yl)methyl) bambus[6]uril 11a $\left({ }^{13} \mathrm{C}\right.$ NMR $100 \mathrm{MHz}, \mathrm{CDCl}_{3}$ )

ML 3-128 C13

CDCL3

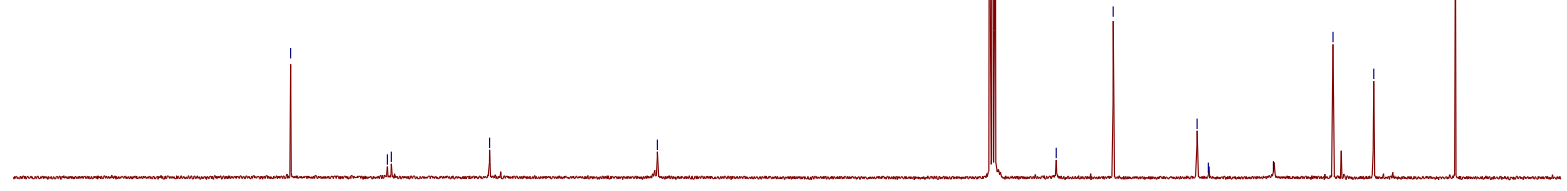

$\begin{array}{llllllllllllllllllllllll}210 & 200 & 190 & 180 & 170 & 160 & 150 & 140 & 130 & 120 & 110 & 100 & 90 & 80 & 70 & 60 & 50 & 40 & 30 & 20 & 10 & 0\end{array}$ 
HSQC of dodecakis 1-((4-ethoxy-4-oxobutyl)-1H-1,2,3-triazol-4-yl)methyl) bambus[6]uril 11a

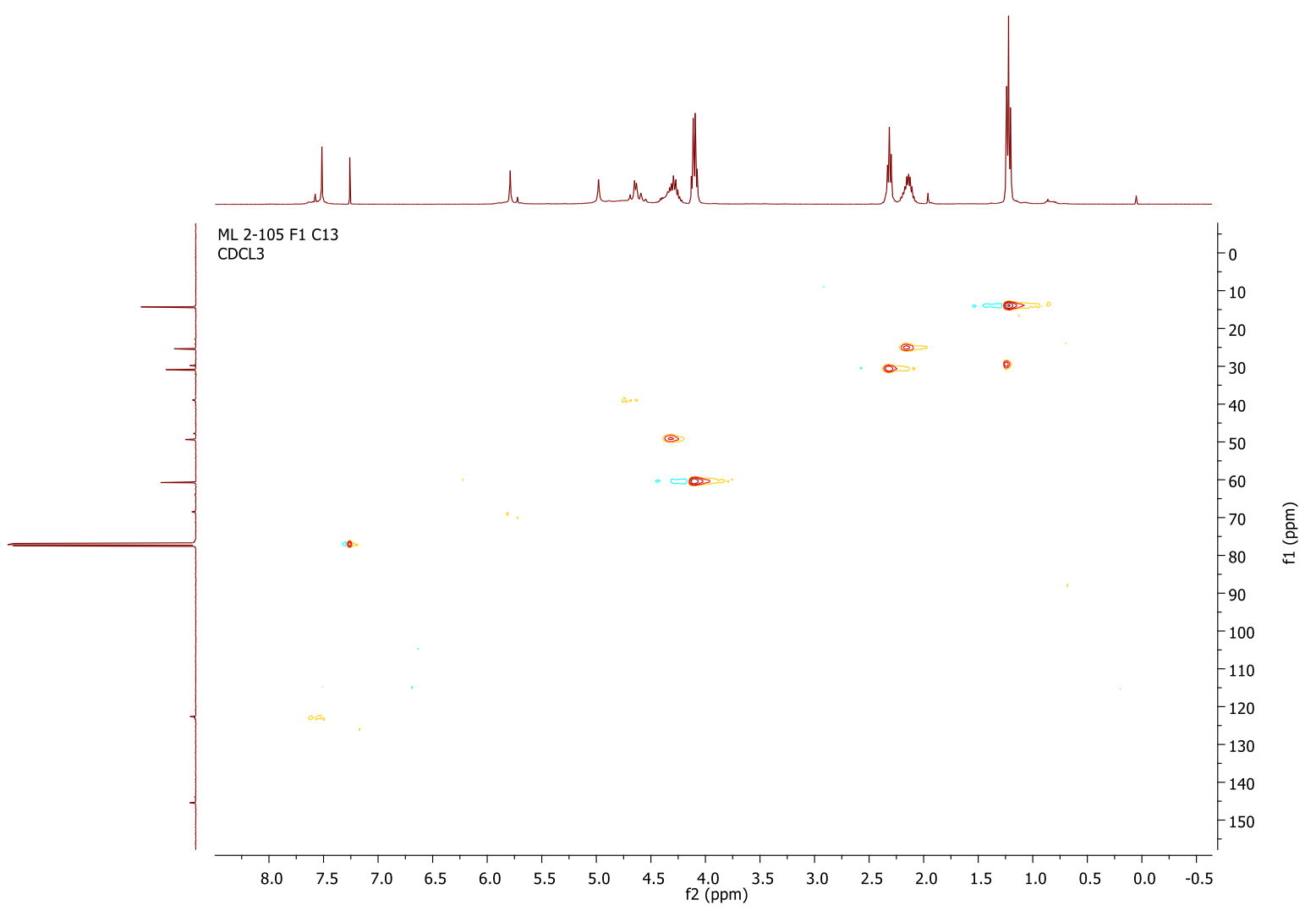


Dodecakis (1-(1- $\beta$-tetraacetylated glucose)-1H-1,2,3-triazol-4-yl)methyl)bambus[6]uril $\underline{11 \mathrm{~b}}\left({ }^{1} \mathrm{H}\right.$ NMR $\left.400 \mathrm{MHz}, \mathrm{CDCl}_{3}\right)$

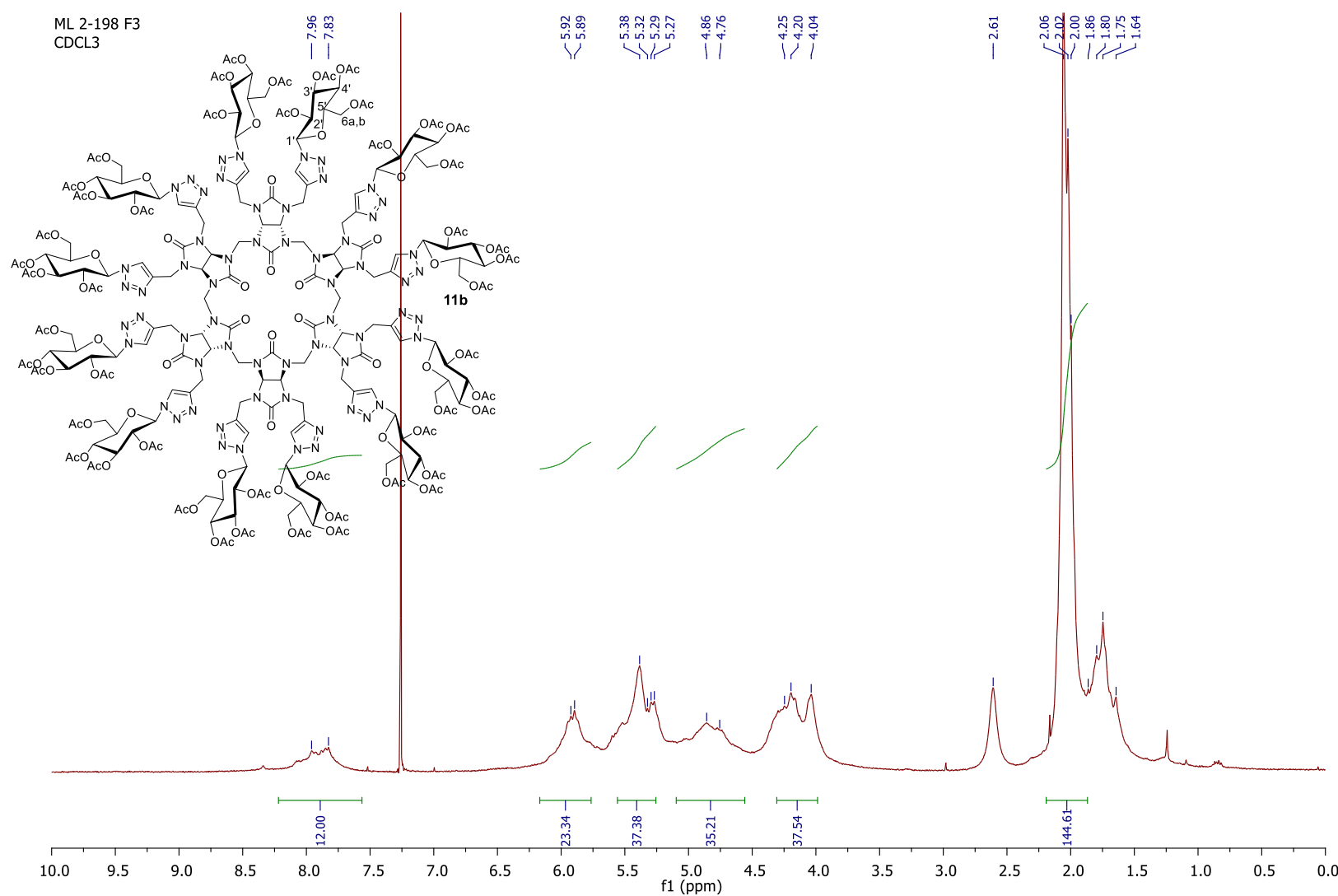

Dodecakis (1-(1- $\beta$-tetraacetylated glucose)-1H-1,2,3-triazol-4-yl)methyl)bambus[6]uril $\underline{11 \mathrm{~b}}\left({ }^{13} \mathrm{C} \mathrm{NMR} 100 \mathrm{MHz}, \mathrm{CDCl}_{3}\right)$

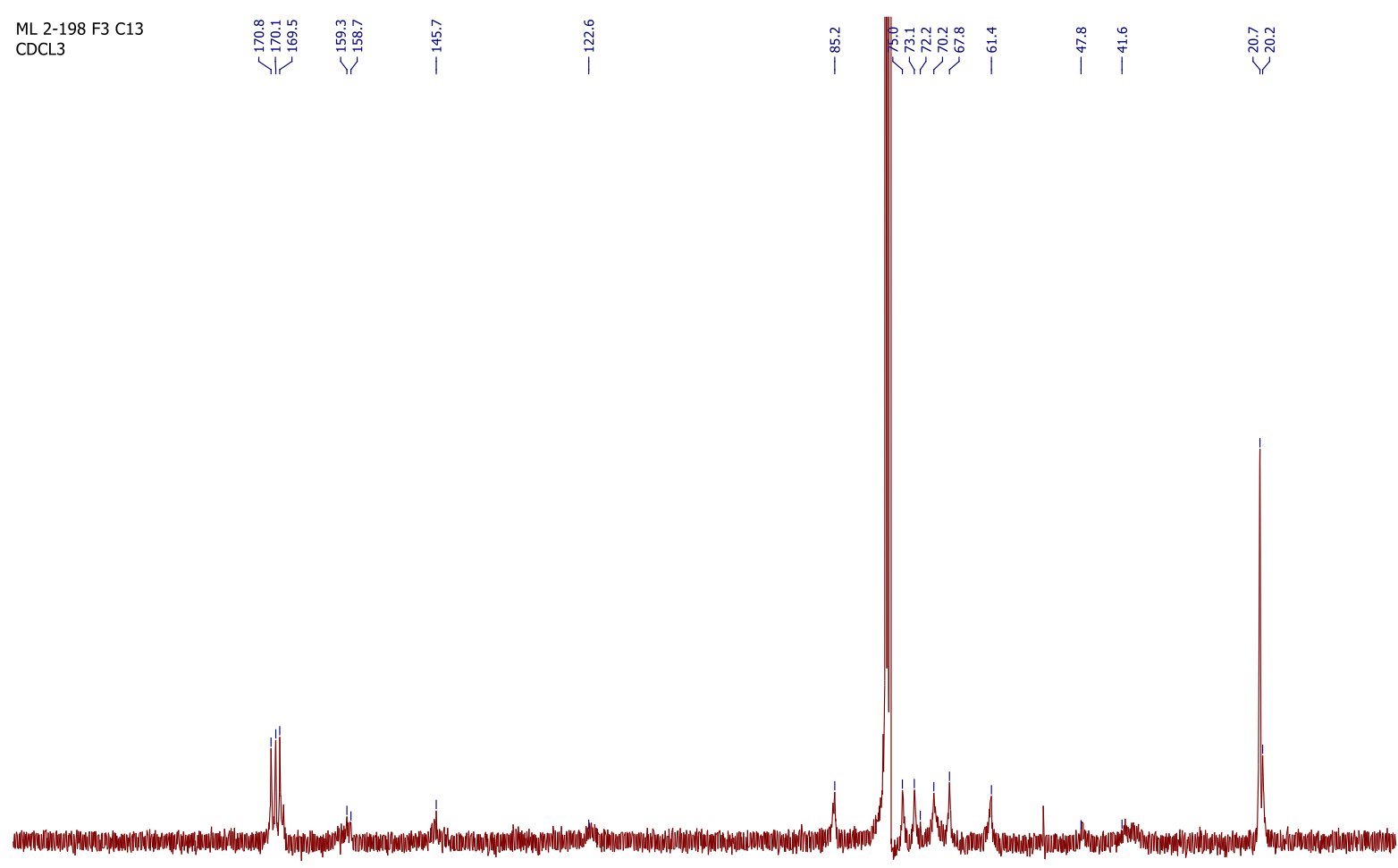

\begin{tabular}{rlllllllllllllllllllll}
\hline 210 & 200 & 190 & 180 & 170 & 160 & 150 & 140 & 130 & 120 & $\underset{\mathrm{f} 1}{110}(\mathrm{ppm})$ & 100 & 90 & 80 & 70 & 60 & 50 & 40 & 30 & 20 & 10 & 0
\end{tabular} 
HSQC of dodecakis (1-(1- $\beta$-tetraacetylated glucose)-1H-1,2,3-triazol-4yl)methyl)bambus[6]uril 11b

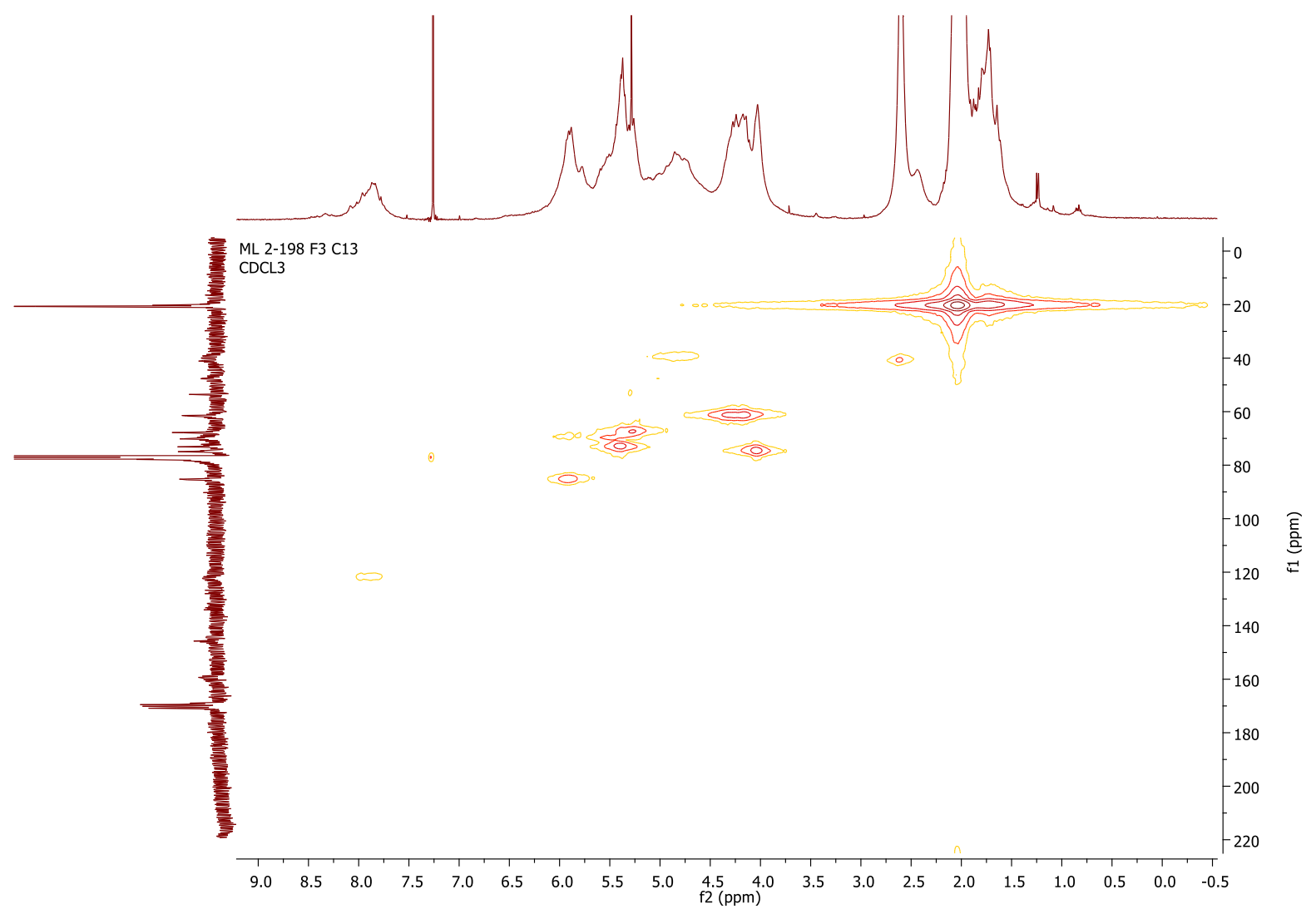


Dodecakis (1-(2-ethyl-2,3,4,6-tetra-O-acetyl- $\beta$-D-glucopyranoside)-1H-1,2,3-triazol4-yl)methyl) bambus[6]uril 11c $\left({ }^{1} \mathrm{H}\right.$ NMR $\left.400 \mathrm{MHz}, \mathrm{CDCl}_{3}\right)$

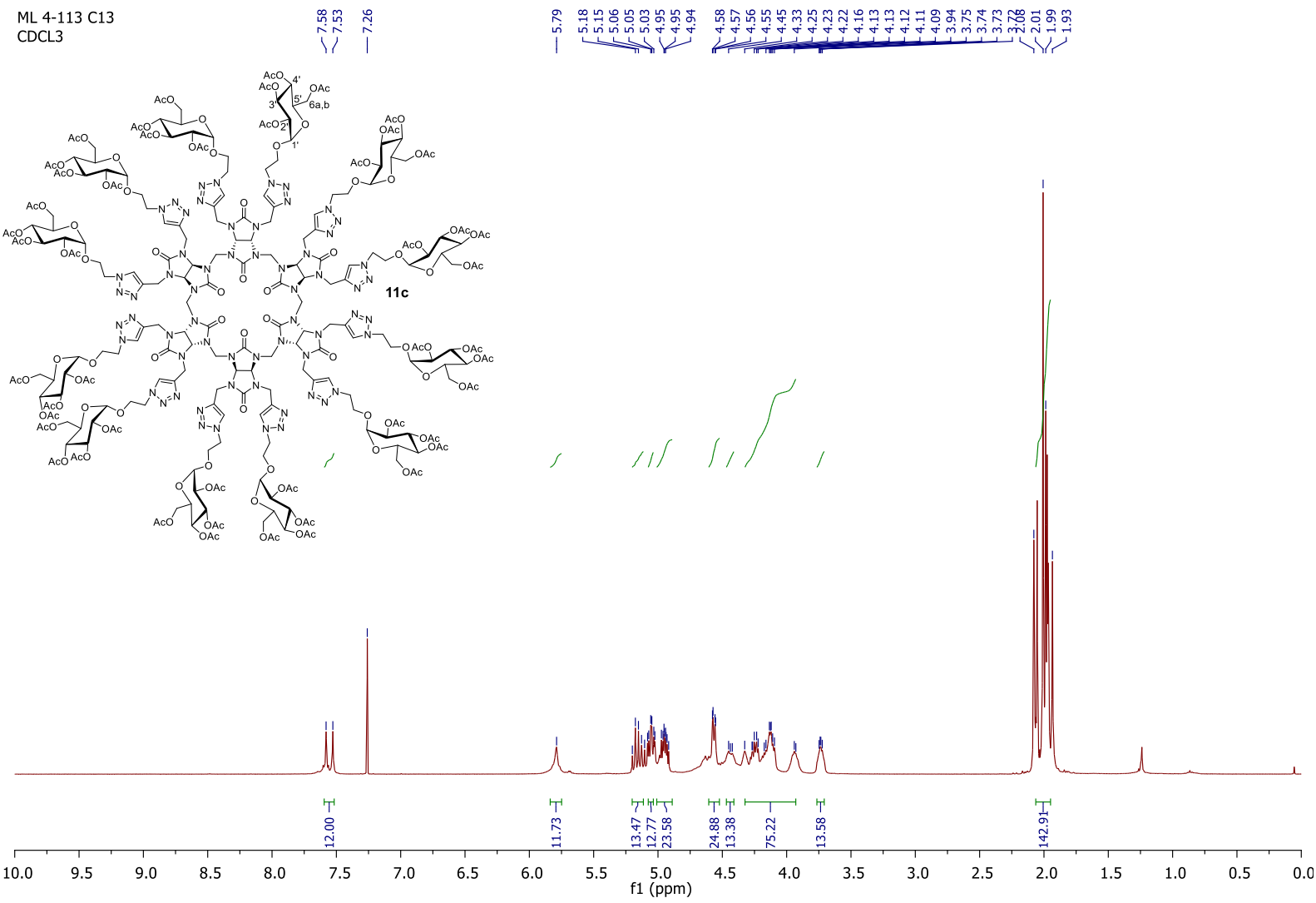

Dodecakis (1-(2-ethyl-2,3,4,6-tetra-0-acetyl- $\beta$-D-glucopyranoside)-1H-1,2,3-triazol4-yl)methyl) bambus[6]uril $\underline{11 \mathrm{C}}\left({ }^{13} \mathrm{C}\right.$ NMR $\left.100 \mathrm{MHz}, \mathrm{CDCl}_{3}\right)$

ML 4-113 C13

CDCL3

|

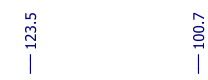

||cind

$\begin{array}{rllllllllll}210 & 200 & 190 & 180 & 170 & 160 & 150 & 140 & 130 & 120 & 110 \\ \mathrm{f} 1(\mathrm{ppm}) & 100\end{array}$ 
Dodecakis (1-(4 butanoic acid)-1H-1,2,3-triazol-4-yl)methyl) bambus[6]uril $\underline{12}\left({ }^{1} \mathrm{H}\right.$ NMR $400 \mathrm{MHz}, \mathrm{D}_{2} \mathrm{O}$ )

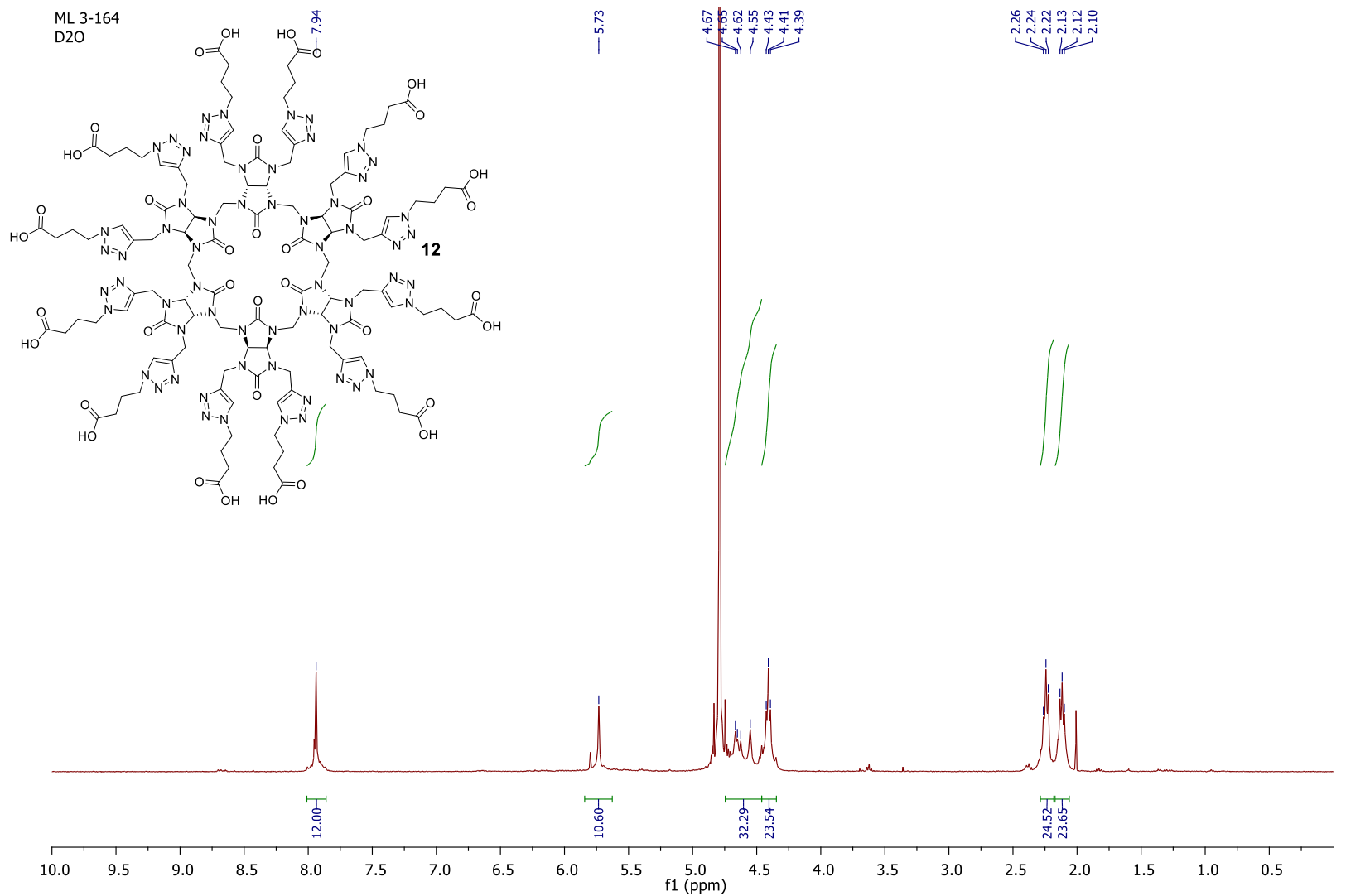

Dodecakis (1-(4 butanoic acid)-1H-1,2,3-triazol-4-yl)methyl) bambus[6]uril $\underline{12}\left({ }^{13} \mathrm{C}\right.$ NMR $100 \mathrm{MHz}, \mathrm{D}_{2} \mathrm{O}$ )

ML 3-164 C13

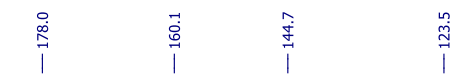

i.jul

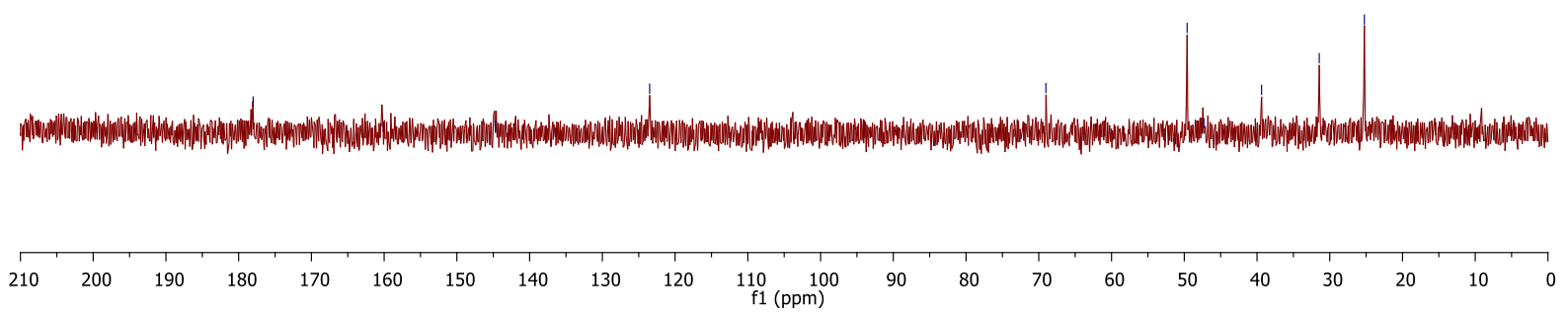


Dodecakis (1-(2-ethyl- $\beta$-D-glucopyranoside)-1H-1,2,3-triazol-4 yl)methyl) bambus[6]uril $\underline{13}\left({ }^{1} \mathrm{H}\right.$ NMR $\left.400 \mathrm{MHz}, \mathrm{D}_{2} \mathrm{O}\right)$

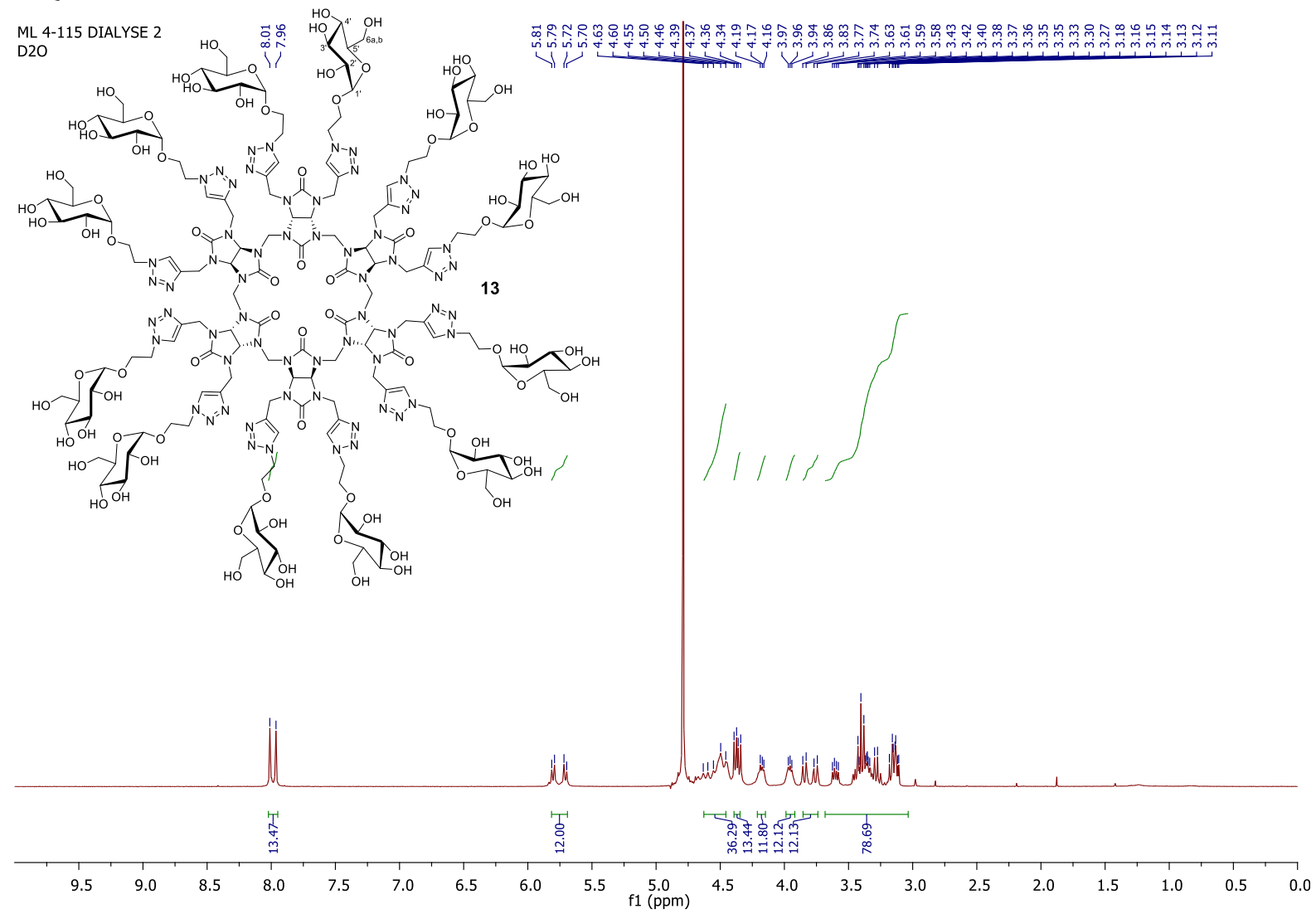

Dodecakis (1-(2-ethyl- $\beta$-D-glucopyranoside)-1H-1,2,3-triazol-4 yl)methyl) bambus[6]uril $\underline{13}\left({ }^{13} \mathrm{C} N M R 100 \mathrm{MHz}, \mathrm{D}_{2} \mathrm{O}\right)$

ML 4-115 DIALYSE 2 C13

D2O

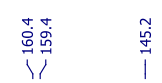

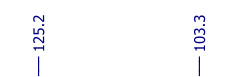

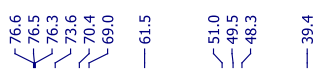

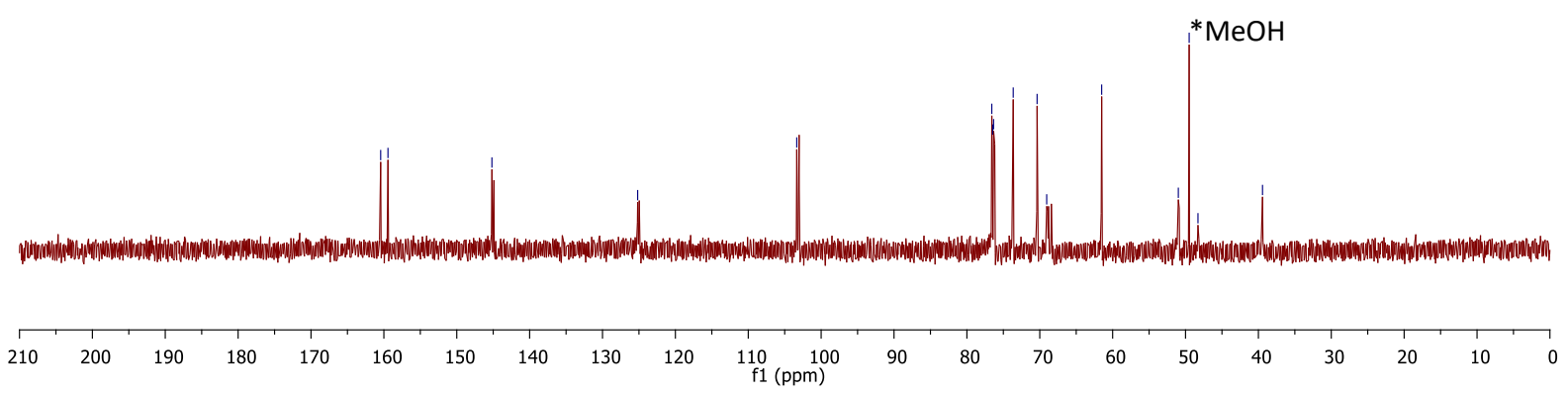


Dodecakis (1-(1- $\beta$-D-glucose)-1H-1,2,3-triazol-4-yl)methyl)bambus[6]uril 14 ( ${ }^{1} \mathrm{H}$ NMR 400 $\mathrm{MHz}, \mathrm{D}_{2} \mathrm{O}$ )

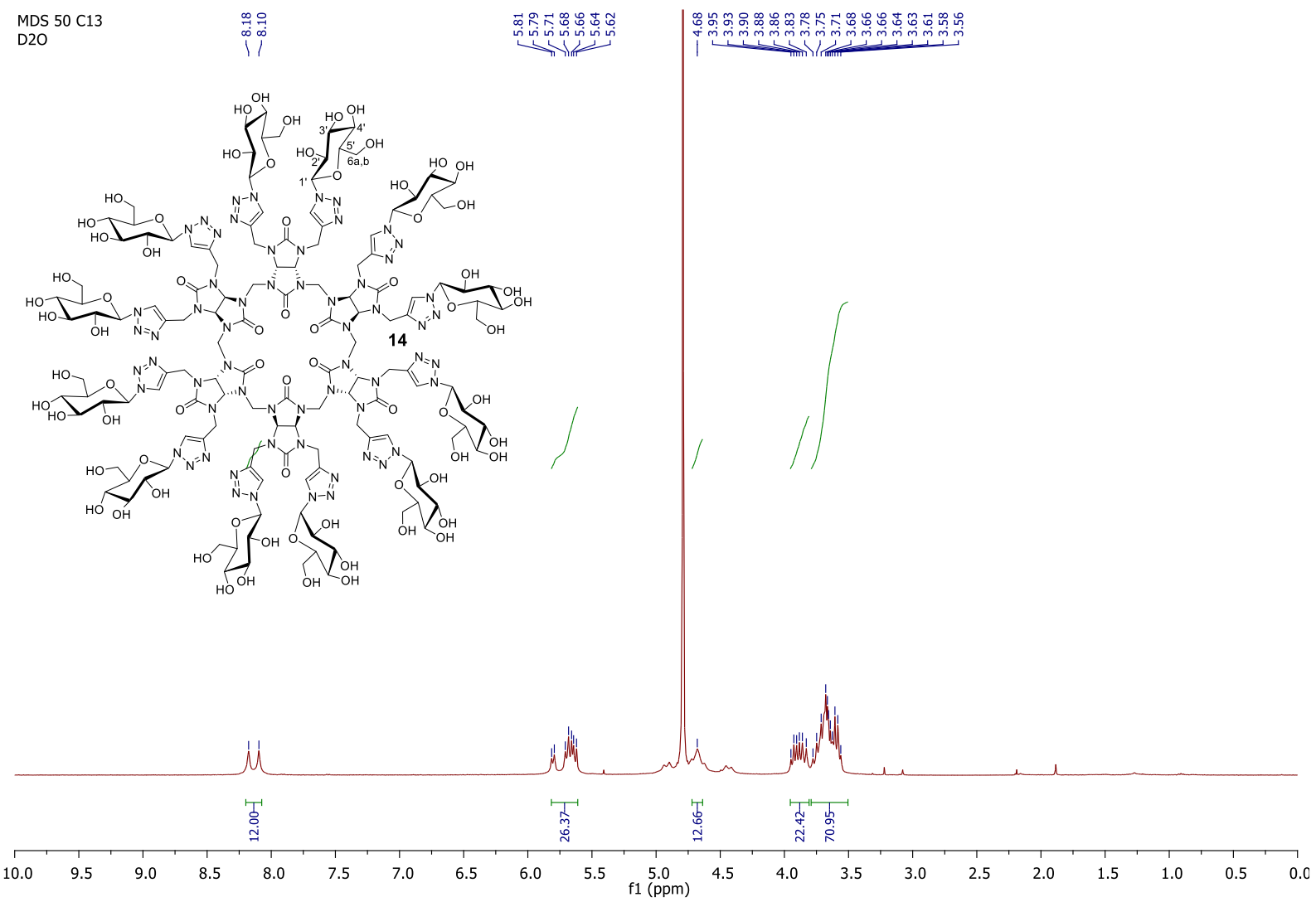

Dodecakis (1-(1- $\beta$-D-glucose)-1H-1,2,3-triazol-4-yl)methyl)bambus[6]uril $14\left({ }^{13} \mathrm{C}\right.$ NMR 100 $\mathrm{MHz}, \mathrm{D}_{2} \mathrm{O}$ )

MDS $50 \mathrm{C} 13$

D2O

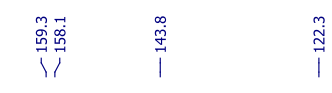

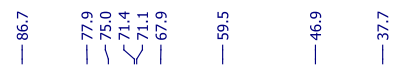

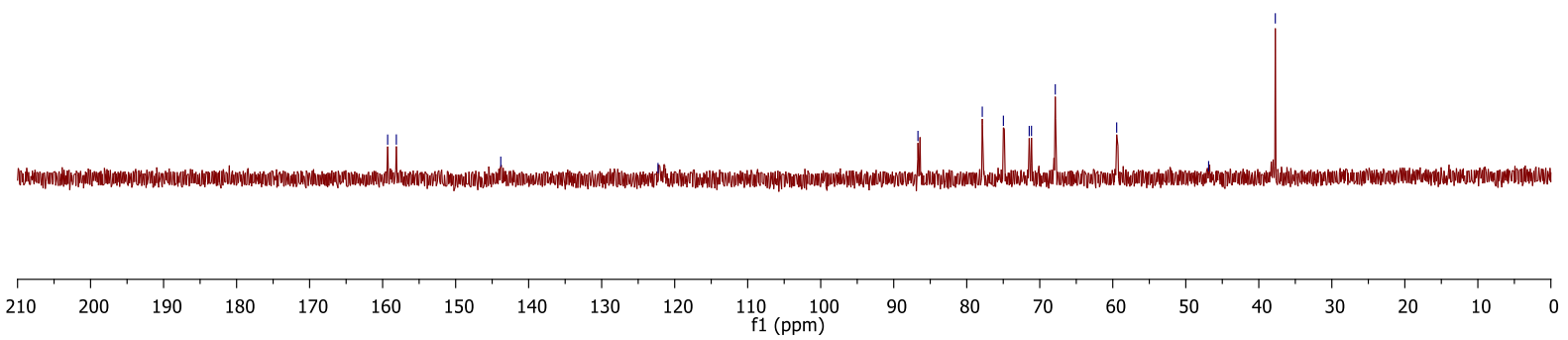




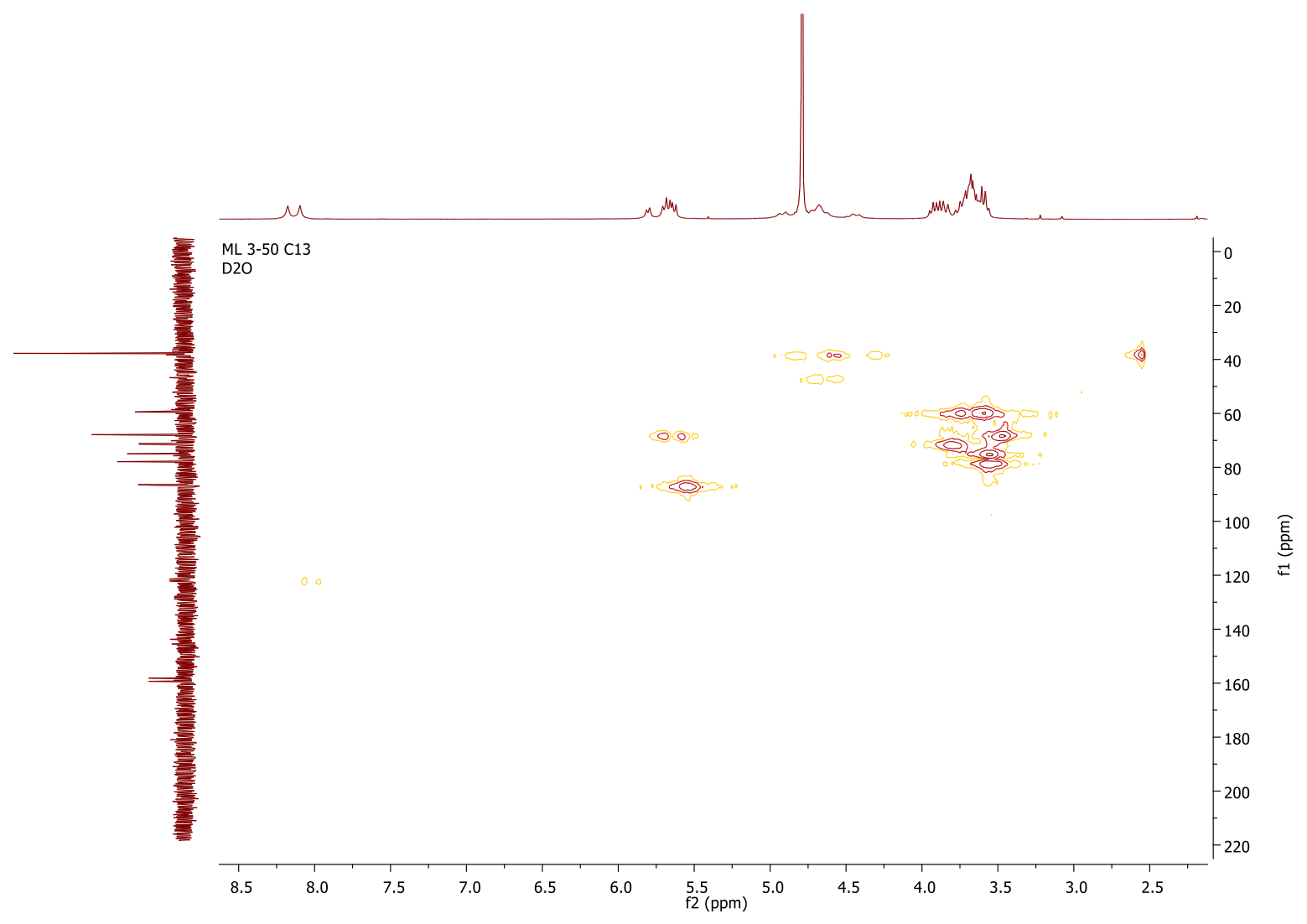

Tetrabutylammonium-bromide@Dodecakis(1-(1- $\beta$-D-glucose)-1H-1,2,3-triazol-4-yl) methyl) bambus[6]uril $\underline{15}\left({ }^{1} \mathrm{H}\right.$ NMR $\left.400 \mathrm{MHz}, \mathrm{D}_{2} \mathrm{O}\right)$

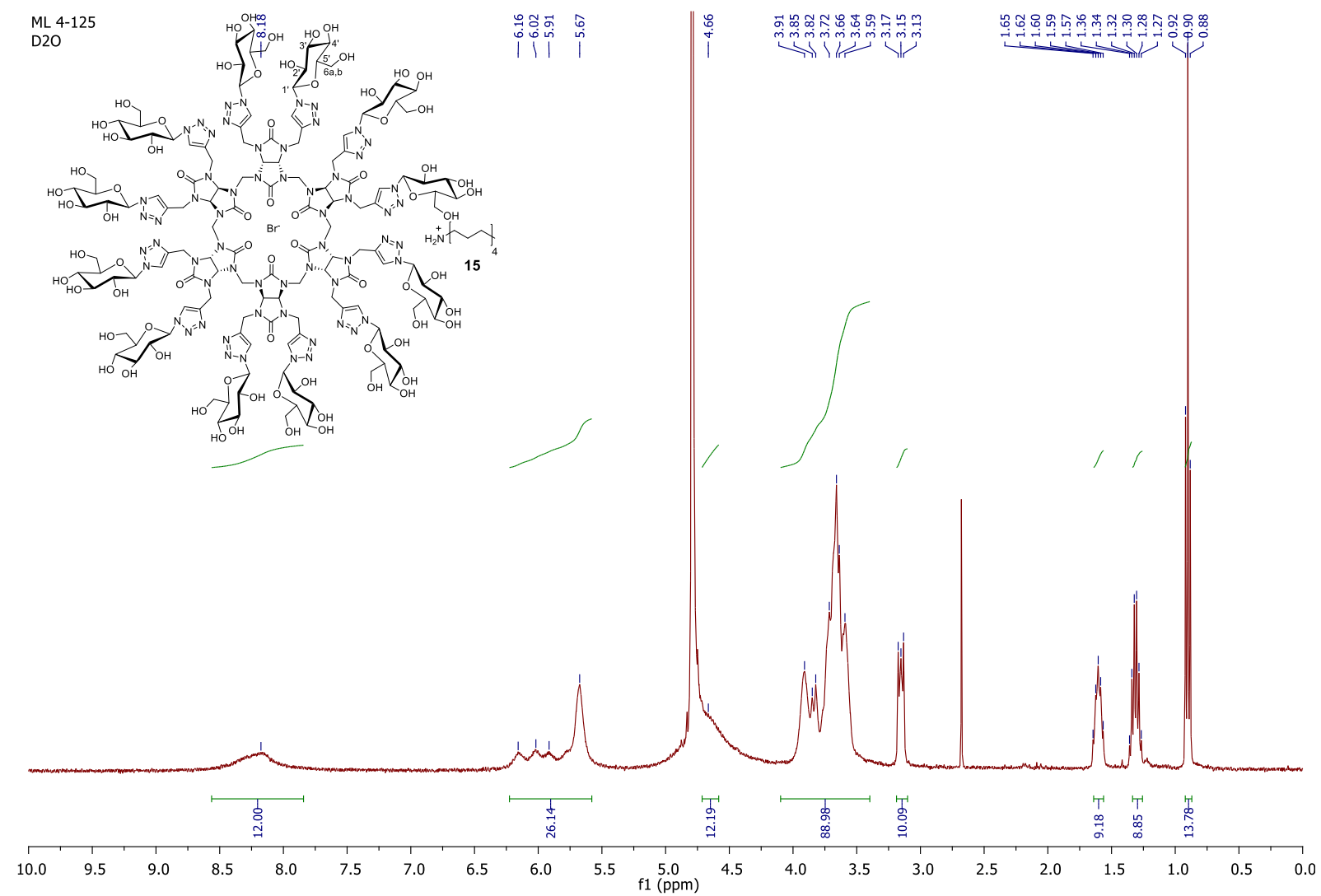




\section{Crystallography Data Collection and Structure Determination}

Crystals of Br@Propargyl ${ }_{12} \mathrm{BU}[6] . T B A 3$ and I@Propargyl ${ }_{12} \mathrm{BU}[6] . T B A 4$ were obtained from $\mathrm{CH}_{2} \mathrm{Cl}_{2} / \mathrm{Et}_{2} \mathrm{O}$ $1 / 1$ solution by slow evaporation of solvent mixture.

The data for compounds 3 and 4.2Et ${ }_{2} \mathrm{O}$ were collected at 150(2) K on a Nonius Kappa-CCD area detector diffractometer ${ }^{6}$ using graphite-monochromated Mo K $\alpha$ radiation $(\lambda=0.71073 \AA$ ). The crystals were introduced into glass capillaries with a protective coating of Paratone- $\mathrm{N}$ oil (Hampton Research). The unit cell parameters were determined from ten frames, then refined on all data. The data (combinations of $\varphi$ - and $\omega$-scans with a minimum redundancy of at least 4 for $90 \%$ of the reflections) were processed with HKL2000. ${ }^{7}$ Absorption effects were corrected empirically with the program SCALEPACK. ${ }^{7}$ The structures were solved by intrinsic phasing with $\mathrm{SHELXT}^{8}$ and refined by full-matrix least-squares on $F^{2}$ with SHELXL-2014. ${ }^{9}$ All non-hydrogen atoms were refined with anisotropic displacement parameters. The hydrogen atoms were introduced at calculated positions and they were treated as riding atoms with an isotropic displacement parameter equal to 1.2 times that of the parent atom (1.5 for $\mathrm{CH}_{3}$, with optimized geometry). In compound 4.2Et $\mathrm{E}_{2} \mathrm{O}$, restraints on some bond lengths, angles and displacement parameters were applied for some atoms in the counter-ion and solvent molecules, which are possibly affected by unresolved disorder. Some voids in the structure of compound $\mathbf{4} \cdot 2 \mathrm{Et}_{2} \mathrm{O}$ likely indicate the presence of other, unresolved solvent molecules. The molecular plots were drawn with ORTEP-3. ${ }^{10}$

Crystal data for 3. $\mathrm{C}_{82} \mathrm{H}_{96} \mathrm{BrN}_{25} \mathrm{O}_{12}, M=1703.74$, monoclinic, space group $C 2 / c, a=25.7303(12), b=$ 13.7910(6), $c=26.5805(10) \AA, \beta=110.176(3)^{\circ}, V=8853.2(7) \AA^{3}, Z=4, D_{c}=1.278 \mathrm{~g} \mathrm{~cm}^{-3}, \mu=0.537$ $\mathrm{mm}^{-1}, F(000)=3576$. Refinement of 545 parameters on 8381 independent reflections out of 116677 measured reflections $\left(R_{\text {int }}=0.024\right)$ led to $R_{1}=0.051, w R_{2}=0.151, S=1.046, \Delta \rho_{\max }=0.33, \Delta \rho_{\min }=-0.64$ e $\AA^{-3}$.

Crystal data for 4-2Et ${ }_{2} \mathrm{O} . \mathrm{C}_{90} \mathrm{H}_{116} \mathrm{IN}_{25} \mathrm{O}_{14}, M=1898.97$, monoclinic, space group $P 2_{1} / n, a=14.3674(4)$, $b=28.2197(15), c=24.7644(13) \AA, \beta=96.004(3)^{\circ}, V=9985.5(8) \AA^{3}, Z=4, D_{c}=1.263 \mathrm{~g} \mathrm{~cm}^{-3}, \mu=0.392$ $\mathrm{mm}^{-1}, F(000)=3984$. Refinement of 1182 parameters on 18939 independent reflections out of 301498 measured reflections $\left(R_{\text {int }}=0.041\right)$ led to $R_{1}=0.067, w R_{2}=0.221, S=1.075, \Delta \rho_{\max }=0.98, \Delta \rho_{\min }=-0.68 \mathrm{e}$ $\AA^{-3}$.
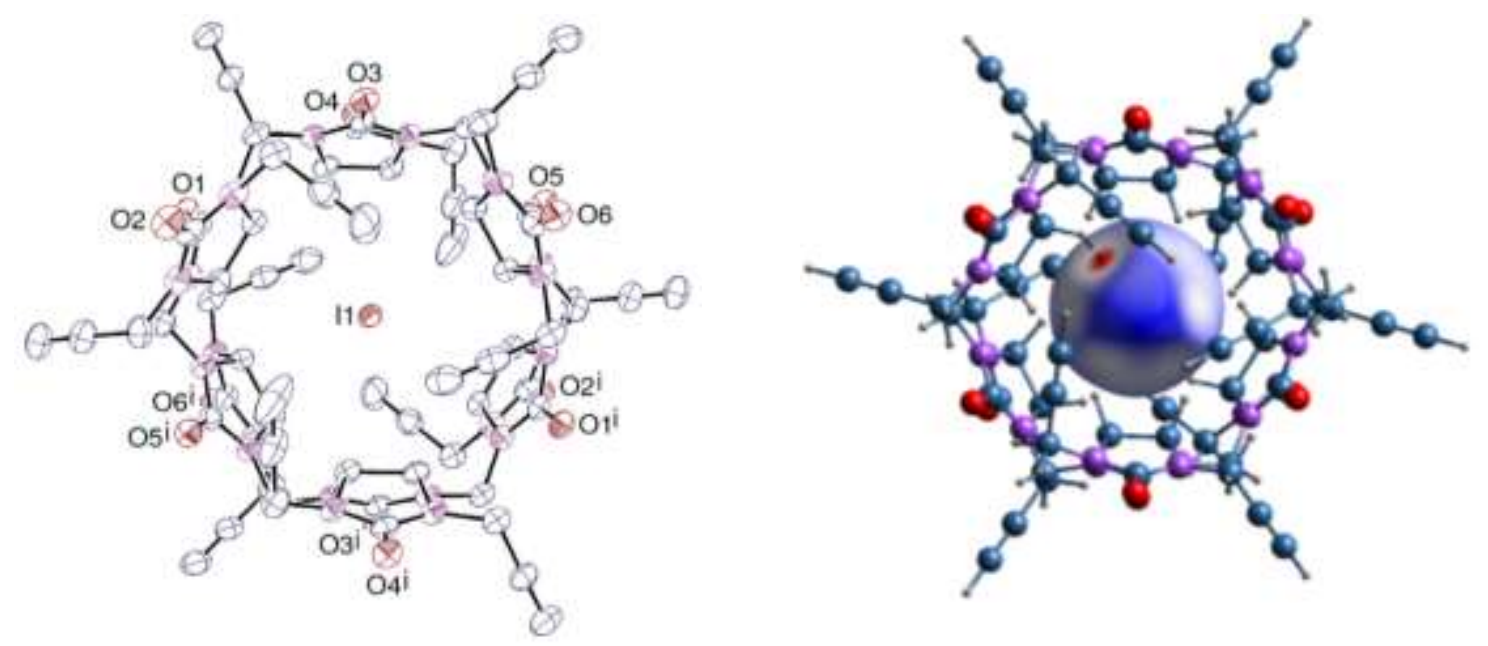

Figure S1. Left : View of one of the two independent molecules in the crystal structure of $4 \cdot 2 \mathrm{Et}_{2} \mathrm{O}$. Displacement ellipsoids are shown at the $30 \%$ probability level. The counterion, solvent molecules and hydrogen atoms are omitted. Symmetry code: $\mathrm{i}=1-x, 1-y, 2-z$. Right : View of the Hirshfeld surface of the iodide anion, showing the $\mathrm{CH}$...halide hydrogen bonding interactions with methine groups of $B U[6]$ (dashed lines). 
Determination of $K_{\mathrm{a}}$ and thermodynamic parameters of complexes by ITC

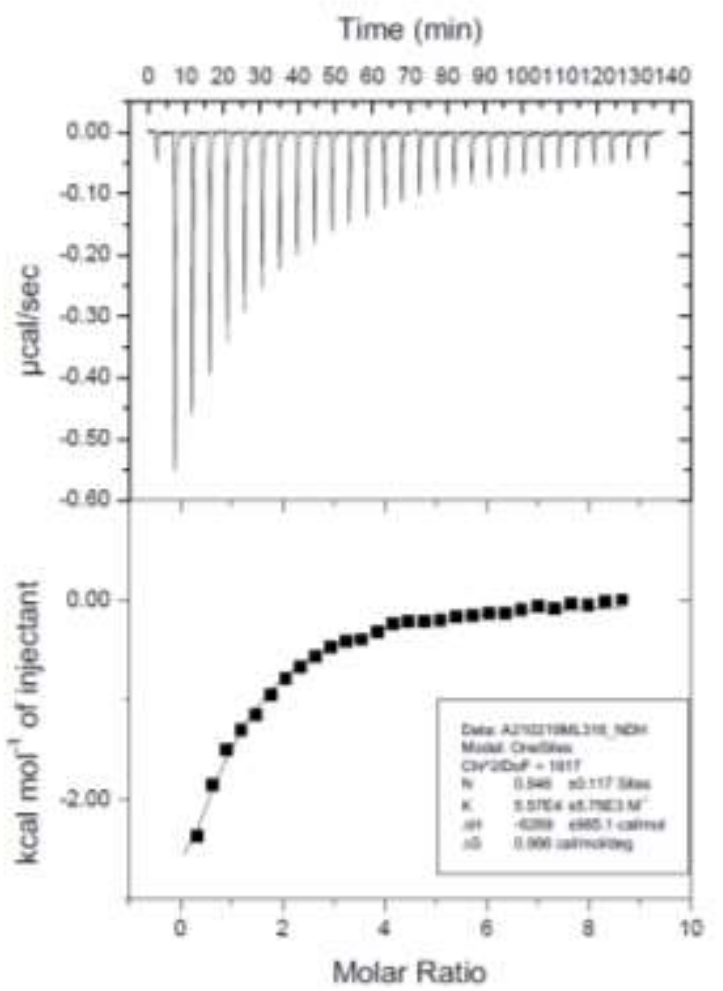

Figure S2 Isothermal titration calorimetry of $\mathrm{I}^{-}$binding to receptor $\mathbf{B U} \mathbf{1 2}\left(\mathrm{R}=\left(\mathrm{CH}_{2}\right)_{3} \mathrm{CO}_{2} \mathrm{H}\right)$ in aqueous phosphate buffer $\left(\mathrm{K}_{2} \mathrm{HPO}_{4} 1.5 \mathrm{mM}\right)$. Top: Data were obtained from the sequential injections of $10 \mu \mathrm{L}$ of guest solution (0.6 mM Nal) to BU $12(0.015 \mathrm{mM})$. Bottom: Plot of the total heat released as a function of the total ligand concentration for the titration shown upper. 


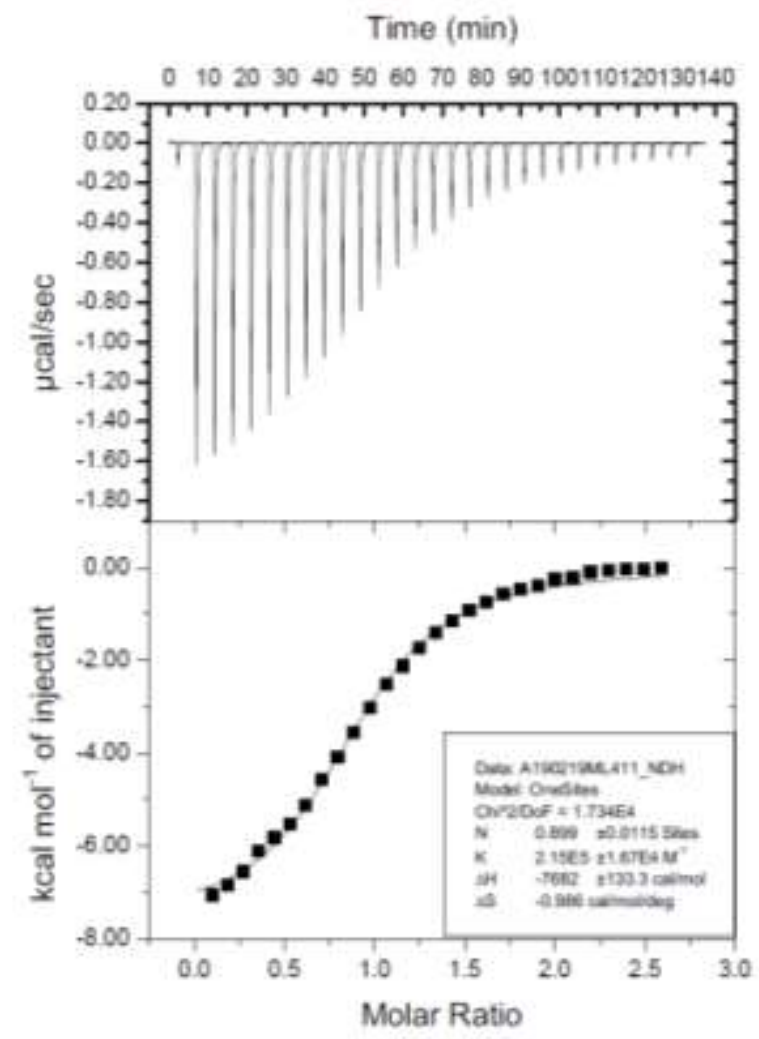

Figure S3. Isothermal titration calorimetry of $\mathrm{I}^{-}$binding to $\mathrm{BU} 13 \mathrm{R}=\mathrm{H}_{\mathrm{HO}}$

Top: Data were obtained from the sequential injections of $10 \mu \mathrm{L}$ of guest solution ( $0.6 \mathrm{mM} \mathrm{Nal})$ to $\mathrm{BU}$ $13(0.05 \mathrm{mM})$. Bottom: Plot of the total heat released as a function of the total ligand concentration for the titration shown upper. 


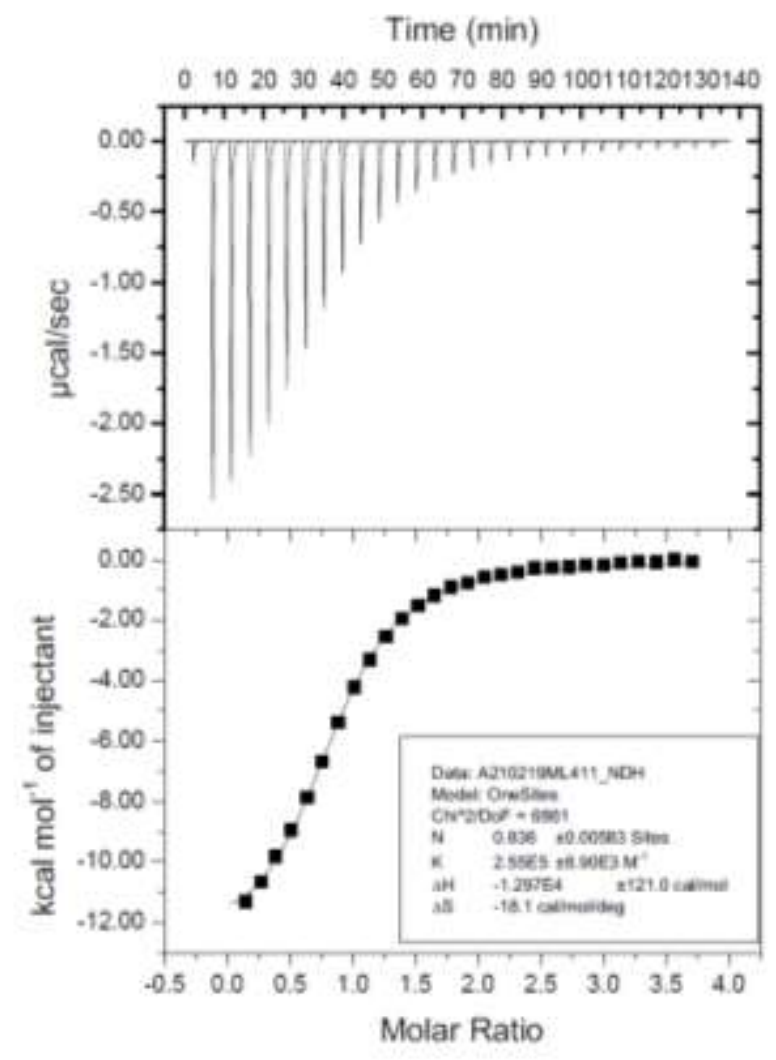

Figure S4. Isothermal titration calorimetry of $\mathrm{I}^{-}$binding to $\mathrm{BU} 13 \mathrm{R}=\mathrm{H}_{\mathrm{Ho}}$ buffer $\left(\mathrm{K}_{2} \mathrm{HPO}_{4} 1.5 \mathrm{mM}\right)$.Top: Data were obtained from the sequential injections of $10 \mu \mathrm{L}$ of guest solution (0.6 mM Nal) to BU $13(0.05 \mathrm{mM})$. Bottom: Plot of the total heat released as a function of the total ligand concentration for the titration shown upper. 


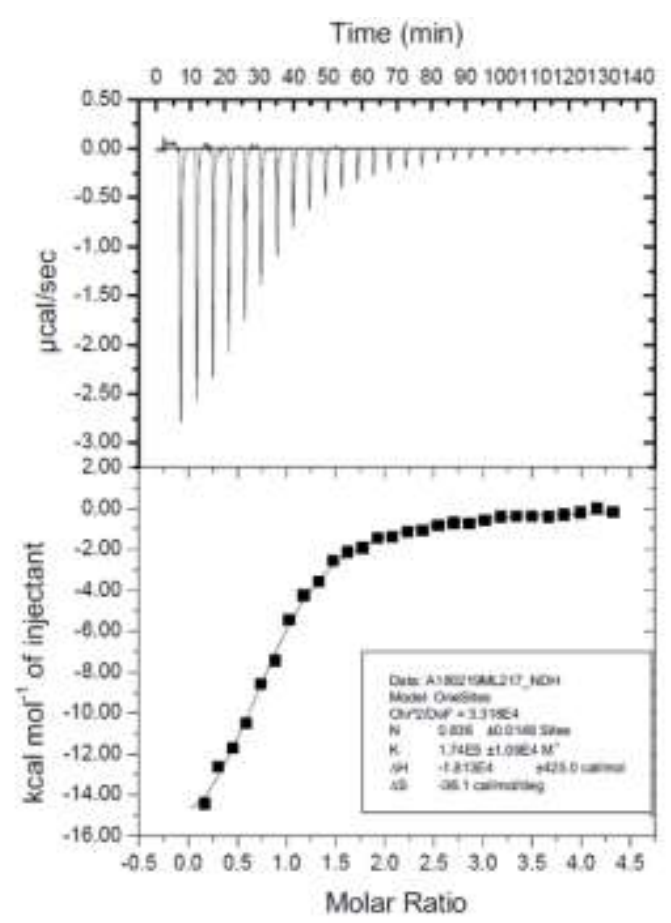

Figure S5. Isothermal titration calorimetry of $\mathrm{I}^{-}$binding to $\mathrm{BU} 14 \mathrm{R}=\mathrm{HO}_{\mathrm{O}}^{\mathrm{HO}} \mathrm{O}_{\mathrm{OH}}$ obtained from the sequential injections of $10 \mu \mathrm{L}$ of guest solution $(0.6 \mathrm{mM} \mathrm{Nal})$ to $\mathbf{B U} 14(0.03 \mathrm{mM})$. Bottom: Plot of the total heat released as a function of the total ligand concentration for the titration shown upper. 


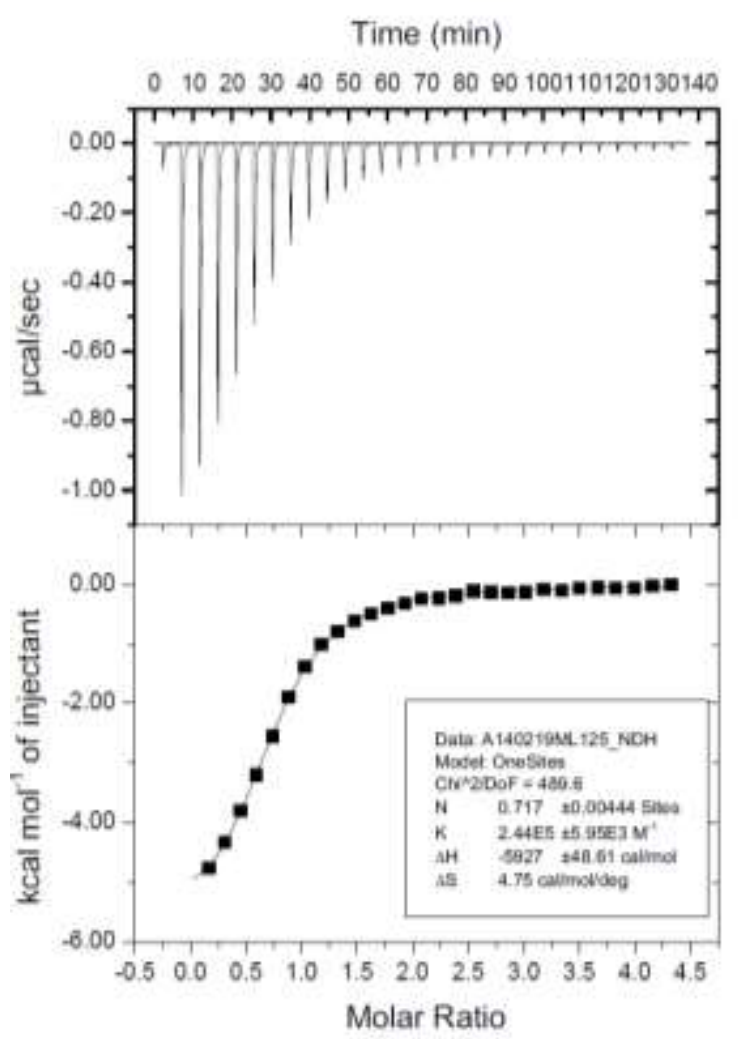

Figure S6 Isothermal titration calorimetry of $\mathrm{I}^{-}$binding to $\mathrm{Br} @ \mathrm{BU} 15 \mathrm{R}=\mathrm{Ho}_{=}^{\mathrm{HO}}$ were obtained from the sequential injections of $10 \mu \mathrm{L}$ of guest solution (0.6 mM Nal) to Br@BU 15 $(0.05 \mathrm{mM})$. Bottom: Plot of the total heat released as a function of the total ligand concentration for the titration shown upper.

\section{References}

[1] J. Svec, M. Dusek, K. Fejfarova, P. Stacko, P. Klán, A. E. Kaifer, W. Li, E. Hudeckova, V. Sindelar, Chem. Eur. J. 2011, 17, 5605.

[2] J. Budhathoki-Uprety, B. M. Novak, Macromolecules 2011, 44, 5947.

[3] B. E. Gryder, M. J. Akbashev, M. K. Rood, E. D. Raftery, W. M. Meyers, P. Dillard, S. Khan, A. K. Oyelere, ACS Chem. Biol., 2013, 8, 2550.

[4] K. Sun, C. Xiao, C. Liu, W. Fu, Z. Wang, Z. Li, Langmuir, 2014, 30, 11040.

[5] S. M. Paterson, J. Clark, K. A. Stubbs, T. V. Chirila, M. V. Baker, J. Polym. Sci. A Polym. Chem., 2011, 49, 4312.

[6] R. W. W. Hooft, COLLECT, Nonius BV, Delft, The Netherlands, 1998.

[7] Z. Otwinowski and W. Minor, Methods Enzymol., 1997, 276, 307.

[8] G. M. Sheldrick, Acta Crystallogr., Sect. A, 2015, 71, 3.

[9] G. M. Sheldrick, Acta Crystallogr., Sect. C, 2015, 71, 3.

[10] L. J. Farrugia, J. Appl. Crystallogr., 2012, 45, 849. 